\title{
HADRONIC DECAYS OF $W$ BOSONS
}

Richard Paul Wilkinson III

A DISSERTATION

in

\section{PHYSICS}

Presented to the Graduate Faculty of the University of Pennsylvania in Partial Fulfillment of the Requirements for the Degree of Doctor of Philosophy

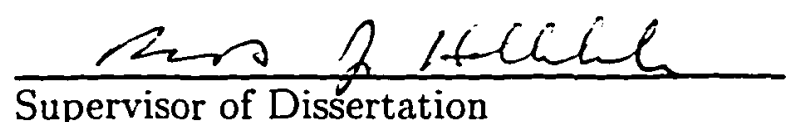

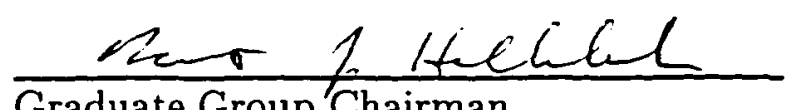

Graduate Group Chairman 
UMI Number: 9800939

UMI Microform 9800939

Copyright 1997, by UMI Company. All rights reserved.

This microform edition is protected against unauthorized copying under Title 17, United States Code. 


\section{ACKNOWLEDGMENTS}

This thesis is dedicated to my family, my parents, Richard and Joanne, and my brother Bill, whose constant love and support made all this possible.

I would like thank my advisor, Professor Bob Hollebeek, for giving me the guidance I needed, the freedom I needed, more computer resources than I could ever need, and, most importantly, the opportunity to work in a world-class research environment while a major discovery in physics was being made.

Also, I would like to acknowledge the contributions and insights of my collaborators at CDF, especially Dr. Brian Harral, Dr. Gary Houk, Prof. Shinhong Kim, Prof. Mark Timko, Prof. Guillaume Unal, Dr. William Wester, and Prof. Brig Williams. Special thanks go to our "godparent" publication review committee, Prof. David Gerdes, Prof. Daniela Bortoletto, and Dr. Mark Kruse, for all their help.

Finally, I would like to thank the many other friends from CDF (Farrukh Azfar, Brendan Bevensee, Wasiq Bokhari, Steve Hahn, Chris Holck, Owen Long, Scott Metzler, Roque Oliveira, Mike \& Merry Starr, and many others) and outside (Andy, Dave, Dex, Steph, et al.) who helped make the difficult times easy and the good times great. 


\section{ABSTRACT \\ HADRONIC DECAYS OF $W$ BOSONS \\ Richard Paul Wilkinson III \\ Robert Hollebeek}

We present evidence for hadronic $W$ decays in $t \bar{t} \rightarrow$ lepton + neutrino $+\geq 4$ jet events using a $109 \mathrm{pb}^{-1}$ data sample of $p \bar{p}$ collisions at $\sqrt{s}=1.8 \mathrm{TeV}$ collected with the Collider Detector at Fermilab ( CDF ). A peak in the dijet invariant mass distribution is obtained in events where two $b$ jets are identified. This peak is consistent with $W$ decay but inconsistent with the predicted background shape by $2.9 \sigma$. We measure the $W$ mass to be $78.3 \pm 5.1$ (stat) \pm 3.0 (syst) $\mathrm{GeV} / \mathrm{c}^{2}$. This result demonstrates the presence of a second $W$ in these $t \bar{t}$ candidates. We show ways in which this result can be used to study $t \bar{t}$ events and detector calibration, for present and future datasets.

We also search for hadronic decay modes from direct $W$ and $Z$ production, but find no stable signal, due to trigger inefficiency. 


\section{Contents}

1 Theory and Motivation 1

1.1 Intermediate Vector Bosons $\ldots \ldots \ldots \ldots$

1.1.1 The $W^{ \pm}$boson $\ldots \ldots \ldots \ldots \ldots$

1.1 .2 The $Z$ boson ..................... 3

1.2 The Top Quark ....................... 3

1.2.1 Semileptonic $t \bar{t}$ decay modes . . . . . . . . . . .

1.3 Motivation to Search for Hadronic Boson Decays . . . . . . . . . . 8

2 Experimental Setup $\quad 10$

2.1 The Tevatron Accelerator .................... 11

2.2 Tracking Chambers . . . . . . . . . . . . . . 13

2.2.1 Central Tracking Chamber (CTC) . . . . . . . . 14

2.2.2 Vertex Time Projection Chamber (VTX) ........ 15

2.2 .3 Silicon Vertex Detector $(\mathrm{SVX}) \ldots \ldots \ldots \ldots$

2.3 Calorimetry .............................. 17

2.3.1 Central Calorimetry (CEM, CHA, WHA) . . . . . 18

2.3.2 Plug Calorimetry (PEM, PHA) ............ 19

iv 


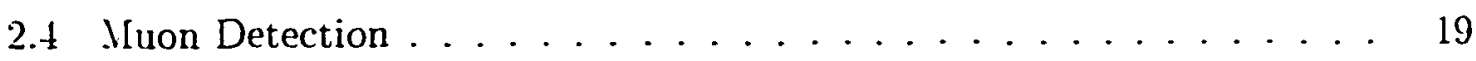

2.4 .1 Central Muon Chamber (CMU) ............ 20

2.4.2 Central Muon Upgrade (C.MP) . . . . . . . . 20

2.4 .3 Central Muon Extension (CMX) ............ 20

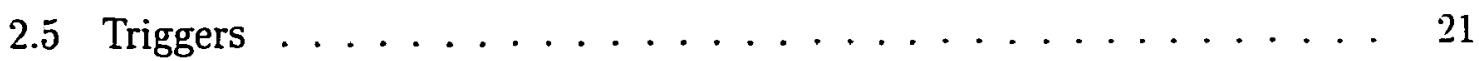

2.5 .1 Beam-Beam Counters (BBC's) . . . . . . . . . . 21

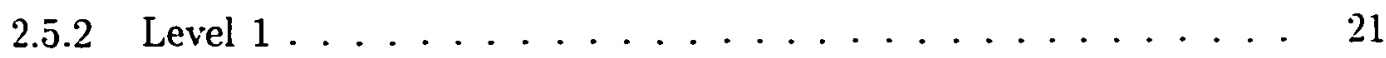

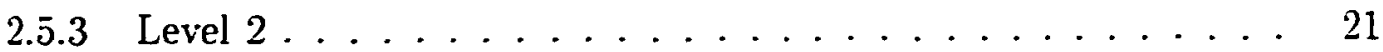

2.5 .4 Level $3 \ldots \ldots \ldots \ldots \ldots \ldots \ldots . \ldots \ldots \ldots$

2.6 Offline Reconstruction . . . . . . . . . . . . 22

3 Event Reconstruction $\quad 24$

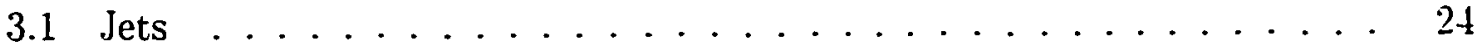

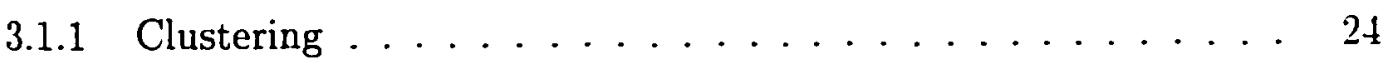

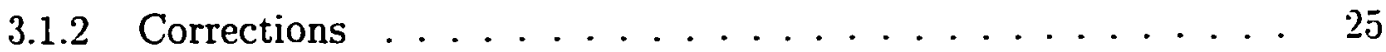

3.2 High $P_{T}$ Lepton Selection $\ldots \ldots \ldots \ldots . \ldots . \ldots . \ldots$

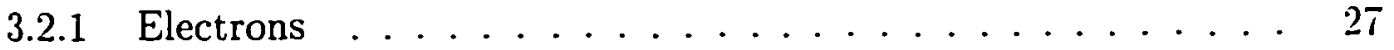

3.2 .2 Muons ............................. 29

3.3 . Vissing $E_{T} \ldots \ldots \ldots \ldots \ldots \ldots \ldots \ldots \ldots$

3.3.1 Missing $E_{T}$ trigger $\ldots \ldots \ldots \ldots \ldots \ldots \ldots$

3.4 Heavy Flavor Tagging . . . . . . . . . . . . . . . . 32

3.4.1 Secondary Vertex Tagging .............. 32 
3.4 .2 Soft Lepton Tagging ...................... 34

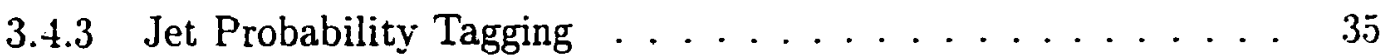

3.4.4 Comparison of tagging efficiencies ........... 38

4 Hadronic $W$ decays in $t \bar{t}$ events $\quad 39$

4.1 Event Sample .......................... 39

4.2 Monte Carlo Simulation ... . . . . . . . . . . . . 40

4.3 Technique ......................... 40

4.3 .1 Dijet masses ...................... 40

$4.3 .2 \quad b$ tagging.......................... 41

4.3 .3 Loose $b$ tagging . . . . . . . . . . . . . 42

4.3 .4 Triple-tagged events . . . . . . . . . . . . . 43

4.4 Monte Carlo Simulation Studies . . . . . . . . . . . . 45

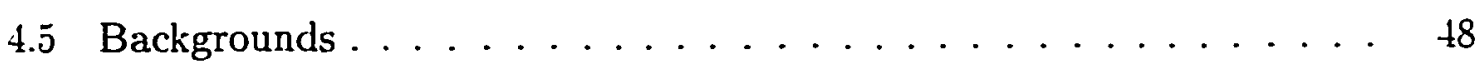

4.5 .1 Fake tag rates ..................... 49

4.5 .2 Single top backgrounds . . . . . . . . . . . . 49

4.5 .3 Non- $W$ background $\ldots \ldots \ldots \ldots . \ldots . \ldots . \ldots . \ldots$

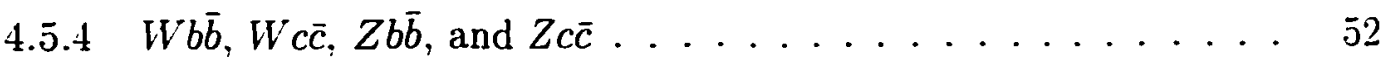

4.5.j Final background estimate ............. 52

4.5 .6 Background shape $\ldots \ldots \ldots \ldots \ldots \ldots \ldots$

4.6 Results ............................. 54

4.7 Notable Events . . . . . . . . . . . . . . 55

vi 
4.8 Hadronic $W$. Mass Fitting . . . . . . . . . . . 62

4.8.1 Background term ................... 62

4.8.2 Constraining $N_{W}$ and $N_{c} \ldots \ldots 63$

4.8.3 Comparison of likelihood functions .......... 6 T

4.8 .4 Systematic uncertainties . . . . . . . . . . . 69

4.9 Significance of the Result . . . . . . . . . . . 78

4.9.1 Mass Window Technique .............. 78

4.9 .2 Likelihood Technique .............. 78

$4.10 t \bar{t}$ Event Reconstruction .................... 79

4.10 .1 Introduction $\ldots \ldots \ldots \ldots \ldots \ldots \ldots$

4.10 .2 Kinematic fitting of the top mass . . . . . . . 80

4.10 .3 Three-body balancing technique ............ 81

4.10 .4 Comparison to fitting technique . . . . . . . . 82

4.10.5 Mass-balancing results from data .......... 87

4.10 .6 Systematic uncertainties . . . . . . . . . . . 9 90

4.10 .7 Results . . . . . . . . . . . . . . . . . 92

4.11 Measurement of the Jet Energy Scale . . . . . . . . . . . 92

4.11 .1 Introduction . . . . . . . . . . . . . . 92

4.11 .2 Preliminary measurement . . . . . . . . . . 94

4.11 .3 Likelihood method . . . . . . . . . . . . 95

4.11 .4 Systematic uncertainties . . . . . . . . . . 96

4.11 .5 Results ........................... 96

vii 
4.11 .6 Future precision .................... 9 T.

4.12 Determining the Hard Gluon Radiation Content . . . . . . . . 9 9 i

4.12 .1 Preliminary look ... . . . . . . . . . . 98

4.12 .2 Likelihood fit .................. 101

4.12 .3 Systematic uncertainties . . . . . . . . . . . 103

4.12 .4 Results ........................ 103

4.12 .5 Future precision ..........................

5 Single $W$ production $\quad 106$

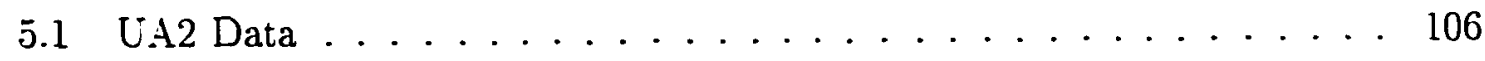

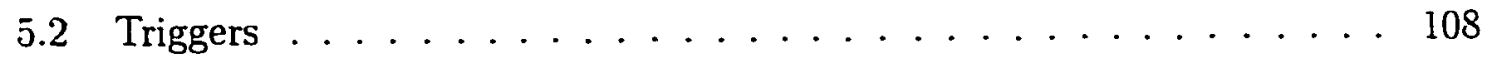

5.3 Selection Criteria . . . . . . . . . . . . . . 108

$5.3 .1 W / Z$ signal expectation $\ldots \ldots \ldots \ldots \ldots$

5.4 Measuring the Dijet Trigger Efficiency ........... 111

j.4.1 Monte Carlo test of the method ............ 112

5.4 .2 Comparing efficiencies to dijet data .......... 112

5.4 .3 . Wultiple interactions ........................ 115

5.4 .4 Eta dependence ............................ 115

5.4.j Final trigger efficiency curves ............. 118

5.4 .6 Correcting for jet $E_{T}$ cuts ............... 119

5.5 Analysis of Dijet Data ..................... 120

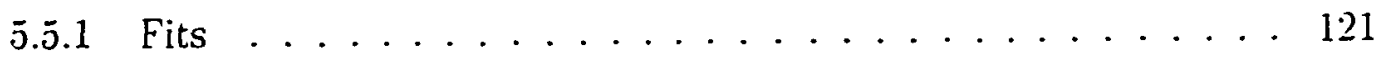

viii 
5.6 Toy Monte Carlo studies . . . . . . . . . . . . . . 121

$5.7 \quad b$ tagging results $\ldots \ldots \ldots \ldots \ldots \ldots \ldots \ldots \ldots$

5.8 Future trigger requirements $\ldots \ldots \ldots \ldots \ldots \ldots . \ldots \ldots$

$6 W^{+} W^{-}$production 133

$\begin{array}{llr}7 \text { Conclusion } & 136\end{array}$

$\begin{array}{ll}\text { A The CDF Collaboration } & 138\end{array}$

References ................................. 142 


\section{List of Tables}

1.1 Decay modes of the $W$ boson $\ldots \ldots \ldots \ldots . \ldots \ldots$

1.2 Decay modes of the $Z^{0}$ boson, from Ref. [5]. . . . . . . . . . . . 4

1.3 Decay modes in $t \bar{t}$ events . . . . . . . . . . . . . . 6

1.4 NLO calculations of the $t \bar{t}$ cross section, including gluon resummation. $\quad \bar{\imath}$

2.1 Energy resolutions of the various calorimeter components. . . . . 17

3.1 Central electron trigger requirements . . . . . . . . . . . 28

3.2 Tag rates for $b$ and $c$ jets, and mistag rates for the three taggers. . . 38

4.1 The probabilities that the various forms of triple-tagged events correctly isolate the hadronic $W$ signal. . . . . . . . . . . . ti

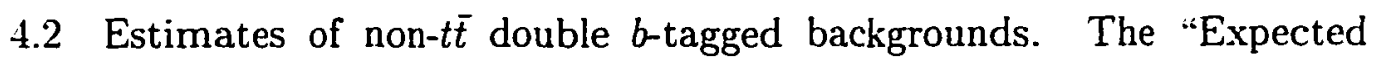
backgrounds" are the top mass analysis estimates, requiring only one tag, and the "Excess backgrounds" are obtained by subtracting the heavy-flavor-pair backgrounds from the total expected background.

4.3 Double-tagged events and their hadronic $W$ mass and the transverse masses of the leptonic $W$ candidates. . . . . . . . . . 59

4.4 Double-tagging rates for events with and without one or more gluon jets. 65 
4.5 Systematic uncertainties on the $W^{-}$mass measurement due to fit tech-

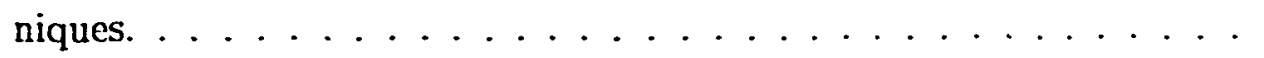

4.6 Systematic uncertainties on the $W$ mass measurement due to different background constraints. ..............

4.7 Systematic uncertainty on the $W$ mass measurement due to uncertainties in the jet energy scale. ...............

4.8 Summary of the systematic uncertainties on the measurement of the hadronic $W$ mass. . . . . . . . . . . . . . $\ldots$ it

4.9 Results of the top mass measurement using the three-body mass balancing technique. $\ldots \ldots \ldots \ldots \ldots$

4.10 Systematic uncertainty on the top mass due to uncertainty on the amount of hard gluon radiation. . . . . . . . . . . 91

4.11 Systematic uncertainty on the top mass due to fit technique. . . . . 92

4.12 Systematic uncertainty on the top mass result from the three-body balancing technique. .................... 93

4.13 Systematic uncertainties on the measurement of the jet energy scale. . 96

4.14 Expected future precision of the jet energy scale measurement. . . . . $9 \vec{\imath}$

4.15 The fraction of pseudo-experiments with eight (or more) events in the mass window $60-100 \mathrm{GeV} / c^{2}$, as a function of the fraction of input events with one or more gluon jets. . . . . . . . . . . 99

4.16 Systematic uncertainties to the measurement of the gluon fraction in $t \bar{t}$ events. . . . . . . . . . . . . . . . . 104 $\mathrm{xi}$ 
4.1T Expected statistical precision of future measurements of the gluon fraction. ........................... 10.5

5.1 Efficiencies of kinematic cuts. . . . . . . . . . . 110

$5.2 \mathrm{~W}$ and $\mathrm{Z}$ cross-sections after cuts. . ................ 111

5.3 Magnitudes of the fake bumps produces by varying the trigger efficiency corrections. The expected signal is about 4000 events. . . . . . 122

5.4 Results of ensembles of 500 dijet pseudo-experiments. The input mass was $80.33 \mathrm{GeV} / c^{2} \ldots \ldots \ldots \ldots \ldots \ldots \ldots \ldots$ 


\section{List of Figures}

1.1 Top quark pair production through a) quark-antiquark annihilation, and b) gluon-gluon processes. The cross section for the first process is five times larger than the other three combined, at Tevatron energies. $\quad 5$

1.2 An example of $W+$ multijet production. . . . . . . . 8

2.1 A cross section of the CDF detector. . . . . . . . . 11

2.2 A schematic of the accelerator complex. The CDF detector is located

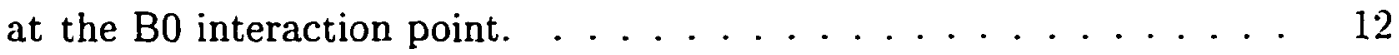

2.3 An end view of the Central Tracking Chamber. ......... 14

2.4 One barrel of the SVX. . . . . . . . . . . . 16

2.5 The tower segmentation of the CDF calorimetry. . . . . . . 18

3.1 Top-specific correction factors to the Jet $E_{T} \ldots \ldots{ }_{2}$ i

3.2 Simplified view in the $x y$ plane of SVX tracks forming a displaced vertex. 33

3.3 The distribution of jet probability for jets in the inclusive jet sample, and for jets near muon candidates. Low jet probability means the tracks are not consistent with the primary vertex. . . . . . 3 i

4.1 Dijet invariant mass from hadronic $W$ decay in $t \bar{t}$ Monte Carlo. . . . 42 xiii 
4.2 Jet probability of jets not tagged by SECLTX or SLT in $H^{\circ}+\geq 3$ jet

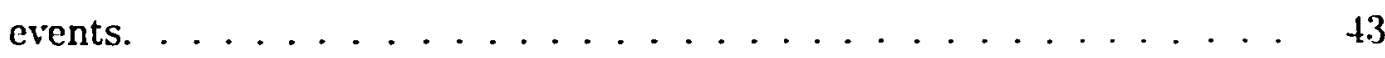

4.3 Jet probability of jets not tagged by SECVTX or SLT in $t \bar{t}$ :Monte Carlo. 44

4.4 Number of tracks passing SECVTX pass 1 cuts with an impact parameter significance of three or more in $b$ jets tagged by SECVTX and

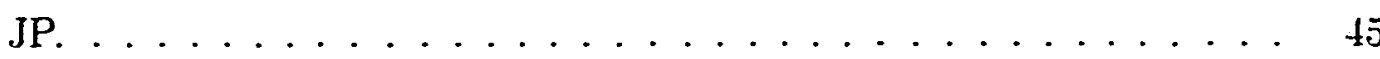

4.5 Dijet mass spectrum for the untagged jets in Monte Carlo for double b-tagged events. The shaded region corresponds to generated hadronic $W$ decay. . . . . . . . . . . . . . . . . . 46

4.6 Single top quark production through a) virtual $W$ and b) $W$-gluon fusion. 50

4.7 Comparison of the background histogram shapes with $Q^{2}=M_{W}^{2}$ before and after weighting entries by mistag probabilities. . . . . . . 54

4.8 Dijet mass spectrum after demanding at least two $b$ tags, one of which must be SECVTX or SLT. . . . . . . . . . . . 56

4.9 Comparison of dijet masses excluding loose $b$-tags with the other five combinations in the event which involve $b$-tagged jets, and the pairs of $b$-tagged jets. All jets are corrected as $W$ jets. . . . . . . .

4.10 The lepton-neutrino transverse mass for the eight events in the hadronic $W$ mass window $60-100 \mathrm{GeV} / c^{2}$, along with Monte Carlo expectation for $t \bar{t}$ events. ......................... 58

4.11 The low dijet-mass event: Run 66573 , Event 107219. . . . . . . . 60

4.12 The high dijet-mass event: Run 43351, Event 266423. . . . . . . . 61 xiv 
4.13 Background shapes used for a) non-t $\bar{t}$ background and b) top combinatorics

4.14 The relation between gluon fraction and $W$ purity for a double-tagged

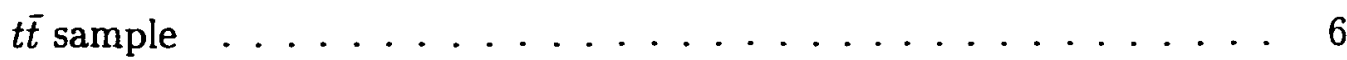

4.15 Comparison of the smeared and unsmeared binomial constraints on $N_{W}$. 67

4.16 Pull distributions from 1000 pseudo-experiments of 11 events each. 68

4.17 Distributions from 1000 pseudo-experiments of 11 events each. Arrows represent the value obtained fitting to the data. . . . . . . 70

4.18 Comparison of the background histograms with $Q^{2}=M_{W}^{2}$ and $Q^{2}=P_{T}^{2}$. 71

4.19 The change in likelihood with and without a fixed Gaussian signal term, for simulated experiments which use only background events. The line indicates the value of 4.35 from the data. . . . . . . 80

4.20 The mass difference between the leptonic and hadronic sides of the reconstructed top decay, from $t \bar{t}$. Wonte Carlo. . . . . . . . . .

4.21 The reconstructed top mass for $175 \mathrm{GeV} / c^{2}$. Monte Carlo. The dotted line is the shape of events in the correct configuration.

4.22 Wean reconstructed top mass, obtained by fitting to a Gaussian, for the mass-balancing and fitting techniques. . . . . . . . . 85

4.23 The Gaussian widths of the top mass distributions for the two techniques. 86

4.24 The distribution of pulls of the top mass, from pseudo-experiments. 88 $\mathrm{xv}$ 
4.25 The top masses obtained from these seven events. A likelihood fit to Wonte Carlo templates of different top masses yields a measured top mass of $182.3 \pm 9.6 \mathrm{GeV} / c^{2} \ldots \ldots \ldots \ldots \ldots$

4.26 The relation between jet energy scale and median returned $W$ mass from ensembles of pseudo-experiments. The large point represents the measurement in the data, which translates into a rough estimate of the jet energy scale of $(-2.5 \pm 8.8) \%$.

4.27 The results of a likelihood fit to templates with various jet energy scale shifts.

4.28 The number of events in a mass window of $60-100 \mathrm{GeV} / c^{2}$ for 10000 pseudo-experiments with gluon fractions of $25 \%, 55 \%$, and $85 \% . \ldots 100$

4.29 Templates with various mixes of gluon and non-gluon $t \bar{t}$ events. . . 101

4.30 Median returned gluon fraction from pseudo-experiments using differ-

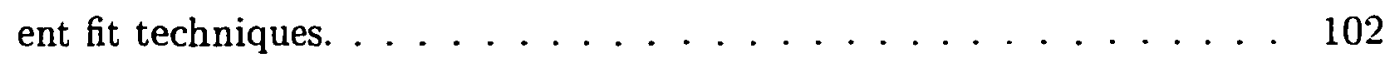

4.31 Results of the likelihood fit to the data. . . . . . . . . 103

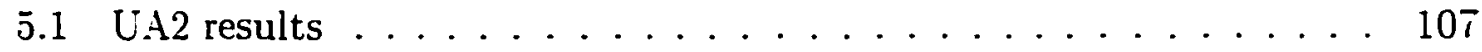

5.2 Comparing the $12 \mathrm{GeV}$ trigger efficiencies for JET_20 samples in runs

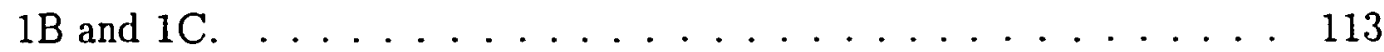

5.3 The .Monte Carlo simulated trigger efficiencies for a jet to have a trigger $E_{T}$ of $12 \mathrm{GeV}$ or greater, for jets selected by both inclusive jet and dijet criteria.

xvi 
5.4 The top plot compares the efficiency for jets to have a trigger $E_{T}$ greater than $12 \mathrm{GeV}$, given that the trigger $E_{T}$ is already greater than $10 \mathrm{GeV}$, for DIJET_10 and inclusive jet data. The bottom plot compares the efficiency for having $15 \mathrm{GeV}$ given $12 \mathrm{GeV}$, using the DIJET_12 and inclusive jet samples. ................ 116

5.5 The dependence of the efficiency curves on the number of event vertices in the event. ...................... 117

5.6 The dependence of the efficiency curves on jet pseudorapidity. . . . . 118

5.7 Trigger efficiency, as a function of raw jet $E_{T}$, for the bins in number of vertices and pseudorapidity. The three $\eta$ bins correspond to $0.1<$ $|\eta|<0.5,0.5<|\eta|<0.7$, and $0.7<|\eta|<0.9 \ldots \ldots \ldots \ldots$

5.8 The efficiency of the raw jet $E_{T}$ thresholds of 12 and $15 \mathrm{GeV}$, as a function of corrected dijet mass. A correction is applied to the dijet mass spectrum, as a function of the raw dijet masses. . . . . . . . 126

5.9 The top plot shows the dijet mass spectrum for DIJET_12 triggers. with and without trigger efficiency corrections. The bottom plot is the trigger efficiency. .................. 12

5.10 The difference between the data and fitted background. No signal is seen

xvii 
5.11 Applying a trigger efficiency that is incorrect by a small amount leads to a fake bump in a smooth dijet mass spectrum. The variation comes from shifting the single-jet trigger efficiency by the amount of the discrepancy in Fig. 5.4a. . . . . . . . . . . . . . 129

5.12 The SECVTX tagged data and fake rates. The bottom plot divides the data by the fake rate. All entries are weighted by their DIJET_12 trigger efficiency. ..................... 130

5.13 The top plot shows the corrected dijet mass spectrum for events with two SECVTX tags. The bottom shows events with one SECVTX tag, and one tag of either SECVTX, SLT, or Jet Probability $<5 \% \ldots . .131$

5.14 Trigger efficiency for a Level 2 trigger with $E_{T}>8 \mathrm{GeV},|\eta|<0.8$, and $\Sigma E_{T}>25 \mathrm{GeV}$, obtained from the low $P_{T}$ muon sample. . . . . 132

6.1 The dijet mass spectrum for the CDF $W+2$ jet sample, with the expected $W W$ and $W Z$ contribution superimposed. 


\section{Chapter 1}

\section{Theory and Motivation}

\subsection{Intermediate Vector Bosons}

A central tenet of the quantum theory of particle interactions is that forces are mediated by particles, quanta of the force's field. This work focuses on a mediator of the weak nuclear force, the $W$ boson.

In the Standard Model of electroweak interactions [1, 2], the differences between the electromagnetic and weak interactions result from spontaneous symmetry breaking, via the Higgs mechanism. A consequence of this theory is that the four gauge bosons associated with the $S U(2)_{L} \otimes U(1)_{Y}$ symmetry of the electroweak Lagrangian manifest themselves as one massless electromagnetic boson (the photon) and three massive weak bosons (the $W^{+}, W^{-}$, and $Z^{0}$ ).

\subsubsection{The $W^{ \pm}$boson}

The $W$ charged intermediate vector boson was experimentally discovered in 1983 at the CERN $p \bar{p}$ collider $[3,4]$. Current measurements [5] place the $W$ mass at $80.33 \pm$ $0.15 \mathrm{GeV} / \mathrm{c}^{2}$. 
The $W^{*}$ was discovered in the decay mode $W^{ \pm} \rightarrow e^{ \pm} \nu_{e}$. The decay width for this process is now known to be $\Gamma_{e}=(2.07 \pm 0.06) \mathrm{GeV}$. The leptonic decay modes $W^{ \pm} \rightarrow \mu^{ \pm} \nu_{\mu}$ and $W^{ \pm} \rightarrow \tau^{ \pm} \nu_{\tau}$ have also been observed, with very similar widths, as expected, since the $W$ mass is much larger than any lepton mass.

The $W$ is also expected to decay into quark pairs, with the same coupling strength as the leptonic decays. The decay rates for quark processes are enhanced by a factor of three because of the three quark colors, and affected by the relevant CKM matrix element [6]:

$$
\Gamma\left(W \rightarrow q q^{\prime}\right)=3\left|V_{q q^{\prime}}\right|^{2} \Gamma_{e}
$$

From this, we expect $W$ 's to decay leptonically $1 / 3$ of the time, and hadronically $2 / 3$ of the time. Ignoring quark masses and off-diagonal CKM elements, half of the hadronic decays will be of the form $W \rightarrow u \bar{d}$, and half will be of the form $W \rightarrow c \bar{s}$. The $W$ decay modes are summarized in table 1.1 .

The electron and muon decay modes are cleanly identified by the presence of an energetic lepton and a momentum imbalance due to the undetected neutrino. Hereafter, we refer to datasets of leptonically-decaying $W$ candidates simply as $I^{-}$ samples.

The hadronic modes are much more difficult to isolate. The strong force has the property that free quarks and gluons are not seen in nature; instead they hadronize, creating quark-antiquark pairs from the vacuum. The quarks from $W$ decay will be seen as jets, collimated showers of mesons and baryons. Thus, the experimental 


\begin{tabular}{||c|c||}
\hline Wode & Fraction $\left(\Gamma_{i} / \Gamma\right)$ \\
\hline$W \rightarrow e \overline{\bar{e}}$ & $1 / 9$ \\
$W \rightarrow \mu \overline{\nu_{\mu}}$ & $1 / 9$ \\
$W \rightarrow \tau \overline{\nu_{\tau}}$ & $1 / 9$ \\
\hline$W \rightarrow u \bar{d}$ & $1 / 3$ \\
$W \rightarrow c \bar{s}$ & $1 / 3$ \\
\hline
\end{tabular}

Table 1.1: Decay modes of the $W$ boson

signature for hadronic $W$ decay is two jets with an invariant consistent with the $W$ mass.

\subsubsection{The $Z$ boson}

The $Z^{0}$ neutral vector boson was also discovered in 1983 at CERN $[\pi, 8]$. The $Z$ has been extensively studied at $e^{+} e^{-}$colliders at CERN and SLAC, and its mass is currently known [5] to be $(91.187 \pm 0.007) \mathrm{GeV} / c^{2}$. The decay modes of the $Z$ are given in table 1.2.

\subsection{The Top Quark}

The top quark is the isospin partner of the bottom quark. Although it has been expected ever since the discovery of the bottom quark in 1977 [9], it was not directly observed until 1995, by the CDF and D0 collaborations at Fermilab's Tevatron accelerator $[10,11]$. The reason for the difficulty in finding the top quark is its high 


\begin{tabular}{|c|c||}
\hline Mode & Fraction $\left(\Gamma_{i} / \Gamma\right)$ \\
\hline$e^{+} e^{-}$ & $(3.366 \pm 0.008) \%$ \\
$\mu^{+} \mu^{-}$ & $(3.367 \pm 0.013) \%$ \\
$\tau^{+} \tau^{-}$ & $(3.360 \pm 0.015) \%$ \\
$\nu \bar{\nu}$ & $(20.01 \pm 0.16) \%$ \\
$b \bar{b}$ & $(15.46 \pm 0.14) \%$ \\
hadrons & $(69.90 \pm 0.9) \%$ \\
\hline
\end{tabular}

Table 1.2: Decay modes of the $Z^{0}$ boson, from Ref. [5].

mass; CDF measures the top mass [12] to be $175.6 \pm 4.4($ stat $) \pm 4.8($ syst $) \mathrm{GeV} / c^{2}$. and D0 [13] measures $173.3 \pm 5.6($ stat $) \pm 6.2($ syst $) \mathrm{GeV} / c^{2}$. The top quark is much heavier than any other known quark, and its large mass implies a special relationship to the Higgs mechanism, which generates particle masses in the Standard Model.

The dominant production mechanism for top quarks at the Tevatron is the creation of top-antitop pairs from quark-antiquark annihilation, shown in figure 1.1(a). Also contributing are the gluon fusion mechanisms of figure 1.1(b), though with a combined effect smaller by a factor of five. The $q \vec{q}$ processes dominate because quarks are more likely than gluons to carry a large fraction of the proton or antiproton momentum.

Each top quark is expected to immediately decay, nearly always into a $b$ quark and an on-shell $W$ boson. The $W$ 's then decay as detailed in section 1.1.1. Independent $t$ and $\bar{t}$ decays give the topologies for a $t \bar{t}$ event shown in table 1.3 . 

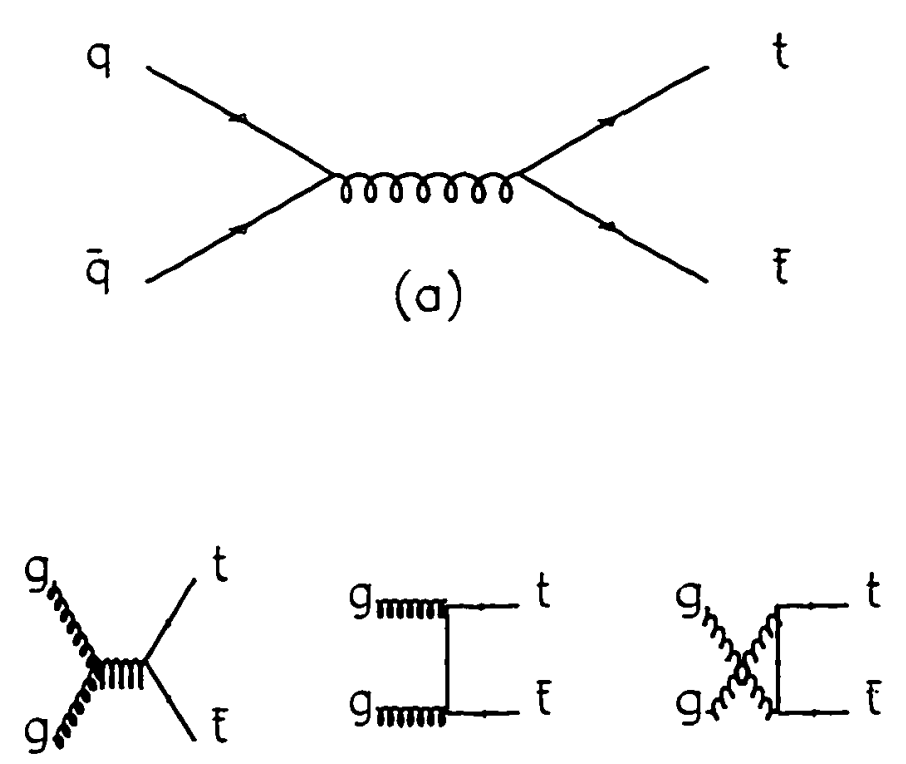

(b)

Figure 1.1: Top quark pair production through a) quark-antiquark annihilation, and b) gluon-gluon processes. The cross section for the first process is five times larger than the other three combined, at Tevatron energies.

The first set of decay modes in table 1.3 are referred to as dileptonic modes, the second semileptonic modes, the third all-hadronic modes, and the fourth tau modes. The dilepton modes provide the cleanest signal, but their usefulness is limited by their low branching fractions and the presence of two neutrinos. All-hadronic modes, on the other hand, have large branching ratios, but are difficult to distinguish from a large background of $\mathrm{QCD}$ multijet events. The semileptonic modes have been most useful so far in studying the top quark, and in Chapter 4 we will present evidence for 


\begin{tabular}{||c|c||}
\hline Process & Branching Ratio \\
\hline$e \nu b e \nu b$ & $1 / 81$ \\
$e \nu b \mu \nu b$ & $2 / 81$ \\
$\mu \nu b \mu \nu b$ & $1 / 81$ \\
\hline$e \nu b j j b$ & $12 / 81$ \\
$\mu \nu b j j b$ & $12 / 81$ \\
\hline$j j b j j b$ & $36 / 81$ \\
\hline$\tau \nu b \mathrm{Xb}$ & $17 / 81$ \\
\hline
\end{tabular}

Table 1.3: Decay modes in $t \bar{t}$ events

hadronic $W$ decays in these events.

The top quarks have a lifetime of $\approx 5 \times 10^{-25} \mathrm{~s}$, which is smaller than the characteristic scale of QCD hadronization processes, $\mathrm{O}\left(10^{-23} \mathrm{~s}\right)$ [14]. Top quarks therefore decay before they have a chance to hadronize, so they decay as free quarks, and their decay products follow an angular distribution for spin-1/2 particles.

CDF measures the cross section for producing $t \bar{t}$ pairs of mass $175 \mathrm{GeV} / c^{2}$ to be $7.7_{-1.5}^{+1.8} \mathrm{pb}[15]$, while D0 measures $5.77 \pm 1.76 \mathrm{pb}$ at $m_{t}=170 \mathrm{GeV} / \mathrm{c}^{2}[16]$. Nextto-leading-order, $\mathrm{O}\left(\alpha_{s}^{3}\right)$, predictions of the cross section are shown in table 1.4. Sote that these cross sections are ten orders of magnitude lower than the total inelastic $p \bar{p}$ cross section. 


\begin{tabular}{||c|c||}
\hline Calculation & $\sigma(t \bar{t})$ \\
\hline Laenen et al. $[1 \overline{7}]$ & $4.94_{-0.54}^{+0.28} \mathrm{pb}$ \\
Berger et al. $[18]$ & $5.52_{-0.45}^{+0.07} \mathrm{pb}$ \\
Catani et al. $[19]$ & $4.75_{-0.68}^{+0.63} \mathrm{pb}$ \\
\hline
\end{tabular}

Table 1.4: NLO calculations of the $t \bar{t}$ cross section, including gluon resummation.

\subsubsection{Semileptonic $t \bar{t}$ decay modes}

The analyses in Chapter 4 will focus on the semileptonic decay modes. These modes. combined with $b$ quark identification discussed in section 3.4 , were the most powerful for Fermilab's discovery and top mass measurement, and are expected to be the best modes for further studies of $t \bar{t}$ event kinematics in the future.

Events resulting from these decay modes are characterized by an energetic electron or muon, four or more jets from the hadronized quarks (and possible gluon radiation), and a momentum imbalance due to the undetected neutrino. Furthermore, the $b$ jets can sometimes be distinguished from light quark and gluon jets, as described in section 3.4.

The largest background comes from the direct production of $W$ bosons in association with significant jet activity. An example of this background, which we will refer to as $W+\mathrm{QCD}$, is shown in figure 1.2 .

We also note that dilepton and tau events can produce a lepton + jets signature. In $t \bar{t}$ Monte Carlo simulations (described later), about $4 \%$ of the events in the $W+\geq 3$ 


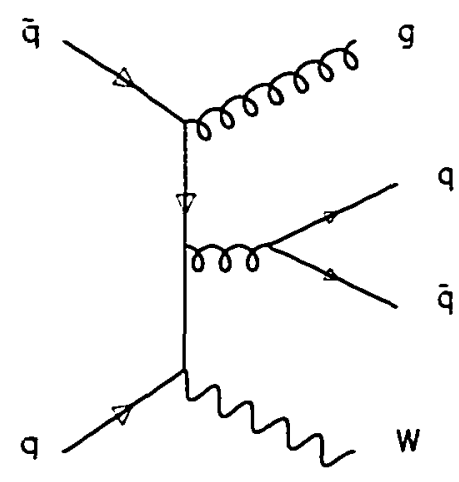

Figure 1.2: An example of $W+$ multijet production.

jets sample come from dilepton modes where the second lepton is not identified, and about $8 \%$ of the events come from tau modes, where either the hadronic tau decay is taken as a jet, or the primary lepton results from the decay of the tau.

\subsection{Motivation to Search for Hadronic Boson Decays}

Since hadronic boson decays result in pairs of jets with a known invariant mass, and the decay width is much smaller than the detector resolution, observation of these decays is useful to study both the calibration and resolution of the detector.

Uncertainty on the jet energy scale, the accuracy with which the measured calorimeter jet energies correspond with the original quark energies, is a large systematic uncertainty in many measurements at $\mathrm{CDF}$, especially the top mass measurement. Measurements of the $W$ mass in the hadronic mode provide a direct, in situ calibra- 
tion of the detector. This provides extra motivation to search for hadronic II decays in semileptonic $t \bar{t}$ events, because the calibration of the $W$ jets occurs inside the $t \bar{t}$ events themselves. Furthermore, the $b$ jets in top decays can be calibrated with the discovery of the decay mode $Z \rightarrow b \bar{b}$.

Improving jet energy resolution is also a major concern at CDF, especially in the search for the Higgs boson in the process $p \bar{p} \rightarrow V H, H \rightarrow b \bar{b}$, where $V$ is a $W$ or $Z$ boson which decays leptonically. Increasing the sharpness of the Higgs dijet mass peak is important in distinguishing the signal from the $V b \bar{b}$ background continuum [20]. Samples of hadronic $W$ and $Z$ decays provide a means to test new jet measurement techniques.

Identifying hadronic $W$ decays in semileptonic $t \bar{t}$ events is necessary to confirm the top quark discovery in this mode. It will also provide a clean $t \bar{t}$ sample, and will simplify kinematic reconstruction and study of the events. Methods used to search for the hadronic $W$ may also be used to search for nonstandard top decays of the form $t \rightarrow X b$, with $X \rightarrow j j$.

Finally, since these jets come from quarks, they may be used to study differences between quark jets and gluon jets [21]. Gluon jets are expected to have more particles, and shower wider than quark jets. 


\section{Chapter 2}

\section{Experimental Setup}

The Collider Detector at Fermilab (CDF) is a general purpose detector for study of $p \bar{p}$ collisions at Fermilab's Tevatron collider. The data for this analysis were taken at a center-of-mass energy of $1.8 \mathrm{TeV}$. Run $1 \mathrm{~A}$ consisted of $19.3 \mathrm{pb}^{-1}$ of data taken from 1992 and 1993, and Run $1 B$ and Run $1 B$ took $80 \mathrm{pb}^{-1}$ in 1994 and 1995 . A brief run at $\sqrt{s}=630 \mathrm{GeV}$ took place in late 1995 to early 1996 , followed by another run at $\sqrt{s}=1.8 \mathrm{TeV}$, Run $1 \mathrm{C}$, which took $3 \mathrm{pb}^{-1}$ with only partial tracking information.

The CDF detector has azimuthal symmetry as well as forward-backward symmetry. When describing the detector, we take the $z$ direction to be along the proton beam, the polar angle $\theta$ to be measured from the proton beam, and $\phi$ to be the azimuthal angle about the beam. We define the pseudo-rapidity $\eta$ to be $-\ln (\tan (\theta / 2))$. Unless otherwise noted, $\eta$ is calculated by taking $z=0$. We use the symbol $E$ to represent energy measured by the calorimetry, and the symbol $\vec{P}$ to represent momentum, measured by tracking chambers. We also define the transverse energy $E_{T}$ to be the projection of the observed energy $E$ in the direction perpendicular to the beam axis $\left(E_{T}=E \sin \theta\right)$, and, similarly, the transverse momentum $P_{T}=P \sin \theta$. 
The invariant mass.$M$ of a system is defined as $\sqrt{\left(\sum_{i} E_{i}\right)^{2}-\left|\sum_{i} \vec{P}_{i}\right|^{2}}$.

The detector surrounds the interaction point with layers of different detector components. The detector has been described in full detail elsewhere [22]. A cross section of the detector is shown in figure 2.1 .

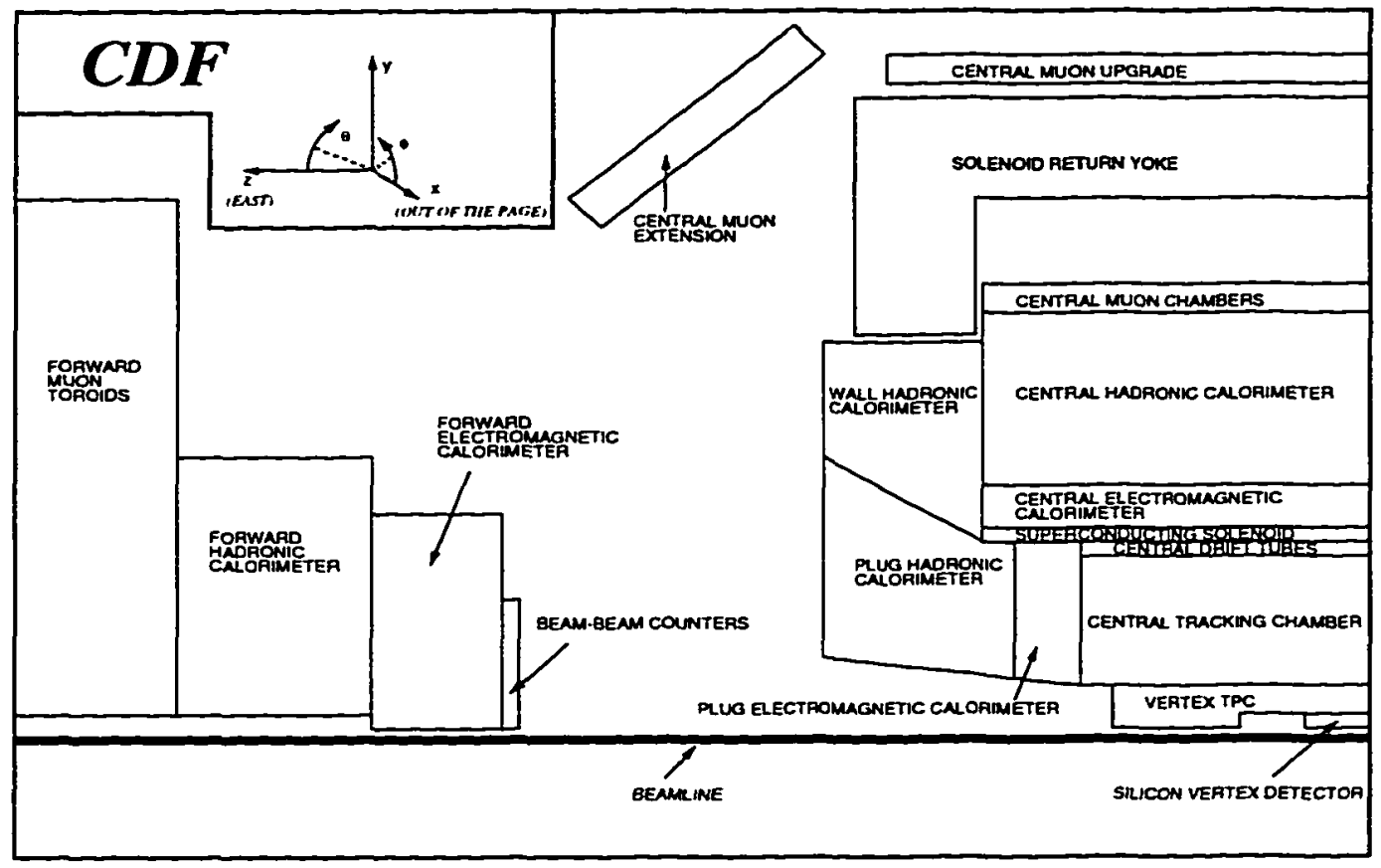

Figure 2.1: A cross section of the CDF detector.

\subsection{The Tevatron Accelerator}

Fermilab's Tevatron collider is currently the world's highest energy accelerator. It collides counter-rotating bunches of protons and antiprotons at a center-of-mass energy of $1.8 \mathrm{TeV}$. The Tevatron is in a circular tunnel $6300 \mathrm{~m}$ in circumference near Batavia, IL. Superconducting dipole magnets are used to keep the particles in a circular orbit, and radio-frequency $(\mathrm{RF})$ cavities provide the acceleration. The same 
magnets are used to contain the protons and antiprotons. These magnets have a maximum dipole bending field of $4.4 \mathrm{~T}$. A schematic of the accelerator complex is provided in figure 2.2 .

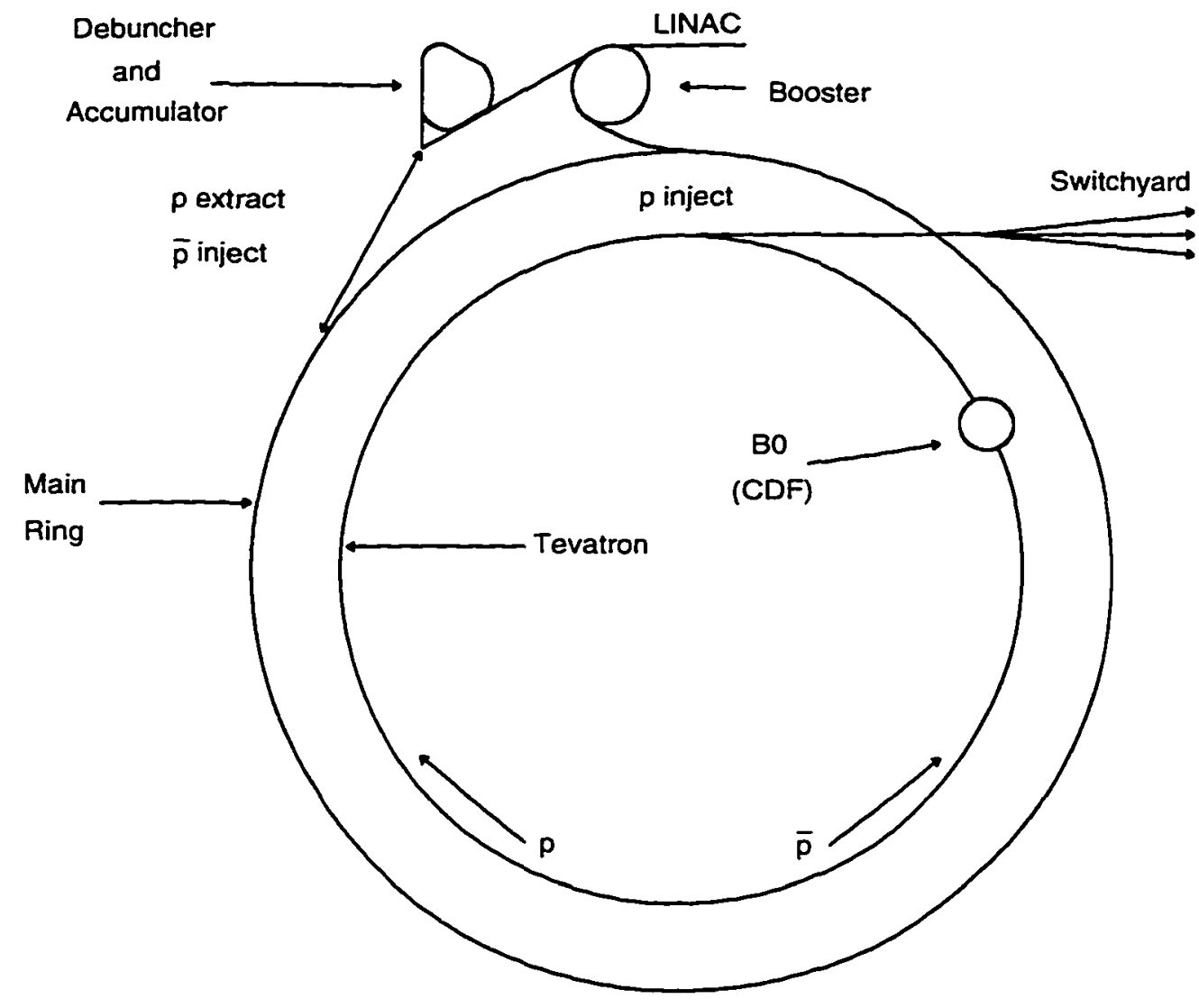

Figure 2.2: A schematic of the accelerator complex. The CDF detector is located at the $\mathrm{B} 0$ interaction point.

The protons used are obtained from a bottle of hydrogen gas. Extra electrons are added to the hydrogen atoms, forming $\mathrm{H}^{-}$ions. The $\mathrm{H}^{-}$ions are accelerated to an energy of $750 \mathrm{keV}$ by a Cockroft-Walton electrostatic accelerator, and then to an energy of $400 \mathrm{MeV}$ by a linear accelerator (LINAC). Then, the electrons are stripped off, leaving a bare proton. The protons are accelerated to $8 \mathrm{GeV}$ in a 
circular accelerator $475 \mathrm{~m}$ in circumference called the Booster. Proton bunches from the Booster are next injected into the Main Ring, which shares the same tunnel as the Tevatron, and accelerates the particles to $150 \mathrm{GeV}$, at which point they are switched into the Tevatron and accelerated to $900 \mathrm{GeV}$.

The antiprotons are created by colliding Main Ring protons with a fixed tungsten target. Antiprotons are collected from the resulting debris, and focused, cooled, and stored at energies of $8 \mathrm{GeV}$ in the Accumulator, until there are enough to inject into the Main Ring and Tevatron.

The protons and antiprotons in the Tevatron are grouped into six bunches. The bunches collide at the $\mathrm{B} 0$ and $\mathrm{D} 0$ interaction points every $3.5 \mu \mathrm{s}$, with a typical luminosity of $8 \times 10^{30} \mathrm{~cm}^{-2} \mathrm{~s}^{-1}$.

\subsection{Tracking Chambers}

The inner layers of the detector consist of tracking chambers. They operate in a uniform magnetic field of $1.4 \mathrm{~T}$, provided by a superconducting magnetic coil.

The protons and antiprotons collide in a vacuum maintained by a thin beryllium beampipe, with a radius of $1.9 \mathrm{~cm}$. The beampipe is surrounded by the silicon vertex detector (SVX), a microstrip detector designed to observe secondary decays of longlived particles. Outside the SVX is the Vertex Time Projection Chamber (VTX), which gives information on the $z$ position of the event vertex. The Central Tracking Chamber (CTC) is a large cylindrical drift chamber used to measure the momenta of charged tracks. 


\subsubsection{Central Tracking Chamber (CTC)}

The CTC is a $1.3 \mathrm{~m}$ radius $3.2 \mathrm{~m}$ long cylindrical drift chamber which gives precise momentum measurements in the angular region $40^{\circ}<\theta<140^{\circ}(-1<\eta<1)$. with a resolution better than $\delta P_{T} / P_{T}^{2} \leq 0.002(\mathrm{GeV} / \mathrm{c})^{-1}$. It consists of $150 \mathrm{~km}$ of wire arranged in 84 layers, which in turn form 9 superlayers (fig. 2.3). Five of the superlayers have 12 layers of sense wires running parallel to the beam. These superlayers are interleaved with four stereo superlayers, which consist of six sense wires in which the angle between the wire and the beam alternates between $\pm 3^{\circ}$. These stereo superlayers give a $z$ resolution of $4 \mathrm{~mm}$. The wires are kept in a gas mixture of argon, ethane, and ethanol $(49.6 \%, 49.6 \%$, and $0.8 \%$, respectively).

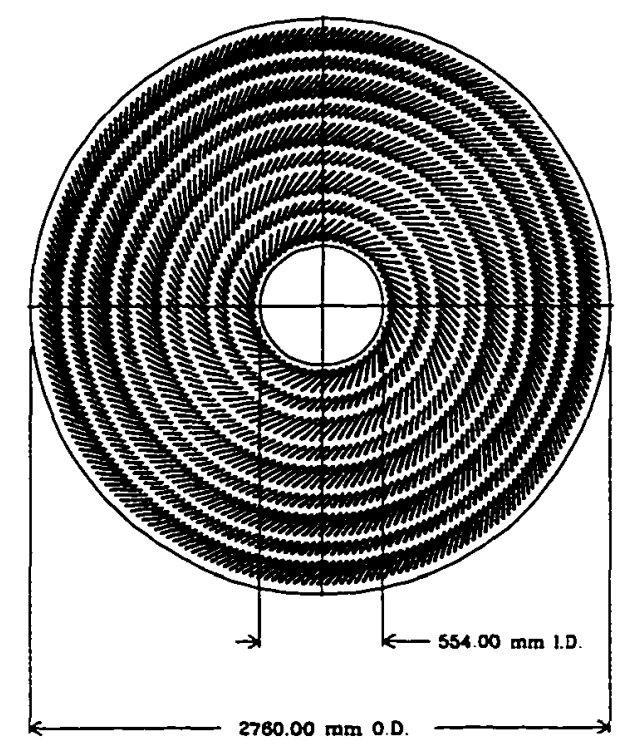

Figure 2.3: An end view of the Central Tracking Chamber.

The uses of the CTC in this analysis are first, to measure the momenta of lepton candidates, and, second, to provide seed tracks for VTX and SVX tracking. 


\subsubsection{Vertex Time Projection Chamber (VTX)}

The Vertex Time Projection Chamber (VTX) provides tracking in the $r-z$ plane. It provides a measurement of the $z$-position of the primary vertex, the $p \bar{p}$ interaction, with a resolution of $1 \mathrm{~mm}$. The VTX also provides $z$ information to CTC tracking, and is used to reject electrons from photon conversion.

The VTX is an octagonal drift chamber consisting of eight modules of radial sense wires in an argon-ethane gas mixture. It extends to a radius of $22 \mathrm{~cm}$.

\subsubsection{Silicon Vertex Detector (SVX)}

The CDF Silicon Vertex Detector (SVX) provides high-resolution $r-\phi$ tracking information just outside the $1.9 \mathrm{~cm}$ radius of the beryllium beam pipe. The SVX allows precise measurement of the $x$ and $y$ coordinates of the primary vertex, and, more importantly, allows detection of tracks which do not point back to the primary vertex, indicating long-lived decays. The SVX single hit resolution is measured to be $13 \mu \mathrm{m}$, and the impact parameter resolution for high-momentum tracks is measured to be $17 \mu \mathrm{m}$.

The SVX consists of four concentric cylindrical layers of silicon microstrip detectors, at radii of $3.0,4.2,6.8$, and $7.9 \mathrm{~cm}$. Two barrels, consisting of twelve wedges of silicon strips, extend out to $|z|=25 \mathrm{~cm}$, with a $2.15 \mathrm{~cm}$ gap between them. Since the $z$-coordinate of the interaction point has a standard deviation of $30 \mathrm{~cm}$, the SVX covers about $60 \%$ of the interactions. A drawing of an SVX barrel is shown in figure 2.4 


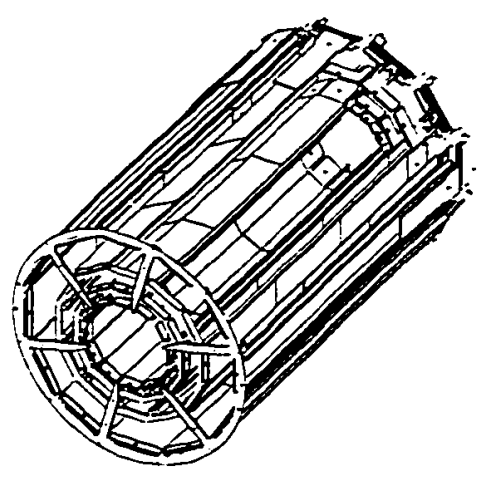

Figure 2.4: One barrel of the SVX.

Each silicon strip detector is a $300 \mu \mathrm{m}$ thick crystal of silicon, with aluminum readout strips on top, parallel to the beam. The readout strips are spaced every 60 $\mu \mathrm{m}$ in the three inner layers, and every $55 \mu \mathrm{m}$ in the outer layer. Incident charged particles leave ionization charge in the strips. Adjacent strips with significant charge are grouped into clusters.

SVX tracking is achieved by assigning SVX hits to CTC tracks. We accept only those SVX tracks which increase the $\chi^{2}$ of the combined SVX-CTC track by no more than six. This cut is $96 \%$ efficient. We also require that at least two of the SVX hits to be "good" hits, that is, not shared with another CTC track, containing no SVX channels with low gain or high noise, and having a charge profile consistent with only one particle. The two-good-hit requirement is satisfied for $78 \%$ of SVX tracks in generic jet triggers, independent of track $P_{T}$. The SVX-CTC track has a momentum resolution of $\delta P_{T} / P_{T}=0.0009 P_{T} \oplus 0.0066$ 
The SVX detector used in Run 1.A [23] suffered significant radiation damage during the run, and was replaced with a similar detector with radiation-hard electronics for Run 1B [24].

\subsection{Calorimetry}

The calorimetry at CDF is arranged in a projective "tower" geometry covering up to $\eta$ of 4.2. Each tower consists of an electromagnetic shower counter in front of a hadron calorimeter. The four calorimetry sections, the central, endwall, plug, and forward, are shown in figs 2.1 and 2.5. The towers each cover 0.1 units of $\eta$ and $15^{\circ}$ (central region) or $5^{\circ}$ (plug and forward region) in $\phi$. Table 2.1 lists the energy resolutions.

\begin{tabular}{||c|c|c|c||}
\hline Calorimeter & $|\eta|$ Range & Energy Resolution & Thickness \\
\hline CEM & $0-1.1$ & $13.7 \% / \sqrt{E_{T}} \oplus 2 \%$ & $0.3 \Lambda, 18 X_{0}$ \\
CHA & $0-0.9$ & $50 \% / \sqrt{E_{T}} \oplus 3 \%$ & $4.5 \Lambda$ \\
WHA & $0.6-1.1$ & $75 \% / \sqrt{E} \oplus 4 \%$ & $4.5 \Lambda$ \\
\hline PEM & $1.1-2.4$ & $22 \% / \sqrt{E} \oplus 2 \%$ & $1.0 \Lambda, 19 X_{0}$ \\
PHA & $1.3-2.4$ & $106 \% / \sqrt{E} \oplus 6 \%$ & $5.7 \Lambda$ \\
\hline FEM & $2.2-4.2$ & $26 \% / \sqrt{E} \oplus 2 \%$ & $0.8 \Lambda, 25 X_{0}$ \\
FHA & $2.4-4.2$ & $137 \% / \sqrt{E} \oplus 3 \%$ & 7.7 .1 \\
\hline
\end{tabular}

Table 2.1: Energy resolutions of the various calorimeter components. 


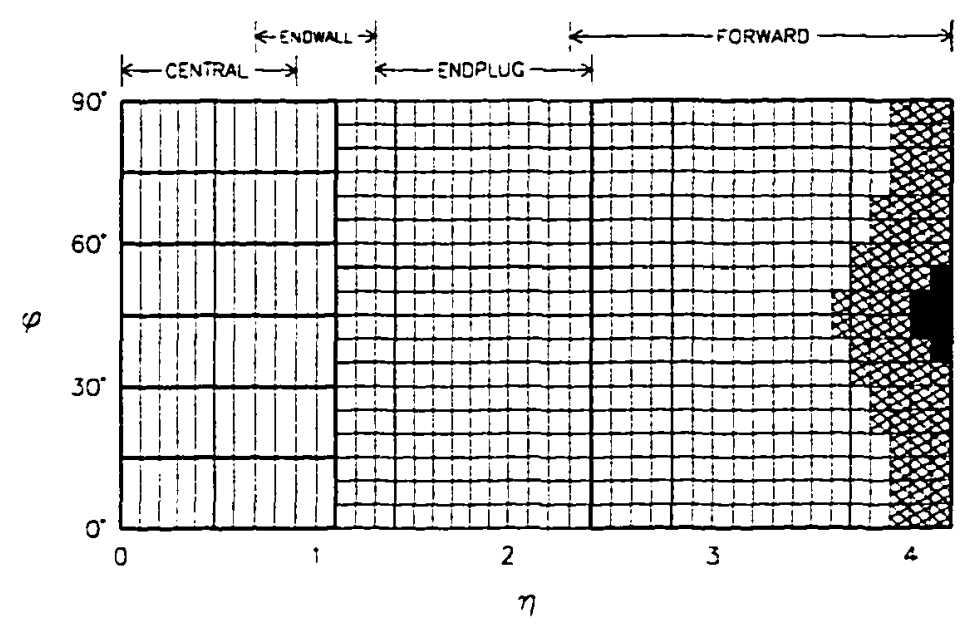

Figure 2.5: The tower segmentation of the CDF calorimetry.

The central and plug section are used in this analysis, and are described individually below.

\subsubsection{Central Calorimetry (CEM, CHA, WHA)}

The Central Electromagnetic Calorimeter (CEMI) consists of 18 radiation lengths of alternating layers of 0.14 in thick absorbers, made of lead cased in aluminum, and 5 mm thick polystyrene scintillator.

Embedded in the CEM at a depth of five radiation lengths is the Central Electro- 
magnetic Strip Chamber (CES), a set of wire proportional chambers which measures the shower profile at shower maximum in both the $z$ and $r-\dot{\phi}$ view, and is used in electron and photon identification.

Just in front of the CEM is the Central Preradiator (CPR), a set of multiwire proportional chambers used to sample electromagnetic showers that begin in in the solenoid magnet material $\left(1.075 X_{0}\right)$.

Behind the CEM are the Central Hadron Calorimeter (CHA) and the Endwall Hadron Calorimeter (WHA). Both use layers of iron absorbers $(2 . \overline{\mathrm{cm}}$ thick in the $\mathrm{CHA}$, and $5 \mathrm{~cm}$ thick in the WHA) and acrylic scintillators ( $1 \mathrm{~cm}$ thick).

\subsubsection{Plug Calorimetry (PEM, PHA)}

Because of the high radiation in the more forward detector regions, the plug and forward calorimeters are gas calorimeters, consisting of alternating lavers of absorbers and gas proportional tubes. The gas used is a $50-50$ mixture of argon and ethane, with a small amount of alcohol added to prevent glow discharge. The Plug Electromagnetic Calorimeter uses 0.27 thick lead sheets as absorbers, and $0.7 \mathrm{~cm}$ thick proportional tubes. The Plug Hadron Calorimeter (PHA) uses $5.1 \mathrm{~cm}$ thick steel absorbers, and $1.4 \mathrm{~cm}$ thick proportional tubes.

\subsection{Muon Detection}

CDF has three sets of muon chambers, the Central . Iuon Chambers (CAIU), the Central Muon Upgrade (CMP), and the Central Muon Extension (C.MX). Each con- 
sists of four layers of drift chambers placed outside the calorimetry. Hits in the muon chambers are fitted to line segments, called stubs, which are then matched to CTC tracks. The momentum measurements for the muons are taken from the CTC tracks. The muon detectors are described below.

\subsubsection{Central Muon Chamber (CMU)}

The CMU consists of forty-eight wedges placed behind the CHA. The CHA and the magnet yoke provide six interaction lengths of shielding. The CMU covers $84 \%$ of the solid angle within $|\eta|<0.6$.

\subsubsection{Central Muon Upgrade (CMP)}

The Central Muon Upgrade consists of another $0.6 \mathrm{~m}$ of steel shielding ( $3 . \overline{5}$ radiation lengths), followed by another layer of drift chambers. The CMP covers roughly the same $\eta$ range as the CMU and covers $63 \%$ of the solid angle. $54 \%$ of the solid angle is covered by both the CMU and CMP.

\subsubsection{Central Muon Extension (CMX)}

Muon coverage has been extended out to $|\eta|$ of 1.0 by the addition of the Central Muon Extension, four free-standing arches supporting additional drift chambers, as well as scintillator counters for triggering. The CMX covers $71 \%$ of the solid angle within $0.6<|\eta|<1.0$, and provides an additional $10 \%$ muon acceptance for these analyses. 


\subsection{Triggers}

CDF's trigger is a three-level system designed to reduce the rate of events from the beam-crossing frequency of $286 \mathrm{kHz}$ to a rate which can be written to tape.

\subsubsection{Beam-Beam Counters (BBC's)}

The BBC's are planes of scintillator near the beams in the far forward and backward regions, $5.8 \mathrm{~m}$ from the center of the detector. They are used to signal whether a $p \bar{p}$ interaction has occured during a beam crossing. This provides a basic trigger requirement, and is also used to measure beam luminosity.

\subsubsection{Level 1}

Since digitization of signals takes $\approx 1 \mathrm{~ms}$, Level 1 and 2 trigger decisions are made on analog signals. The Level 1 trigger searches for high $E_{T}$ single calorimeter towers, and looks for track segments in the central muon chambers. The Level 1 system incurs no dead time.

\subsubsection{Level 2}

The Level 2 system adds a hardware track processor, calorimeter clustering, and global calorimeter summing. The detector signals are held for the $\approx 20 \mu s$ needed to make a Level 2 decision, so some dead time is incurred. Events pass Level 2 at a rate of about $12 \mathrm{~Hz}$.

The track-finding hardware, the Central Fast Tracker (CFT), identifies CTC tracks 
from analog outputs. The CFT does tracking with a resolution $\frac{\delta P_{T}}{P_{T}} \approx 0.035 \times P_{T}$. and has an efficiency of $93.5 \pm 0.3 \%$ for tracks with $P_{T}>10 \mathrm{GeV} / \mathrm{c}$. The tracking information is then used for electron and muon triggers.

Level 2 calorimeter clustering uses two thresholds for combined electromagnetic and hadronic tower $E_{T}$, a "seed" threshold of $3 \mathrm{GeV}$, and a lower "shoulder" threshold of $1 \mathrm{GeV} . A$ cluster is defined as a tower over the seed threshold, plus any contiguous chain of towers over the shoulder threshold.

Global calorimeter summing is used for triggers based on summed $E_{T}$ and missing $E_{T}$.

\subsubsection{Level 3}

The highest trigger level, Level 3, works from digital signals and is processed on a cluster of SGI workstations, using algorithms identical to those used in the subsequent "offline" analysis. Events passing these triggers are written to tape to await offline reconstruction.

\subsection{Offline Reconstruction}

Offline event reconstruction converts the raw detector signals on tape into physics objects. The events are then sorted and written to tape in both full (DST) and reduced (PAD) form. Processing is done by feeding events in parallel to a "farm" of 64 SGI and 37 IBM workstations. The capacity of the system is about 1.3 million events per week. Eighty million data events were written out by the CDF experiment 
between 1992 and 1995. 


\section{Chapter 3}

\section{Event Reconstruction}

\subsection{Jets}

\subsubsection{Clustering}

CDF jet clustering is done using a cone of fixed radius $R$ in $\phi-\eta$ space about the cluster centroid, because jets are approximately circular in $\phi-\eta$ space. For many analyses, a radius of $R=\sqrt{(\Delta \phi)^{2}+(\Delta \eta)^{2}}=0.7$ is chosen. Top quark analyses. because of their high jet activity and higher- $E_{T}$ jets, use $R=0.4$.

The clustering algorithm first looks for seed towers with $E_{T}>1.0 \mathrm{GeV}$. In the plug and forward region, towers are grouped in sets of three in $\phi$, to match central segmentation. Starting with the first seed tower in $\phi$, preclusters are then formed from unbroken chains of neighboring seed towers with continuously decreasing $E_{T}$ : within a $7 \times 7$ window of towers of the original seed tower.

Next, a cluster is formed by including all towers with $E_{T}>100 \mathrm{MeV}$ within $R$ of the precluster centroid. A new cluster centroid is calculated, and the process is iterated until the tower list remains unchanged. 
If there is overlap between clusters, the clusters are merged if more than $75 \%$ of the $E_{T}$ of a cluster is shared. If overlapping clusters are not merged, then each tower is assigned to the nearest cluster. Again, this process is continued iteratively until a stable result is achieved.

When a cluster is found, the energy $E$ is defined as the scalar sum of all tower energies in the cluster, and the momentum $\vec{P}$ by the vector sum. These vectors, unless otherwise noted, will be calculated with respect to the the $z$ position of the primary event vertex, as measured by the VTX.

\subsubsection{Corrections}

The energy deposited in the calorimeter may vary from the true parton energy for many reasons. Energy may be lost by detector non-linearities and cracks. Jet fragmentation and the magnetic field cause particles to spread outside the clustering cone. Neutrinos and muons will deposit little or no energy. Energy not associated with the hard scattering process will be collected in the cones, as will energy from multiple $p \bar{p}$ interactions. We correct for these effects [25], so that our measured jet energies correspond as closely as possible to the original parton energies. Uncorrected energies will also be referred to as raw energies.

The first step in correcting these effects is to determine the absolute correction for the central calorimetry. This is done using a Monte Carlo technique, with jet fragmentation and calorimeter pion response tuned to CDF data. Next, the corrections for central jets are extended to other regions of $\eta$ by studying $E_{T}$ balance in dijet events 
where one jet is central. Finally, extra corrections are added for out-of-cone losses. underlying event, and multiple interactions. The out-of-cone correction is dependent on the $E_{T}$ of the jet, and is derived from Monte Carlo, and the underlying event and multiple interaction corrections are derived from data. The summed corrections typically add about $30 \%$ to jet energies.

For top quark analyses, extra corrections are required [26]. Bottom and charm quarks frequently decay semileptonically, producing electrons, muons and neutrinos. In addition, the high jet multiplicities frequently create situations where the losses of one jet fall into the cone of another. This is especially true for highly boosted hadronic $W$ decay, where the jets are produced close together.

These top-specific corrections are obtained by generating HERWIG Monte Carlo at $m_{t}=175 \mathrm{GeV} / c^{2}$, and matching the generated partons to the reconstructed jets. The corrections are done separately for jets from $W$ decay, jets from $b$ decay, and $b$ jets with electron and muon candidates from semileptonic decay modes, as identified in section 3.4.2. The correction factors are shown in figure 3.1.

All jet energy corrections are designed to produce the correct average $E_{T}$, not to reduce variations around the mean. 


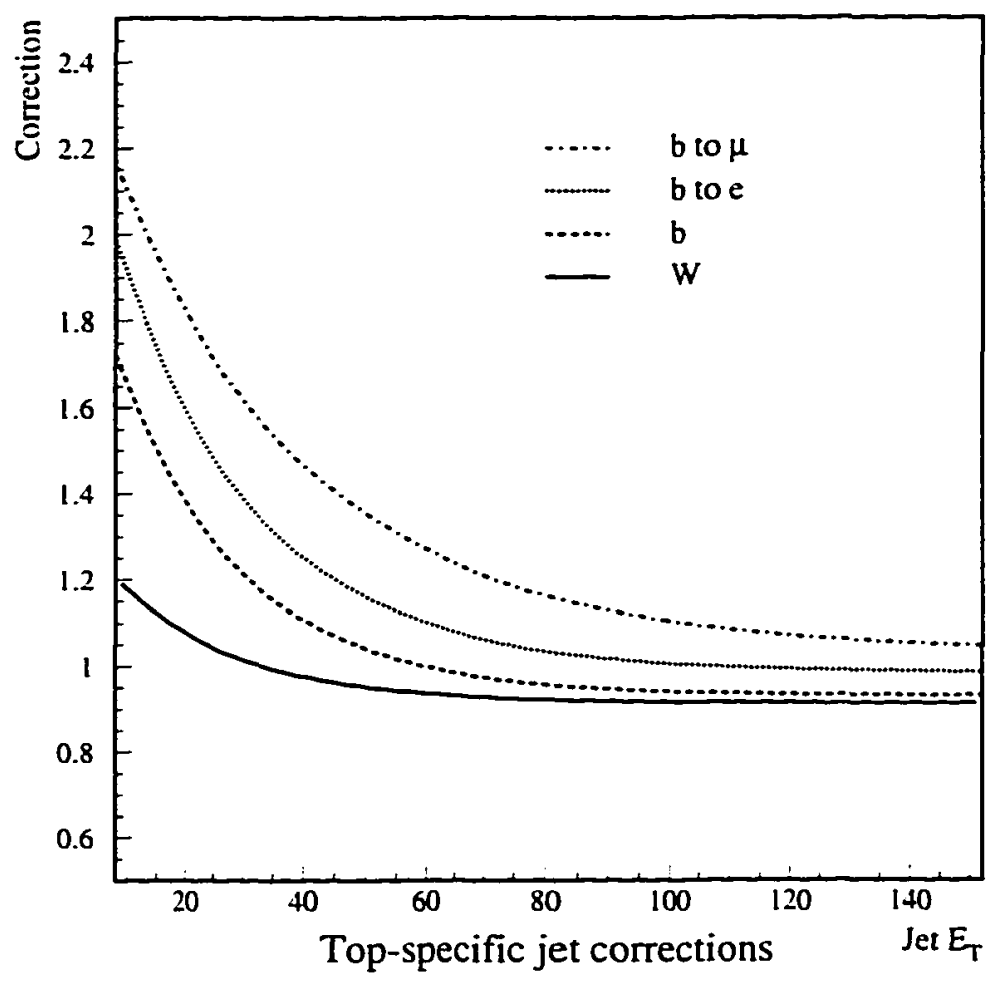

Figure 3.1: Top-specific correction factors to the Jet $E_{T}$

\subsection{High $P_{T}$ Lepton Selection}

\subsubsection{Electrons}

\section{Electron triggers}

The Level 2 central electron trigger requires a CEM cluster matched to a CFT track. where the ratio of hadronic to electromagnetic cluster energy HAD/EM is less than 0.125 . The requirements are shown in table 3.1. For Run 1B, the efficiency is combined with the $\not_{t}$ trigger described in section 3.3.1. 


\begin{tabular}{||c|c|c||}
\hline Cut & Run 1A & Run 1B \\
\hline L1 single tower $E_{T}$ & $6 \mathrm{GeV}$ & $8 \mathrm{GeV}$ \\
L2 cluster $E_{T}$ & $9 \mathrm{GeV}$ & $16 \mathrm{GeV}$ \\
CFT track $P_{T}$ & $9.2 \mathrm{GeV} / c$ & $12 \mathrm{GeV} / c$ \\
Seed tower threshold & $9 \mathrm{GeV}$ & $8 \mathrm{GeV}$ \\
Shoulder tower threshold & $7 \mathrm{GeV}$ & $7 \mathrm{GeV}$ \\
Efficiency & $(92.8 \pm 0.2) \%$ & $\approx 100 \%$ with $E_{t}$ trigger \\
\hline
\end{tabular}

Table 3.1: Central electron trigger requirements

\section{Electron offline cuts}

An electron candidate consists of an electromagnetic calorimeter cluster of three towers with a CTC track pointing at it. Central high $P_{T}$ electron candidates are required to have a calorimeter $E_{T}>20 \mathrm{GeV}$, and be in the region $\eta<1.0$. They must be mostly electromagnetic clusters, with the hadronic energy comprising less than $5 \%$ of the electromagnetic energy. The shower centroid must also be away from calorimeter module boundaries. The fiducial region covers $84 \%$ of the solid angle within $|\eta|<1.0$ To avoid tracking mismatches, the ratio of the calorimeter energy to the track momentum $E / P$ must be less than 1.8 .

Some backgrounds to electron identification include fluctuations of hadronic showers and photons from $\pi^{0}$ decays overlapping with hadron tracks. The following cuts help combat these backgrounds. 
The shower in the Central Electromagnetic Strip Chamber (CES) is used. The track position must match to the centroid of the CES shower, and the lateral shower profile in both the calorimeter and CES must be consistent (via a $\chi^{2}$ test) with test-beam electrons.

The $z$ vertex of the event must be within $60 \mathrm{~cm}$ of the center of the detector, and the $z$ position of the electron candidate track must be within $\overline{5} \mathrm{~cm}$ of the primary vertex. Also, a VTX track is required to match to the candidate track, to help eliminate photon backgrounds.

Another way to eliminate backgrounds from photon conversions, where $\gamma \rightarrow e^{+} e^{-}$, searches for an oppositely charged track very near to the candidate track.

We define the calorimeter isolation $I$ as the ratio of the summed $E_{T}$ in a cone of $R=0.4$ around the electron but excluding the electron cluster to the electron cluster $E_{T}$. We require $I<0.1$.

These cuts are measured to be $(74 \pm 1) \%$ efficient $[27]$.

\subsubsection{Muons}

\section{Muon triggers}

The Level 1 central muon triggers look for a CMU track stub with $P_{T}>6 \mathrm{GeV} / c$. or a C.MX stub with $P_{T}>10 \mathrm{GeV} / c$. Level 2 requires a track $P_{T}$ of $9.2 \mathrm{GeV} / c$ for Run $1 \mathrm{~A}$ and $12 \mathrm{GeV} / \mathrm{c}$ for Run 1B. In Run $1 \mathrm{~B}$, muon triggers were prescaled, set to randomly reject events to reduce trigger rate, unless there was a separate jet cluster of $E_{T}>15 \mathrm{GeV}$. Level 3 makes a track-matching requirement and requires 
track $P_{T}>18 \mathrm{GeV} / c$. The trigger efficiencies are measured to be $(8.3 .8 \pm 1.9) \%$ for Run $1 \mathrm{~A}$ and $(87.2 \pm 1.4) \%$ for Run $1 \mathrm{~B}[28]$, where the Run $1 \mathrm{~B}$ number includes the $\not{ }_{t}$ trigger described below.

\section{Muon offline cuts}

Muon candidates are CTC tracks which point at two or more hits in the muon chambers. For $W$ and top analyses, CMIX muons are excluded as primary leptons. The muon must have track $P_{T}>20 \mathrm{GeV} / \mathrm{c}$, and the track must point to the muon chamber hits. The electromagnetic and hadronic calorimeter energies in the tower at which the track points must have at least $0.1 \mathrm{GeV}$ of summed $E_{T}$, but they may not exceed 2 and $6 \mathrm{GeV}$, respectively. The track is required to match to the C.MU muon stub within $2 \mathrm{~cm}$, and within $5 \mathrm{~cm}$ for CMP stubs. As with electrons, the tracks are required to match in $z$ within $5 \mathrm{~cm}$ of the primary vertex, and have calorimeter isolation $I<0.1$.

To reduce background from cosmic ray muons, we demand that the muon track's impact parameter $d$, the track's distance of closest approach to the primary event vertex in the $x y$ plane, be less than $3 \mathrm{~mm}$.

The efficiency of the offline cuts is $(92.4 \pm 1.4) \%[27]$.

Muon backgrounds can arise from hadrons which punch through the calorimeter and shielding, and from decay-in-flight of pions and kaons. 


\subsection{Missing $E_{T}$}

Neutrinos from $W$ decays do not interact with the detector, and their presence is only inferred from momentum imbalance in the transverse direction. Momentum balance parallel to the beams cannot be measured, since the interacting partons carry an unknown fraction of the proton or antiproton energy, and many fragmentation products continue along the beampipe direction and are not detected.

The missing $E_{T}\left(E_{t}\right)$ is defined as the negative vector sum of all calorimeter tower $E_{T}$ 's over a small, detector-dependent threshold with $|\eta|<3.6$. Corrections are made to the $Z_{t}$ for high- $P_{T}$ muons. CDF $W$ analyses require $\not_{t}>20 \mathrm{GeV}$.

For top mass analyses, corrections are made to the $\not_{t}$. These will be described in section 4.10 .2 .

\subsubsection{Missing $E_{T}$ trigger}

For Run 1B, Level 2 triggers based on $Z_{t}$ were used for $W$ event selection. One trigger, designed for $W \rightarrow e \nu$ events, looks for $\not_{t}>20 \mathrm{GeV}$, along with a CEM cluster with $E_{T}>16 \mathrm{GeV}$ and a CES profile consistent with electrons or photons. It trigger for $W \rightarrow \mu \nu$ events demands $Z_{t}>35 \mathrm{GeV}$ along with a central calorimeter cluster. 


\subsection{Heavy Flavor Tagging}

\subsubsection{Secondary Vertex Tagging}

The $b$ hadrons produced in $t \bar{t}$ events have typical decay lengths of a few millimeters. The most effective way to identify heavy quarks at CDF is the secondary vertex finding algorithm SECVTX, which uses the SVX detector to find long-lived particles by searching for track vertices significantly displaced from the primary event vertex of the $p \bar{p}$ interaction. It consists of two passes. The first pass uses looser track quality cuts to find vertices of three or more displaced tracks, and the second pass uses tighter track cuts to find two-track vertices.

We define track displacement in terms of the impact parameter $d$, and its measurement uncertainty $\sigma_{d}$. A track is said to have a positive impact parameter if $d$ points in the same hemisphere as the jet axis, and a negative impact parameter otherwise. Since long-lived particles will travel along the jet direction before decaying, we expect most of their displaced tracks to have a positive signed impact parameter. Negative $d$ tracks are expected to come more from measurement uncertainties on tracks originating at the primary vertex. We also define the two-dimensional decay length $L_{x y}$ to be the displacement of a vertex in the $x y$ plane. Figure 3.2 shows a displaced vertex, and labels $d$ and $L_{x y}$.

All tracks considered by this algorithm must be displaced, that is, they must have an impact parameter $d$ with respect to the primary vertex with a significance $\left|d / \sigma_{d}\right|>2.5$. They also must pass CTC-SVX track quality cuts, and survive an 


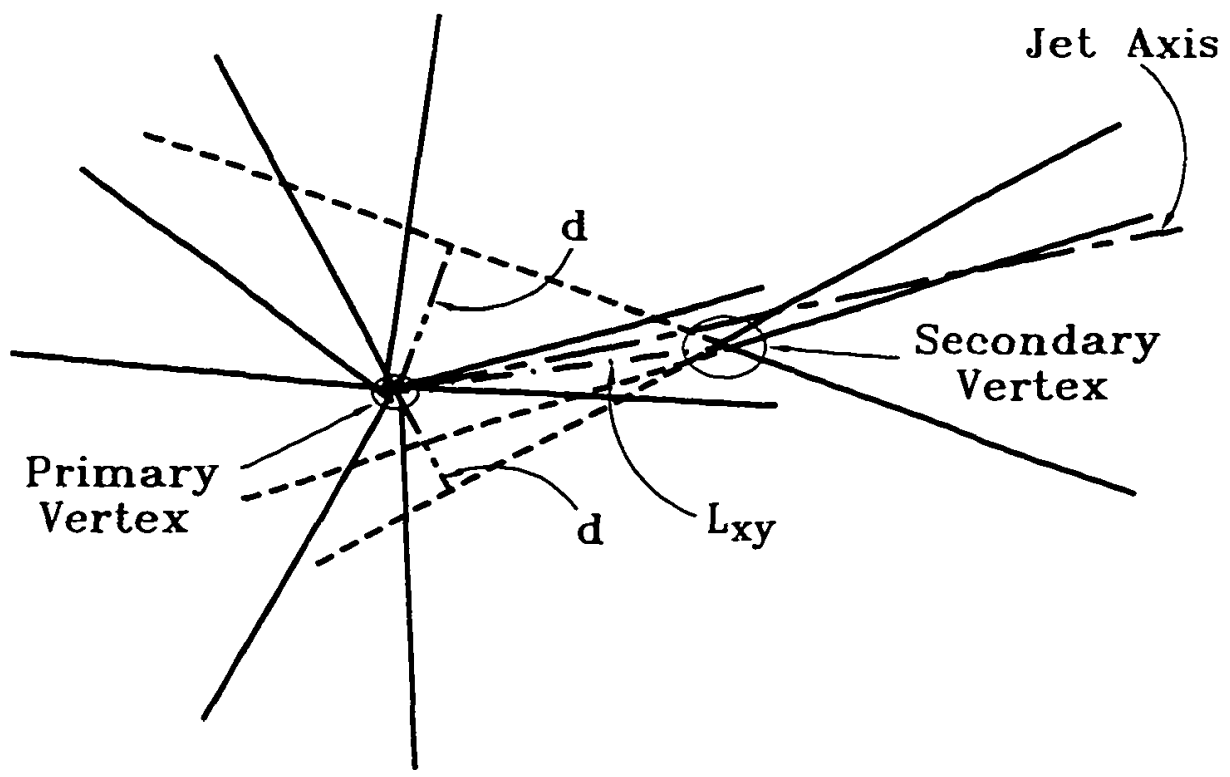

Figure 3.2: Simplified view in the $x y$ plane of SVX tracks forming a displaced vertex.

algorithm which looks for track pairs consistent with $K_{s}$ or $\Lambda$ particles.

The loose set of SVX track cuts for pass 1 require that tracks with three or four hits must have $P_{T}>0.5 \mathrm{GeV} / \mathrm{c}$ and at least one good cluster. A good cluster is defined to be a cluster associated with only one track, and made of three or fewer strips. Two-hit tracks are also accepted if both clusters are good, $P_{T}>1.5 \mathrm{GeV} / \mathrm{c}$, and the hits are either both in the inner two layers, or both in the outer two layers.

The tighter cuts of pass 2 require an impact parameter significance $\left|d / \sigma_{d}\right|>3.0$. and $P_{T}>1.5 \mathrm{GeV} / \mathrm{c}$. Four-hit tracks must have one good cluster, and three-hit tracks must have two good clusters. Two-hit tracks are rejected.

The SECVTX algorithm then attempts to form vertices from the tracks in each jet. It begins pass 1 by forming a seed vertex from the two best tracks. It then 
searches for a third track to add to this vertex. If a third track cannot be found, then the algorithm moves on to the next track pair, and forms a seed vertex from these tracks. If a third track is found, then SECVTX tries to add as many more tracks as possible. This procedure stops once the first vertex with three or more tracks is found.

If no vertex is found in pass 1 , two-track vertices are searched for in pass 2 .

If a vertex is found, it is required to lie inside the inner layer of silicon, to include a track with $P_{T}>2.0 \mathrm{GeV} / c$, and to have a decay length significance $L_{x y} / \sigma_{L I y}$ greater than 3.0. The typical accuracy on the decay length is $\approx 130 \mu \mathrm{m}$, much smaller than the distance traveled by the $b$ hadron. Jets with vertices satisfying these requirements are said to SECVTX-tagged.

A more complete study of this algorithm can be found in Ref. [29].

\subsubsection{Soft Lepton Tagging}

We also attempt to identify heavy quarks through the leptonic decays $b \rightarrow \ell \nu_{\ell} c$, where $\ell=e$ or $\mu$, or $b \rightarrow c \rightarrow \ell \nu_{\ell} X$. A jet is said to have a soft lepton tag, or an SLT-tag, if there is a track with $P_{T}>2 \mathrm{GeV} / \mathrm{c}$ consistent with being an electron or muon within its cone radius.

To identify electron tracks within jets, we attempt to match each CTC track with a CES shower. The CES shower is then required to be consistent, via a chi-squared test. with the size and shape expected from test beam electrons. Further electron track quality cuts include the specific ionization $\frac{d E}{d X}$, which suppresses hadron background. 
and CPR energy deposition, which discriminates against photon conversion. Also. the calorimeter tower at which the track points is required to have an energy consistent with the track momentum $(0.7<E / P<1.5)$, and to be mostly electromagnetic $(\mathrm{HAD} / \mathrm{EM}<0.1)$. If the track points to within $2 \mathrm{~cm}$ of a tower boundary, the neighboring tower is also included in calculating these quantities.

To identify muon tracks, we attempt to match CTC tracks with track segments in the CMU, CMP, or CMX. For very low $P_{T}$ central tracks $\left(2<P_{T}<3,|\eta|<0.6\right)$. we expect muons to be stopped by the steel shielding between the CMU and C.MP. so we only require $\mathrm{CMU}$ track segments. For higher $P_{T}$ tracks, we require matching segments in both the CMU and CMP.

A more complete study of the SLT algorithm can be found in Ref. [30].

\subsubsection{Jet Probability Tagging}

Another method which uses SVX tracks to search for long-lived particles is the jet probability algorithm [31]. This technique converts the impact parameter significance of good SVX tracks into a probability that the track is consistent with the primary vertex. Then, the "track probabilities" for all SVX tracks in a jet are combined to form the "jet probability", the probability that the jet is consistent with the primary vertex.

The strength of this technique lies in the fact that the jet probability provides a simple parameter which can be used to loosen or tighten tagging requirements. Also, since it doesn't require vertexing, it can tag jets with just one displaced track. 
The SVX track selection is similar to the "pass 2 " SECVTX cuts. except that no impact parameter significance is required. Tracks must have $P_{T}>1.5 \mathrm{GeV} / \mathrm{c}$. and have two good SVX clusters. Two-hit tracks are rejected if one of the hits is in the inner SVX layer.

The jet probability technique uses the impact parameter distribution from negative $d$ tracks to define the SVX resolution, and positive $d$ tracks to search for heavy flavor. The SVX resolution is defined for different classes of tracks, depending on the number of good hits and the total number of hits in the track. Then, the track probability for a positive $d$ track is defined to be the fraction of negative $d$ tracks of that class with an equal or greater i.p. significance. The track probabilities for all good tracks in the jet are combined to form the jet probability.

For tracks originating at the primary vertex, we expect the distribution of track probabilities to be flat from zero to one. Significantly displaced tracks will congregate at very low track probabilities. Similarly, jets whose origin is the primary vertex should have a flat distribution of jet probabilities, while an excess near zero indicates long-lived particles in the jet. The jet probability distributions for inclusive jets and a b-enriched sample of jets near muons is given in figure 3.3 .

For the subsequent analysis, we define a JP tag to correspond to a jet probability of less than $5 \%$. Note that this defines the mistag rate per taggable jet to be about $5 \%$. In the $W+\geq 3$ jet sample, $38 \%$ of jets not tagged by SECVTX are taggable. 

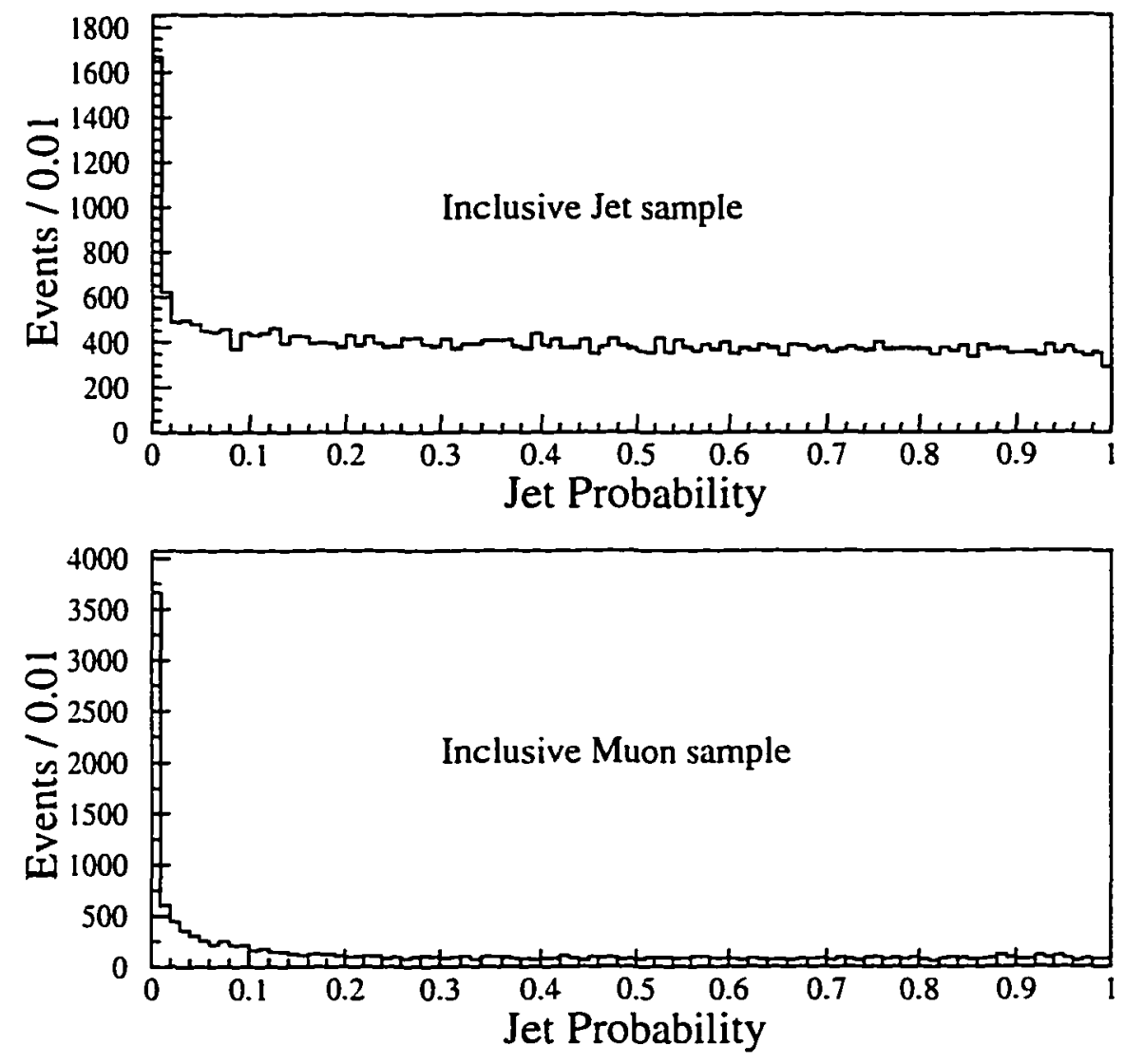

Figure 3.3: The distribution of jet probability for jets in the inclusive jet sample. and for jets near muon candidates. Low jet probability means the tracks are not consistent with the primary vertex. 


\begin{tabular}{|c|c|c|c||}
\hline Vethod & $b$ eff. (\%) & $c$ eff. (\%) & mistag rate (\%) \\
\hline SECVTX & $(27 \pm 3)$ & $(5.7 \pm 0.7)$ & $(0.3 \pm 0.1)$ \\
SLT & $(13 \pm 3)$ & $(6.8 \pm 0.5)$ & $(1.5 \pm 0.2)$ \\
SECVTX+SLT+JP & $(47 \pm 5)$ & $(18.4 \pm 1.7)$ & $(3.4 \pm 0.7)$ \\
\hline
\end{tabular}

Table 3.2: Tag rates for $b$ and $c$ jets, and mistag rates for the three taggers.

\subsubsection{Comparison of tagging efficiencies}

We now show the efficiencies of the taggers in $t \bar{t}$ events using Monte Carlo simulations, described later in section 4.2. Detailed comparison of Monte Carlo and data samples show that simulated tagging rates must be adjusted by a scale factor, largely due to the lower tracking efficiency in high-luminosity conditions. The scale factor for SECVTX and JP is $(0.826 \pm 0.07)$, and the scale factor for SLT is $(0.95 \pm 0.02)$. All Monte Carlo results stated hereafter include these scale factors.

We evaluate the tag rates for three types of jets: $b$ jets in $t \bar{t}$ events, $c$ jets from $W^{*}$ decay in $t \bar{t}$ events, and mistags in $W+Q C D$ background. The mistag rates have been studied in generic jet samples, and the largest uncertainty on them lies in estimating

the rate of heavy flavor in these samples. The tagging rates are shown in table 3.2 . We only apply the JP tagger to jets which were not tagged by SVX or SLT.

SECVTX and SLT tags are seen to be only weakly correlated [10]. 


\section{Chapter 4}

\section{Hadronic $W$ decays in $t \bar{t}$ events}

In this chapter, we show evidence of the hadronic decay of the $W$ boson in semileptonic $t \bar{t}$ decay. We use this signal to measure the mass of the $W$ boson and the top quark. We also show how this signal can be used to study the systematic uncertainties of the top mass measurement due to jet energy scale uncertainty and hard gluon radiation.

\subsection{Event Sample}

The search starts with the sample of $163 W+\geq 4$ jet events from the $109 \mathrm{pb}^{-1}$ combined dataset from Runs $1 \mathrm{~A}$ and $1 \mathrm{~B}$. The events come from electron, muon, or $Z_{t}$ triggers. The electron, muon, and $Z_{t}$ cuts are described in sections $3.2 .1,3.2 .2$, and 3.3 respectively.

Three of the jets are required to have uncorrected $E_{T}>15 \mathrm{GeV}$, and be within $|\eta|<2$. The requirements on the fourth jet are looser, demanding uncorrected $E_{T}>8$ $\mathrm{GeV}$, and $|\eta|<2.4$. We only consider the four highest $E_{T}$ jets in the event. 


\subsection{Monte Carlo Simulation}

All of the Monte Carlo studies described in this section include a full detector simulation. We generate Monte Carlo $t \bar{t}$ events using the HERWIG program [32], with a top mass $m_{t}=175 \mathrm{GeV} / c^{2}$. We also use the ISAJET generator [34] as a cross-check. The expected $W+\mathrm{QCD}$ background is calculated using $W+3$ parton matrix elements in the VECBOS program [33]. The parton fragmentation process is simulated with a HERWIG shower module, which results in a model of $W+\geq 4$ jet production. Since these matrix elements are sensitive to the choice of mass scale in the strong coupling constant $\alpha_{s}$, we use two reasonable $Q^{2}$ scales, namely, the square of the average $P_{T}$ of the jets $\left(\left\langle P_{T}\right\rangle^{2}\right)$ and the square of the $W$ boson mass $\left(M_{W}^{2}\right)$.

We define a parton to match to a jet if the parton's direction is within $R<0.4$ of the jet centroid. If the parton matches to two or more jets, we choose the one with corrected $E_{T}$ nearest the parton $P_{T}$.

\subsection{Technique}

\subsubsection{Dijet masses}

The four jets in the event provide us with six dijet combinations, with only one (at most) corresponding to hadronic $W$ decay. In addition, the usual $W+$ QCD backgrounds are present.

To isolate the $W$ decay, one searches for jet pairs which have an invariant mass around $M_{W}$. Figure 4.1 shows the invariant mass resolution of dijet pairs which 
match in direction to quarks from hadronic $W^{\circ}$ decays in our Monte Carlo. All jet energies are corrected using the top-specific corrections discussed in section 3.1.2. and corrected as $W$ jets. When forming the dijet invariant mass, we may either take the mass of the quarks to be the cluster mass of the jet, or follow the example of the CDF top mass analysis[26] and use $0.5 \mathrm{GeV} / c^{2}$, the mass of light quarks. We investigated the effects of both choices on the resolutions of both quark $E_{T}$ and dijet mass. and found the resolutions to be nearly identical. So we decide to just conform to the top group's convention. A Gaussian fit to the peak gives a mean of $79.1 \mathrm{GeV} / c^{2}$ and a width of $11.7 \mathrm{GeV} / c^{2}$.

\subsection{2 $b$ tagging}

In order to reduce both the combinatoric and non-top background, we employ $b$ tagging, using both SECVTX and SLT tags. If we demand one tagged jet, then we have not only created a more top-enriched dataset, but we can reduce the number of dijet combinations from six to three. We may then demand a second tag, in order to assign a unique dijet pair to the $W$ decay. Since we already have a top-enriched sample, however, we need not be so strict in our requirements for the second $b$. Modest gains in tagging rates can be expected to produce large gains in double-tagging rates. We therefore employ a "loose" tagging method, based on the jet probability algorithm. 


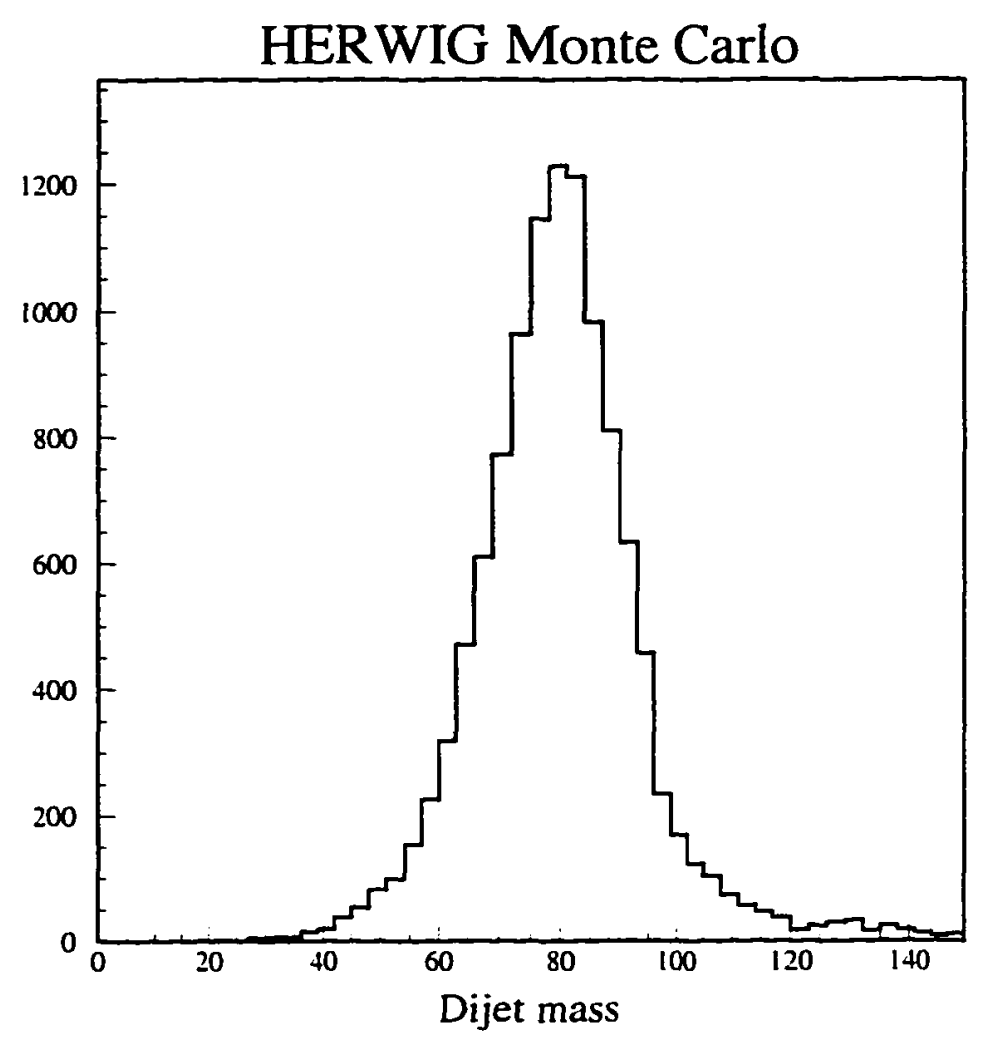

Figure 4.1: Dijet invariant mass from hadronic $W$ decay in $t \bar{t}$ : Monte Carlo.

\subsubsection{Loose $b$ tagging}

Even after applying SECVTX and SLT b-tagging to the $W+\geq 3$ jet sample, the jet probability algorithm finds additional bottom candidates (Fig 4.2). From this plot, it seems reasonable to tag jets with a jet probability of less than $5 \%$. Figure 4.3 shows the jet probability distribution in jets not tagged by SECVTX or SLT in $t \bar{t}$. Monte Carlo, further justifying this method and cut.

The $b$ jets tagged by jet probability but not by SECVTX mainly consist of jets 


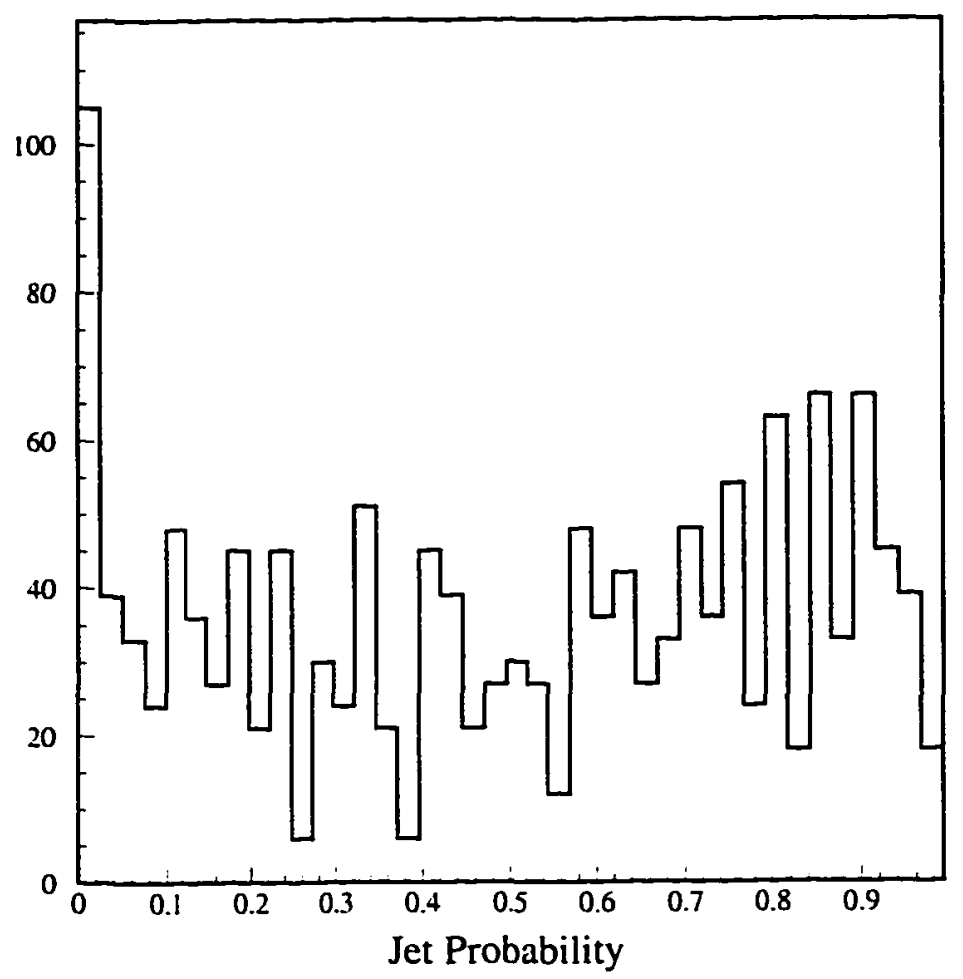

Figure 4.2: Jet probability of jets not tagged by SECVTX or SLT in $W+\geq 3$ jet events.

which have one or two good, significantly displaced tracks, as seen from Monte Carlo in figure 4.4.

\subsubsection{Triple-tagged events}

In this section, we investigate events with three tags, to determine if it would be possible to ignore one tag. For example, since SECVTX tags are the most reliable tags, we may expect that a clean sample of $W$ decays could come from ignoring a 


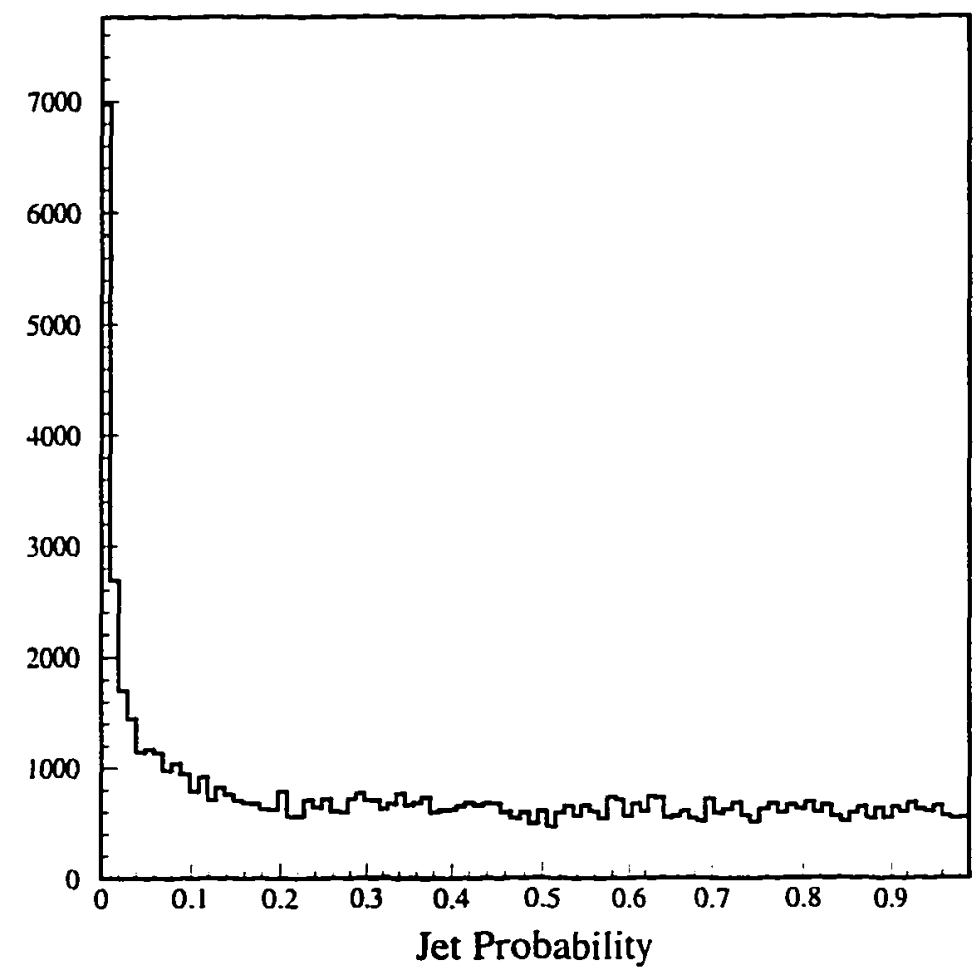

Figure 4.3: Jet probability of jets not tagged by SECVTX or SLT in $t \bar{t}$ Monte Carlo.

third JP or SLT tag. Also, if there are two JP tags, it may be reasonable to accept the lowest jet probability. The $W$ purities in triple-tagged Monte Carlo events are shown in table 4.1, where the uncertainties are statistical only. From it, we decide to accept only triple-tagged events with two SECVTX tags. We also note that the JP tags seem as reliable as the SLT tags. Also, for events with one SECVTX, one SLT, and one JP tag, we are as likely to find the $W$ by ignoring the SLT tag as by ignoring the JP tag. 

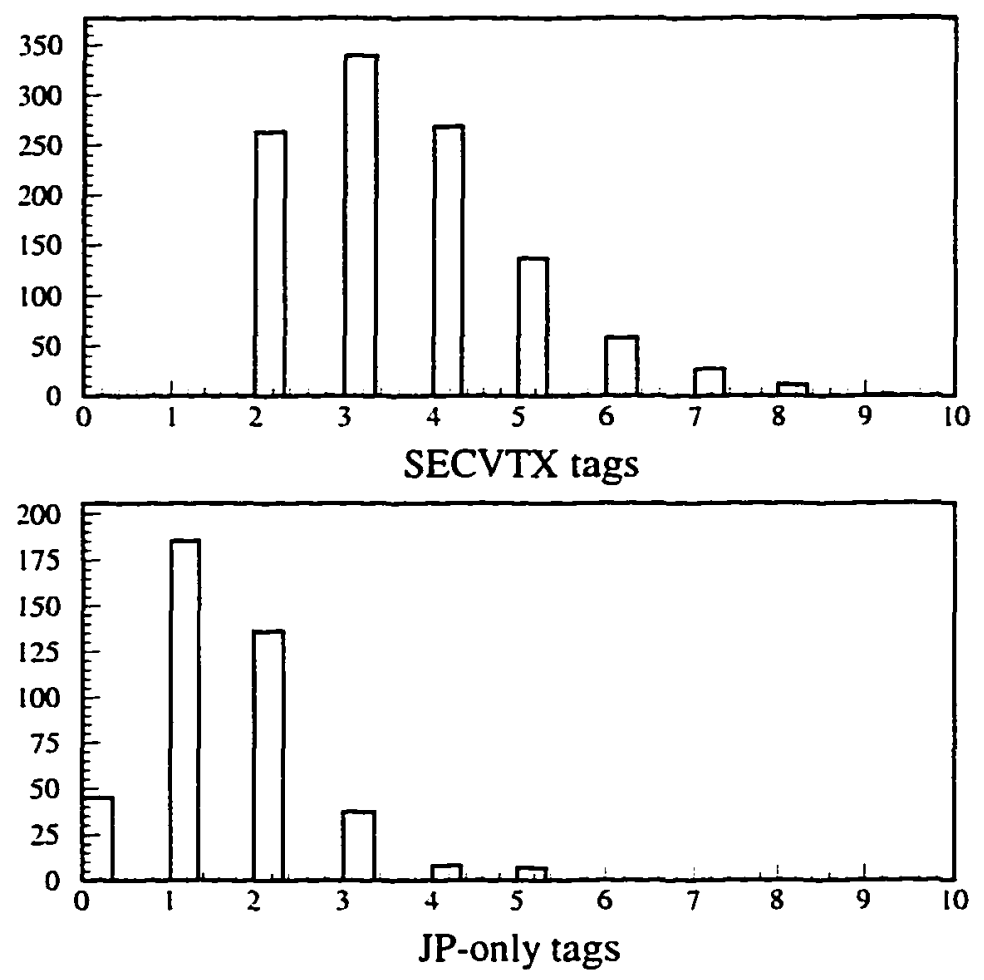

Figure 4.4: Number of tracks passing SECVTX pass 1 cuts with an impact parameter significance of three or more in $b$ jets tagged by SECVTX and JP.

\subsection{Monte Carlo Simulation Studies}

Figure 4.5 shows the dijet mass spectrum of the untagged jets for events with at least one SECVTX or SLT tag and a second tag, which could be SECVTX, SLT. or JP. The shaded region corresponds to the Monte Carlo process for which we are searching: jet pairs for which both jets match within $R<0.4$ to a generated quark from $W$ decay.

We see from figure 4.5 that this technique isolates a clear dijet mass excess around 


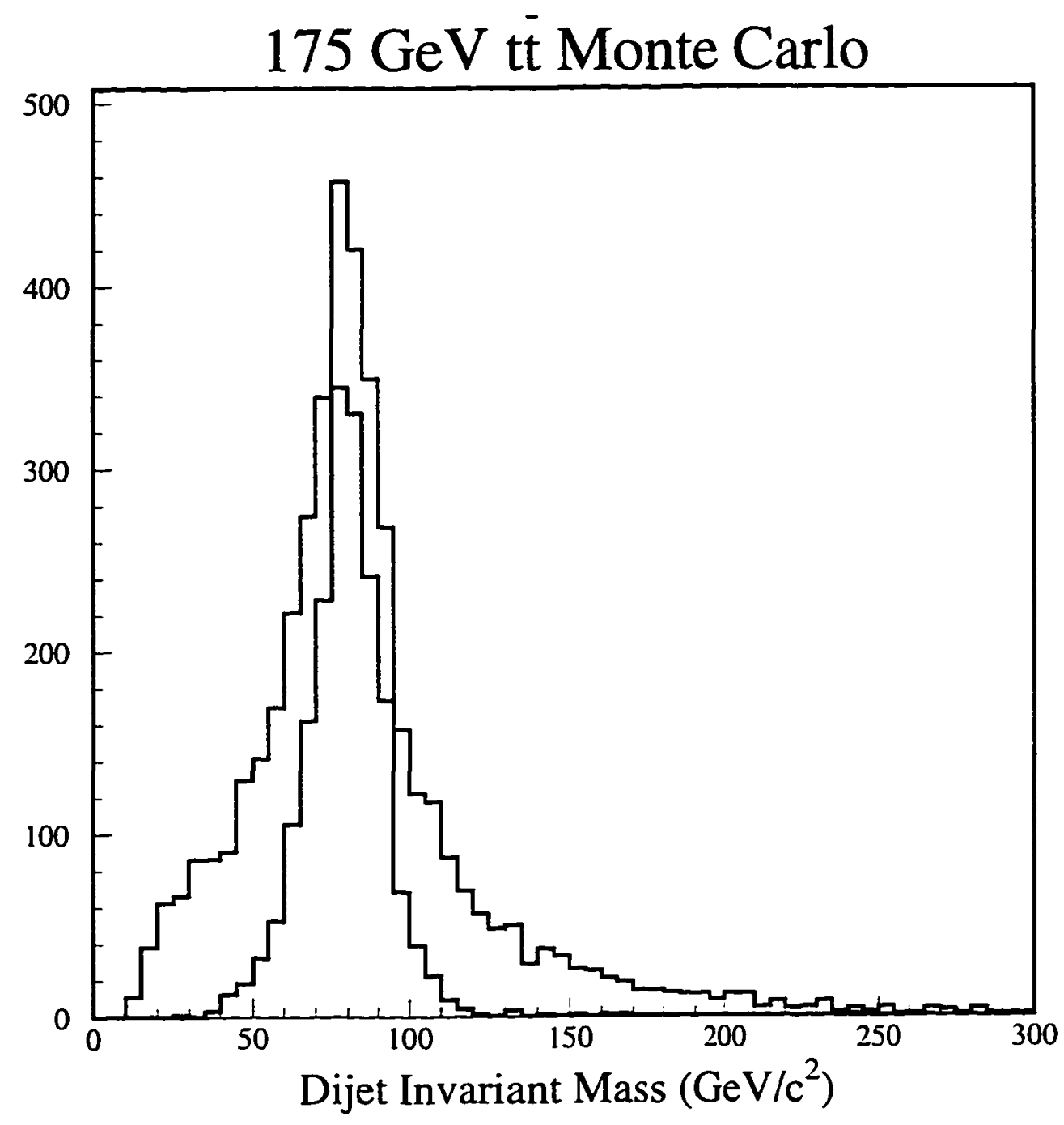

Figure 4.5: Dijet mass spectrum for the untagged jets in Monte Carlo for double $b$-tagged events. The shaded region corresponds to generated hadronic $W$ decay. 


\begin{tabular}{||c|c|c|c||}
\hline & \multicolumn{3}{|c||}{ Third tag } \\
\hline & SLT & JP & none \\
\hline SVX-SVX & $(45 \pm 4) \%$ & $(50 \pm 3) \%$ & $(52 \pm 1) \%$ \\
SVX-SLT & - & $(25 \pm 3) \%$ & $(38 \pm 2) \%$ \\
SLT-SLT & - & $(5 \pm 15) \%$ & $(35 \pm 3) \%$ \\
SLT-JP & - & $(15 \pm 6) \%$ & $(36 \pm 3) \%$ \\
SVX-JP & - & $(33 \pm 3) \%$ & $(43 \pm 1) \%$ \\
\hline
\end{tabular}

Table 4.1: The probabilities that the various forms of triple-tagged events correctly isolate the hadronic $W$ signal.

the $W$ mass. Allowing JP second tags increases the fraction of events that are doubletagged from $16 \%$ to $25 \%$, with only a small drop in signal purity. The technique finds the two correct $W$ jets in $43 \%$ of the double-tagged events. However, this number is sensitive to the amount of inital or final state radiation in the simulation, since the largest contamination ( $38 \%$ of double-tagged events) comes from events where the four highest $E_{T}$ jets do not correspond to two $b$ jets and two $W$ jets. We will hereafter define a gluon jet to be a jet which does not match within $R<0.4$ to a quark from $t \bar{t}$ decay. Other $t \bar{t}$ backgrounds include events where both $W$ 's decaved leptonically and only one lepton was identified (8\%), events where a $c$ quark from $I I$ decay was tagged (8\%), and mistags in $W$ jets $(3 \%)$.

Since a large background comes from events with gluon jets, we study whether it would help to reject five-jet events. For both tight $\left(E_{T}>15 \mathrm{GeV},|\eta|<2.0\right)$ and loose 
$\left(E_{T}>8 \mathrm{GeV} .|\eta|<2.4\right.$ ) definitions of the fifth jet, we see that the signal-to-noise ratio improves, but the signal significance $S / \sqrt{N}$ goes down a small amount for the tight fifth-jet veto, and significantly for the loose fifth-jet veto. So we implement no fifth-jet veto now, but note that it may help in future datasets, which are limited less by statistics and more by systematic uncertainties.

\subsection{Backgrounds}

The overall background level we will use comes from the CDF top mass analysis [35]. A likelihood fit is used, along with the various tag rates from top and background processes, to determine the most likely mixture of $t \bar{t}$ and background in the $W+\geq 4$ jet sample. The result is that $107 \pm 9$ events of the 163 event sample are background. The $37 b$-tagged events are found to be $(25 \pm 4) \%$ background, and the 23 SECVTXtagged events are found to be (12 \pm 4$) \%$ background.

To calculate double $b$-tagged backgrounds, we first consider the following processes which can be expected to give pairs of heavy-flavor jets: single-top, $b \bar{b}, c \bar{c}, W b \bar{b}, W c \bar{c}$, $Z b \bar{b}$, and $Z c \bar{c}$. We calculate how many $b$-tagged and double $b$-tagged events are expected from these processes. The remaining expected $b$-tagged background events are not from sources with true heavy flavor pairs, but may still obtain double-tags through fake tags. We calculate this contribution by applying the fake-tagging rates for the various taggers to the other three jets in the events. 


\subsubsection{Fake tag rates}

The rate of SLT mistags is taken to be $1.5 \%$ per jet, from [30]. The rate of jet probability mistags is taken to be $5 \%$ per taggable jet. Whether or not a jet is SVXtaggable, however, is correlated within events. Events with small longitudinal boosts, and events with a primary vertex $z$ position in the center of the SVX, are more likely to give taggable jets. To account for this, we separate the taggable fraction according to whether or not the event already has an SECVTX tag. We consider events in the high $P_{T}$ lepton samples well out of the $W$ signal region, where the lepton isolation is high $(I>0.2)$ or the $\not_{t}$ is low $\left(\not t_{t}<10 \mathrm{GeV}\right)$. We find that $(45 \pm 2) \%$ of jets in

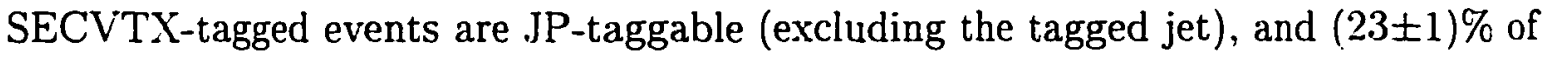
jets in events without SECVTX tags are taggable.

For the $b \bar{b}$ and $V b \bar{b}$ backgrounds, to improve double $b$-tagging statistics in the $W+\geq 4$ jet bin, we also include the rate of tags and double-tags in the $W+3$ jet bin, combined with the rate of fake-tagging a fourth jet.

\subsubsection{Single top backgrounds}

Single top quark processes, such as those shown in figure 4.6, are expected to be sources of $W$ bosons with two $b$ jets. The $W$-gluon fusion process is less likely to be tagged, since it tends to produce soft second $b$ jets. The cross-sections for these processes are calculated to be $(0.74 \pm 0.045) \mathrm{pb}$ for the process $W^{*} \rightarrow t b$ and $(1.44 \pm 0.43) \mathrm{pb}$ for the $W-g$ fusion process [37]. Acceptances and tag rates are then calculated from HERWIG and PYTHIA Monte Carlo. 
a)

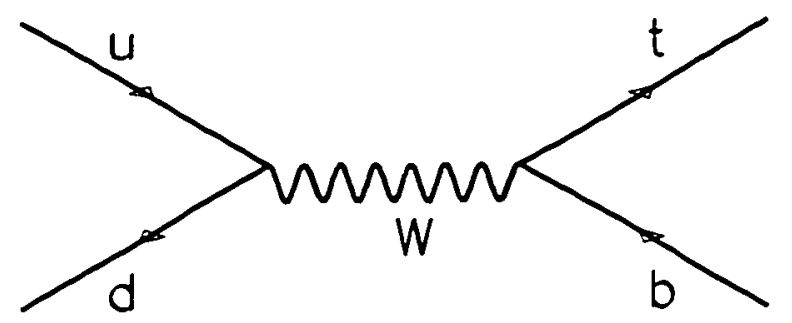

b)

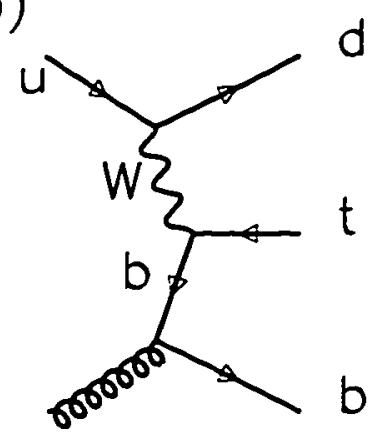

b)

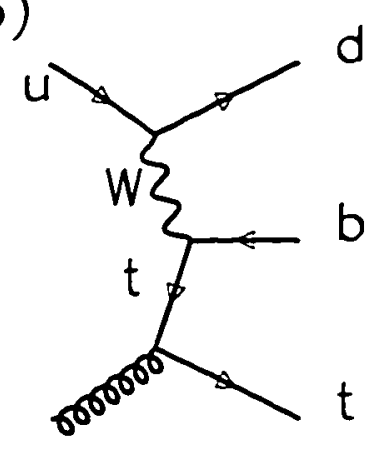

Figure 4.6: Single top quark production through a) virtual $\mathrm{W}$ and b) $W$-gluon fusion. 


\subsubsection{Non- $W^{*}$ background}

The non- $W$ background consists of leptons from $b \bar{b}$ and $c \bar{c}$ processes, along with fake leptons, photon conversions, $Z$, and Drell-Yan lepton pair processes. Studies of non$W$ background use a method which compares the lepton isolation and $Z_{t}$ in high $p_{T}$ lepton events to extrapolate from non- $W$ regions into the $W$ signal region. We divide the sample into four components:

- region $A: Z_{t}<15 \mathrm{GeV}$ and Isol $<0.1$

- region $\mathrm{B}: Z_{t}<15 \mathrm{GeV}$ and Isol $>0.2$

- region C: $Z_{t}>20 \mathrm{GeV}$ and $\mathrm{Isol}>0.2$

- region $\mathrm{D}: \not t_{t}>20 \mathrm{GeV}$ and $\mathrm{Isol}<0.1$ (W signal region)

If we assume that lepton isolation and $Z_{\iota}$ are uncorrelated for background events. the number of background events in the signal region $D$ is given by $D_{b g}=(A \times C) / B$. The fraction of non- $W$ events $F_{\text {non- } W}$ in the $W+\geq 3$ jet sample is calculated to be $10 \pm 4 \%$. The tagging rate for the low $\mathbb{Z}_{t}$ isolated leptons is then used to calculate backgrounds. This sample is measured to contain about $38 \% b \bar{b}$ events. To increase statistics for the double-tagging rates, we also include the rate from the sample of non-isolated leptons and large $Z_{t}$. 


\subsubsection{Wb $b \bar{b}, W c \bar{c}, Z b \bar{b}$, and $Z c \bar{c}$}

Heavy flavor pairs in $W+$ QCD events arise mainly due to the splitting of gluons into quark pairs. HERWIG and VECBOS Monte Carlos are used to model the probability for a $W+$ jets event to have either one or two heavy flavor jets [36]. $b \bar{b}$ pairs are expected in $3.4 \%$ of the $W+$ QCD events in our sample (before $b$-tagging), and $c \bar{c}$ pairs in $6.5 \%$. Tag rates are also derived from Monte Carlo.

We do the same for $Z+$ heavy flavor backgrounds, since we expect (from Monte Carlo) that $(7.4 \pm 2.3) \%$ of our $W+$ jets sample is actually misidentified $Z+$ jets.

\subsubsection{Final background estimate}

Once we know the number of tags, SVX tags, and double-tags from heavy flavor pair backgrounds, we consider the remaining expected background tags to come from either mistags, or processes with only one heavy flavor jet, such as $W W \rightarrow \ell \nu c \vec{s}$ or $s g \rightarrow W c$. These events can only pick up second tags through mistags. So we apply the mistag rates from section 4.5.1, which depend on whether or not the primary tag was an SVX tag, for each of the three remaining jets.

The final background estimates are given in table 4.2. The uncertainty on this estimate must account for various correlations. For example, errors on the Monte Carlo tag rates have a correlated effect on all the $V b \bar{b}$ and single top backgrounds. More importantly, estimating any background too high will be partially compensated by having fewer events subject to the mistagging rates described in the previous paragraph. We therefore use a toy . Monte Carlo technique to study the effect of 


\begin{tabular}{|c|c|c|c||}
\hline Process & SVX or SLT tags & SVX tags & Double b-tags \\
\hline Single top & $0.24 \pm 0.13$ & $0.16 \pm 0.09$ & $0.09 \pm 0.05$ \\
Non- $W$ & $0.75 \pm 0.52$ & $0.50 \pm 0.4$ & $0.12 \pm 0.12$ \\
$W b \bar{b}$ & $1.2 \pm 0.34$ & $0.90 \pm 0.27$ & $0.40 \pm 0.14$ \\
$W c \bar{c}$ & $1.0 \pm 0.28$ & $0.36 \pm 0.13$ & $0.08 \pm 0.03$ \\
$Z b \bar{b}, Z c \bar{c}$ & $0.25 \pm 0.095$ & $0.16 \pm 0.07$ & $0.06 \pm 0.03$ \\
Expected backgrounds & $9.2 \pm 1.5$ & $2.7 \pm 0.9$ & \\
Excess background & $5.8 \pm 1.6$ & $0.6 \pm 1.1$ & $0.6 \pm 0.2$ \\
\hline \multicolumn{3}{|c||}{ Final background estimate } & $1.3 \pm 0.3$ \\
\hline
\end{tabular}

Table 4.2: Estimates of non-t $\bar{t}$ double $b$-tagged backgrounds. The "Expected backgrounds" are the top mass analysis estimates, requiring only one tag, and the "Excess backgrounds" are obtained by subtracting the heavy-flavor-pair backgrounds from the total expected background.

varying all the input parameters by Gaussian uncertainties. The resulting background estimate for the double $b$-tagged sample is $1.3 \pm 0.3$ events.

\subsubsection{Background shape}

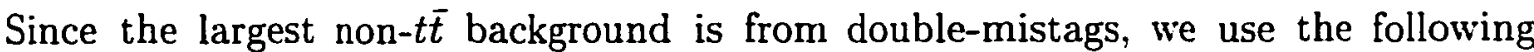
technique to estimate the dijet mass spectrum. We start from our $W+$ QCD Monte Carlo, and weight each dijet pair by the probability of mistagging the other two jets. The $W b \bar{b}$ and $W c \bar{c}$ event kinematics are seen to be similar to ordinary VECBOS, so they should be fairly well represented by this shape, too. The resulting histogram. 
shown in figure $4 . \bar{t}$, is only slightly different from the pretagged spectrum.

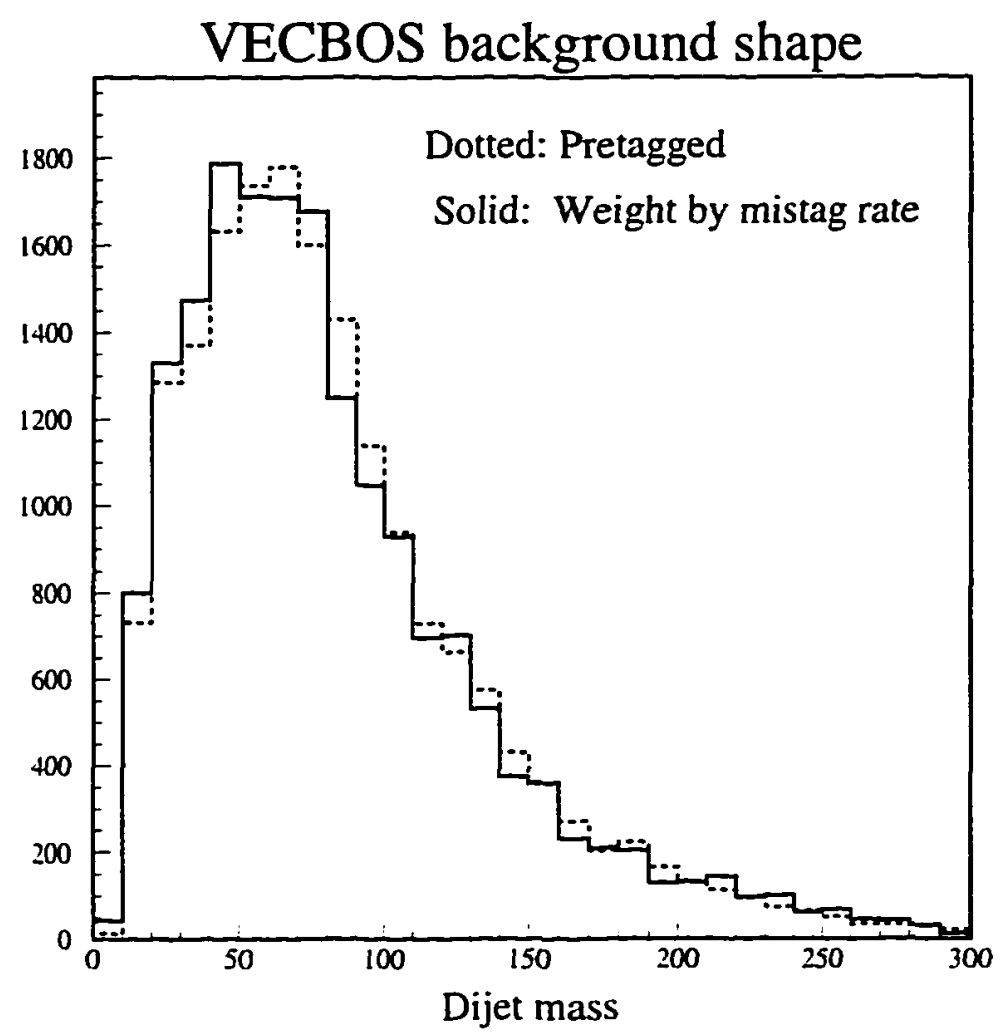

Figure 4.7: Comparison of the background histogram shapes with $Q^{2}=M_{11}^{2}$ before and after weighting entries by mistag probabilities.

\subsection{Results}

Figure 4.8 shows the dijet mass spectrum after demanding two $b$ tags, with at least one SECVTX or SLT, and possibly one loose jet probability tag, and excluding the $b$-tagged jets. Eleven events are found.

Eight of the eleven dijet combinations fall in the mass window of $60-100 \mathrm{GeV} / \mathrm{c}^{2}$. 
In $W+$ QCD . Ionte Carlo, only about a third of the dijet mass combinations fall in this window.

Of the eleven events, six have two "normal" $b$ tags (SECVTX or SLT), four enter with a jet probability tag, and one event has two SECVTX tags as well as a third JP tag. All of the JP-tagged events are used in the CDF top mass analysis (described in section 4.10.2), and the mass fitter assigned the loose-tagged jet as a $b$ in all cases except the high outlying event. The events are summarized in table 4.3 .

Figure 4.9 compares the dijet invariant mass in figure 4.8 obtained by excluding $b$-tagged jets with the dijet masses of the other five dijet combinations in these events.

In the top Monte Carlo sample, after applying the analysis cuts, $60 \%$ of the dijet combinations fell in the mass window of $60-100 \mathrm{GeV} / \mathrm{c}^{2}$.

The presence of a strong hadronic $W$ peak indicates that double-tagged $W+\geq 4$ jet sample is comprised largely of events with two $W$ 's and two $b$ 's. To test the quality of the leptonic $W$, we plot in figure 4.10 the lepton-neutrino transverse mass $M_{T}$, defined as $\sqrt{\left(E_{T \ell}+E_{T \nu}\right)^{2}-\left|\overrightarrow{P_{T \ell}}+\overrightarrow{P_{T \nu}}\right|^{2}}$, for the events in the mass window. The result is consistent with leptonic $W$ production.

\subsection{Notable Events}

Some of the double b-tagged events deserve further scrutiny.

The event at low dijet mass, Run 66573, Event 107219, appears to have a second 


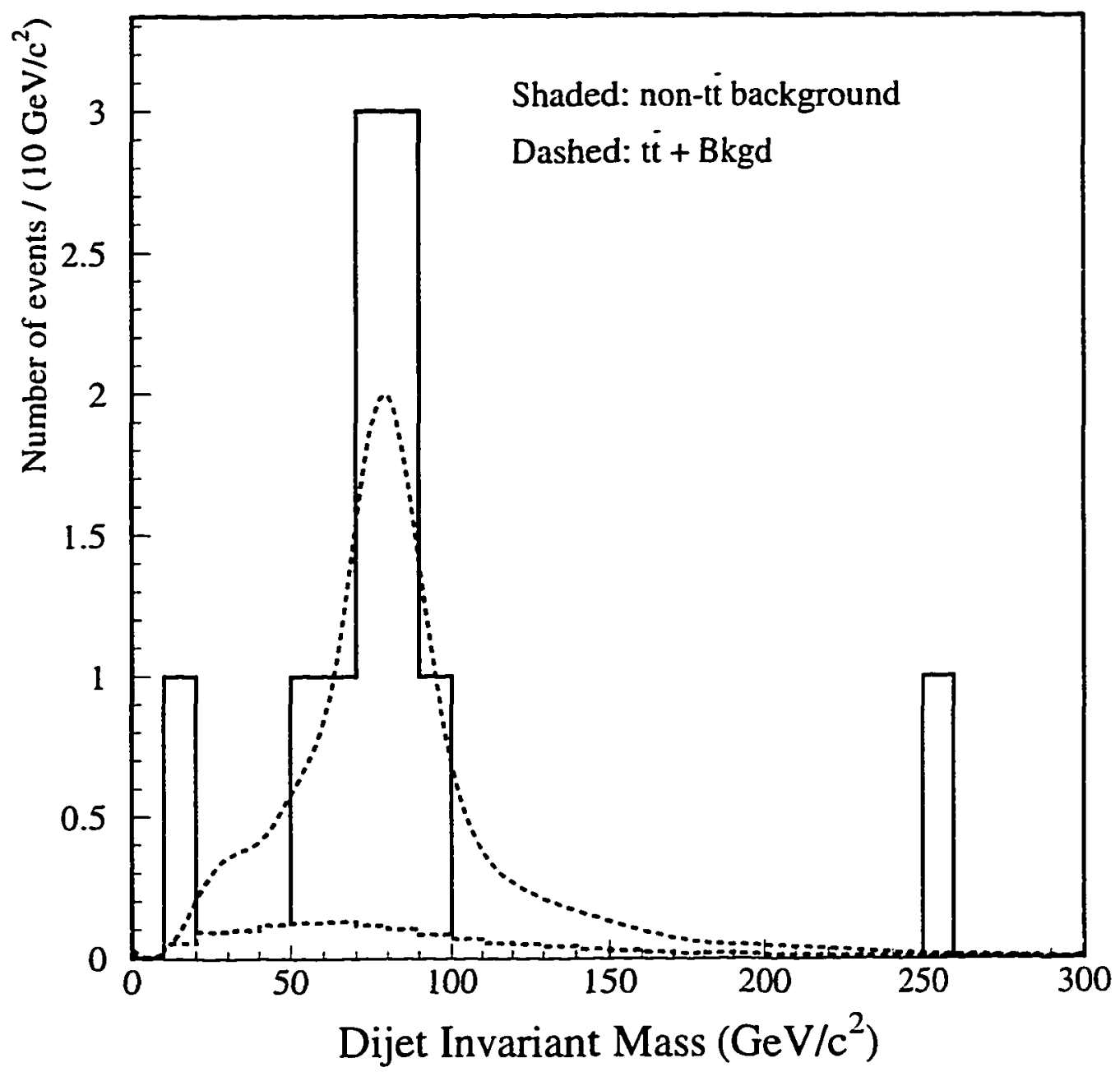

Figure 4.8: Dijet mass spectrum after demanding at least two $b$ tags, one of which must be SECVTX or SLT. 
Invariant masses in events with a second, loose tag
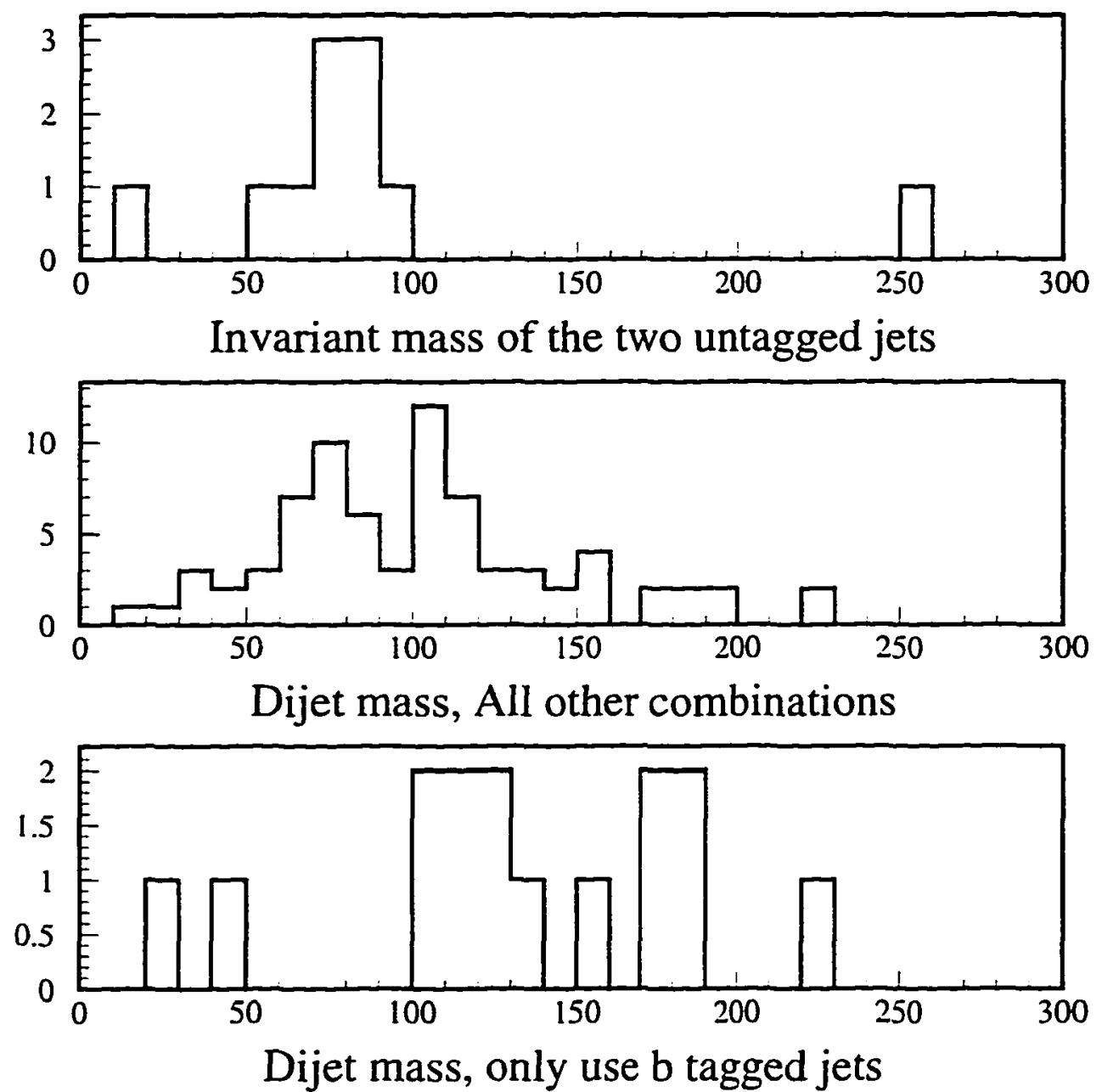

Figure 4.9: Comparison of dijet masses excluding loose $b$-tags with the other five combinations in the event which involve $b$-tagged jets, and the pairs of $b$-tagged jets. All jets are corrected as $W$ jets. 


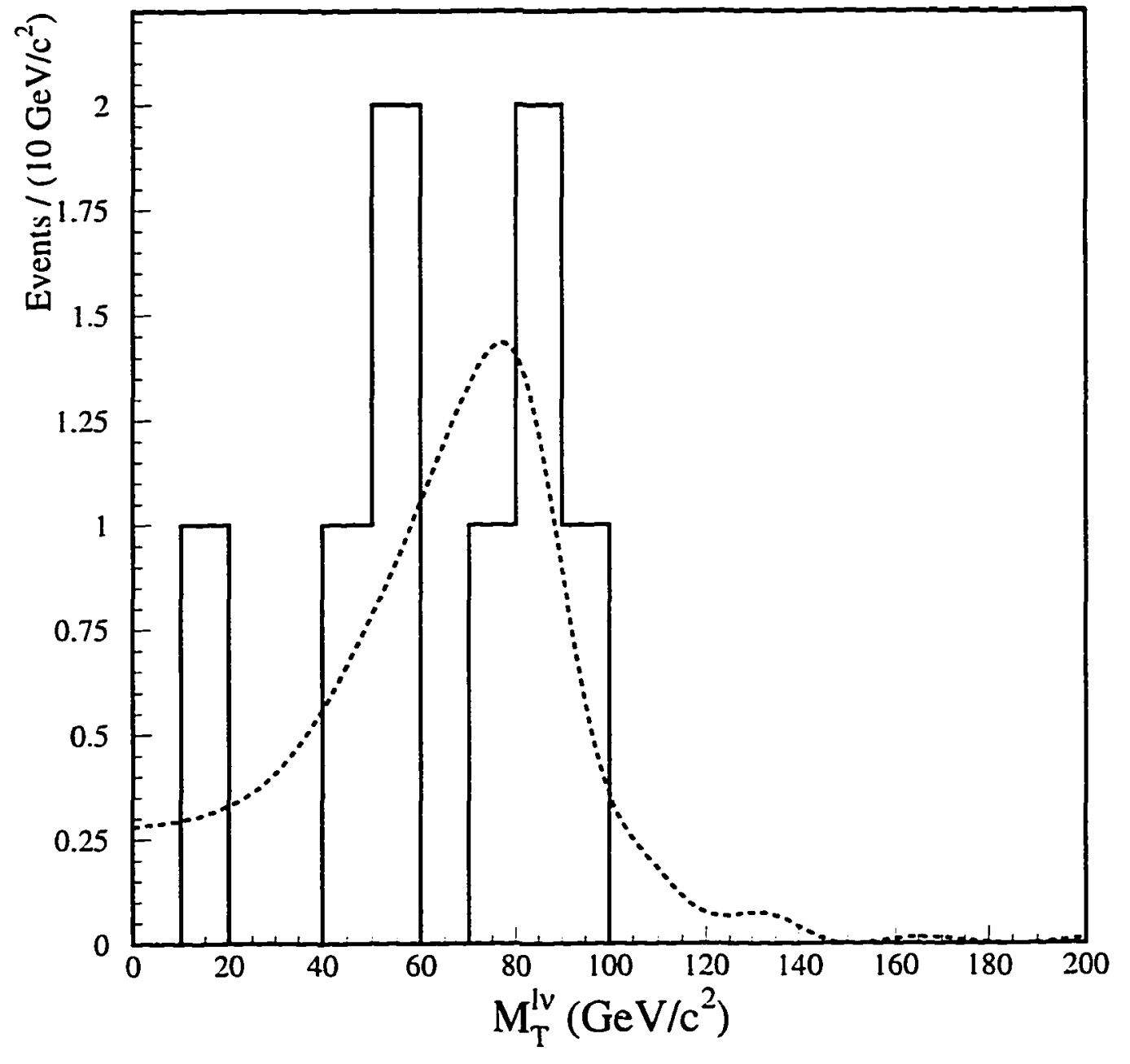

Figure 4.10: The lepton-neutrino transverse mass for the eight events in the hadronic $W$ mass window $60-100 \mathrm{GeV} / c^{2}$, along with Monte Carlo expectation for $t \bar{t}$ events. 


\begin{tabular}{||c|c|c|c|c|c||}
\hline Run & Event & lepton & $M_{T}\left(\mathrm{GeV} / c^{2}\right)$ & Tags & $M_{j j}\left(\mathrm{GeV} / c^{2}\right)$ \\
\hline 40758 & 44414 & $e$ & 62.0 & 2 SECVTX & 79.9 \\
43351 & 266423 & $\mu$ & 90.7 & SLT, loose JP & 255.5 \\
59698 & 31639 & $e$ & 95.4 & SECVTX, loose JP & 78.7 \\
63247 & 65096 & $e$ & 87.2 & SECVTX, loose JP & 80.2 \\
64721 & 229200 & $\mu$ & 11.1 & 2 SLT & 81.2 \\
65298 & 747402 & $\mu$ & 88.8 & SLT, loose JP & 59.2 \\
65581 & 322592 & $e$ & 56.7 & SECVTX,SEC+SLT & 65.2 \\
66573 & 107219 & $e$ & 40.7 & SECVTX,SLT & 18.2 \\
67824 & 281883 & $e$ & 58.2 & 2 SECVTX (loose JP) & 80.2 \\
67971 & 55023 & $\mu$ & 74.0 & 2 SECVTX & 97.2 \\
68464 & 547303 & $e$ & 40.9 & 2 SECVTX & 86.3 \\
\hline
\end{tabular}

Table 4.3: Double-tagged events and their hadronic $W$ mass and the transverse masses of the leptonic $W$ candidates. 
electron of $E_{T}=15.3 \mathrm{GeV}$ near the first one $(R=0.46)$, which fails only the fiducial and isolation cuts, and thus is counted as a jet. The two jets remaining after tagging are the "second electron" and a very nearby jet $(R=0.54)$. This event is shown in figure 4.11 .

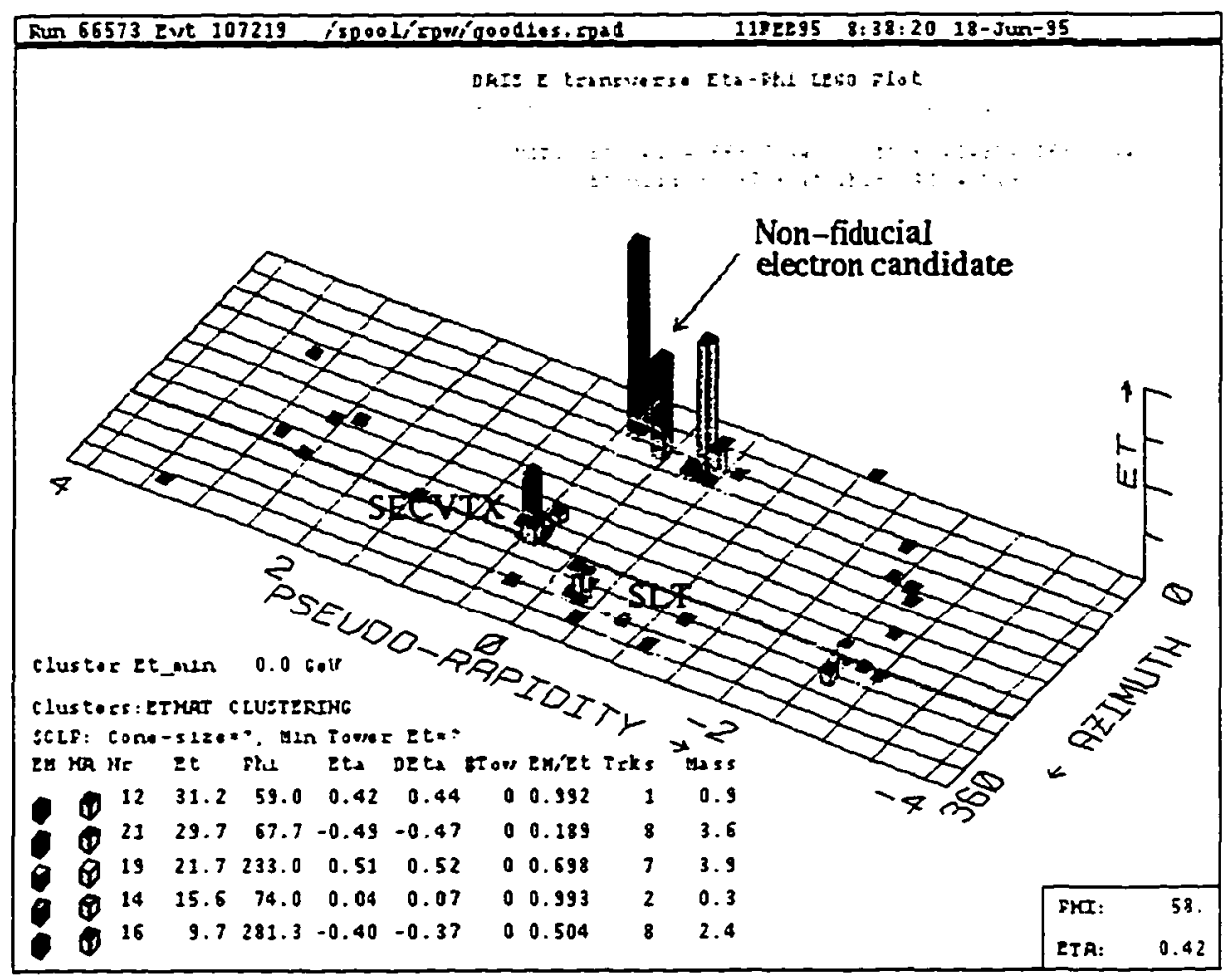

Figure 4.11: The low dijet-mass event: Run 66573, Event 107219.

The event with a high dijet mass is from Run 1A, Run 43351, Event 266423 . This muon event is shown in figure 4.12 . The third jet has a jet probability of $2 \%$, and the fourth jet has an SLT muon with a $P_{T}$ of only $2.1 \mathrm{GeV} / c^{2}$.

A hadronic tau candidate, with only one track, makes up one of the untagged jets in the double-SLT-tagged muon event, Run 64721, Event 229200. We keep it. because other CDF top analyses do not reject tau candidates. 


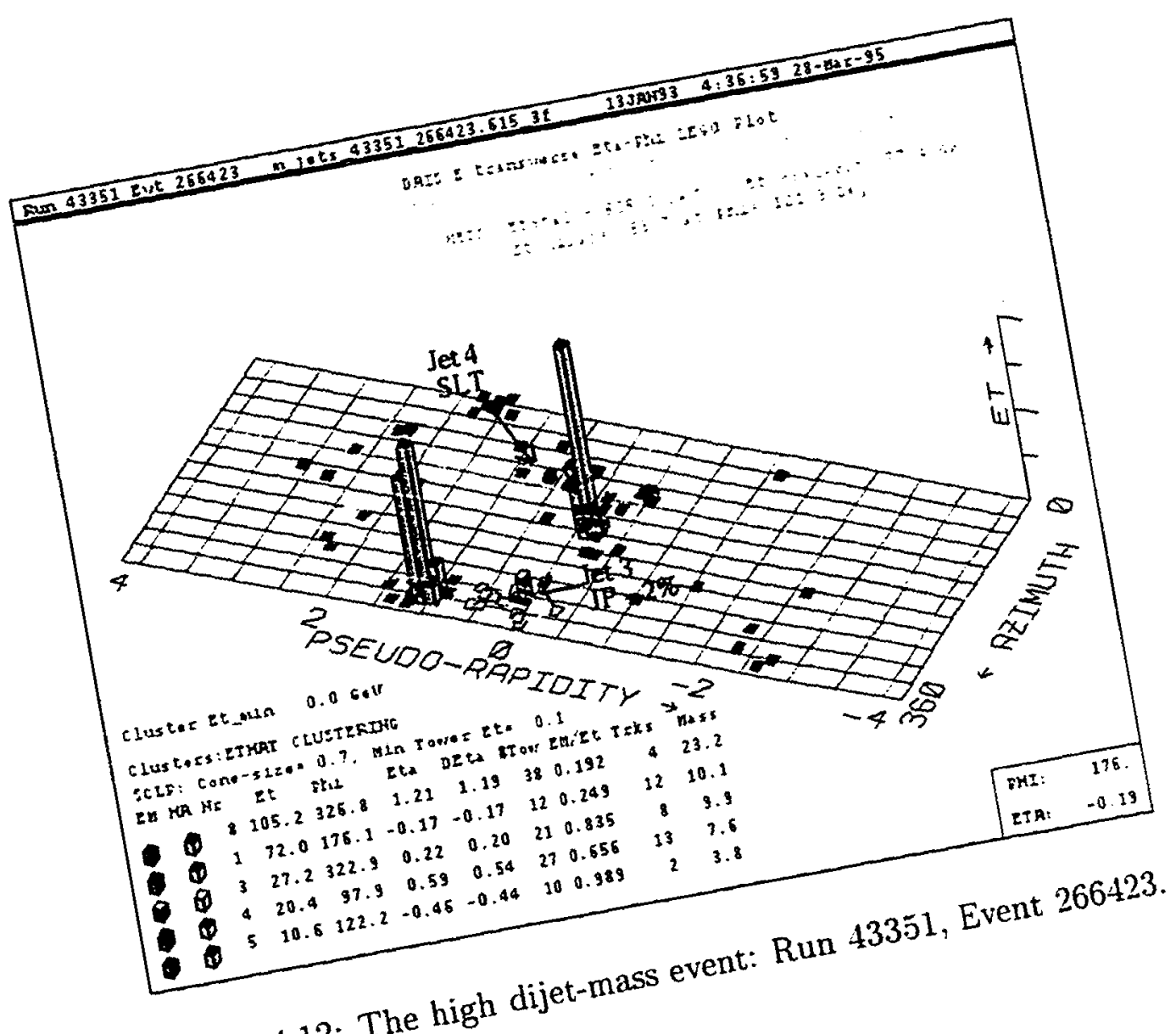

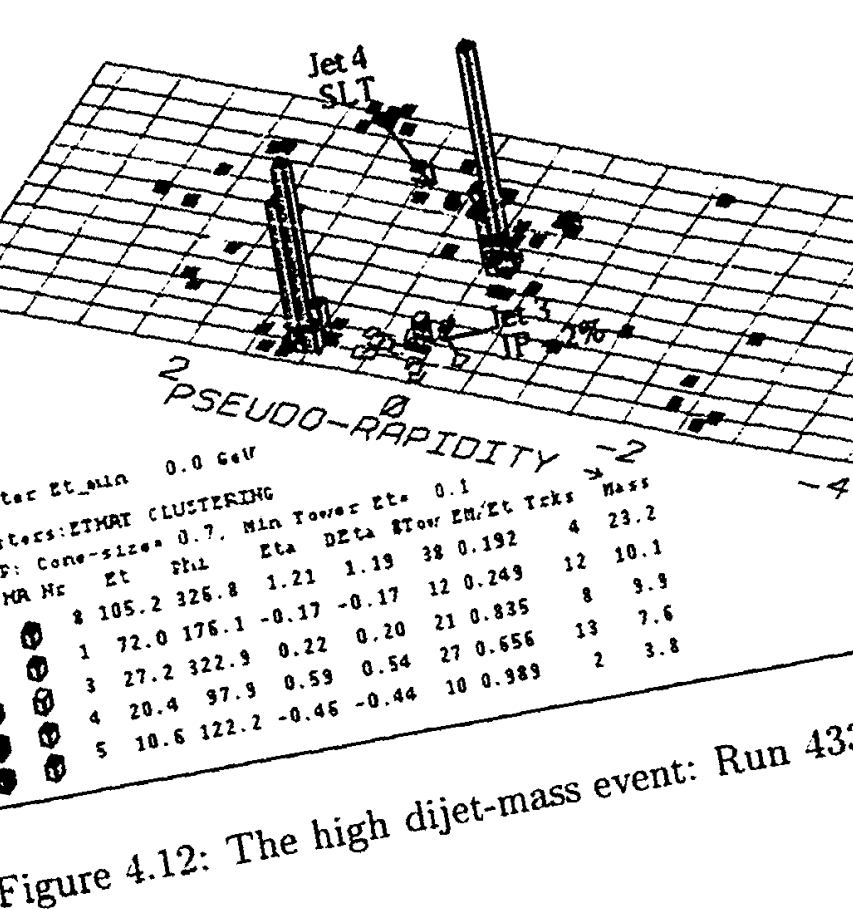




\subsection{Hadronic II Mass Fitting}

In this section, we discuss the maximum likelihood method used to extract the $I^{\circ}$ mass from the hadronic decay mode. We will fit to the following four parameters:

- $N_{b}$, the number of non- $t \bar{t}$ background events,

- $N_{c}$, the number of combinatoric background events,

- $N_{W}$, the number of hadronic $W$ 's, and

- $M_{W}$, the measured $W$ mass.

where $N_{b}+N_{c}+N_{W}$ is constrained to exactly the number of observed events.

The shape of the hadronic $W$ signal will be represented by a Gaussian of width 11.7 $\mathrm{GeV} / c^{2}$, the width of the hadronic $W$ peak in our Monte Carlo. Variations on the width will be assigned as a systematic uncertainty. The non-t $\bar{t}$ backgrounds will be represented by the fake-tagged histogram in figure 4.7. The top combinatorics histogram is made with Monte Carlo and requires double $b$ tagging, and also requires that both jets do not match within a cone of 0.4 to a generated quark from $W^{-}$decay.

All background histograms have both jets corrected as $W$ jets.

\subsubsection{Background term}

Since we have a calculated background of $1.3 \pm 0.3$ events, we may use it to constrain $N_{b}$, within Poisson statistics. Since $N_{b}$ is not an integer, we use a gamma function to replace the factorial in the Poisson distribution. The statistical variation is larger 

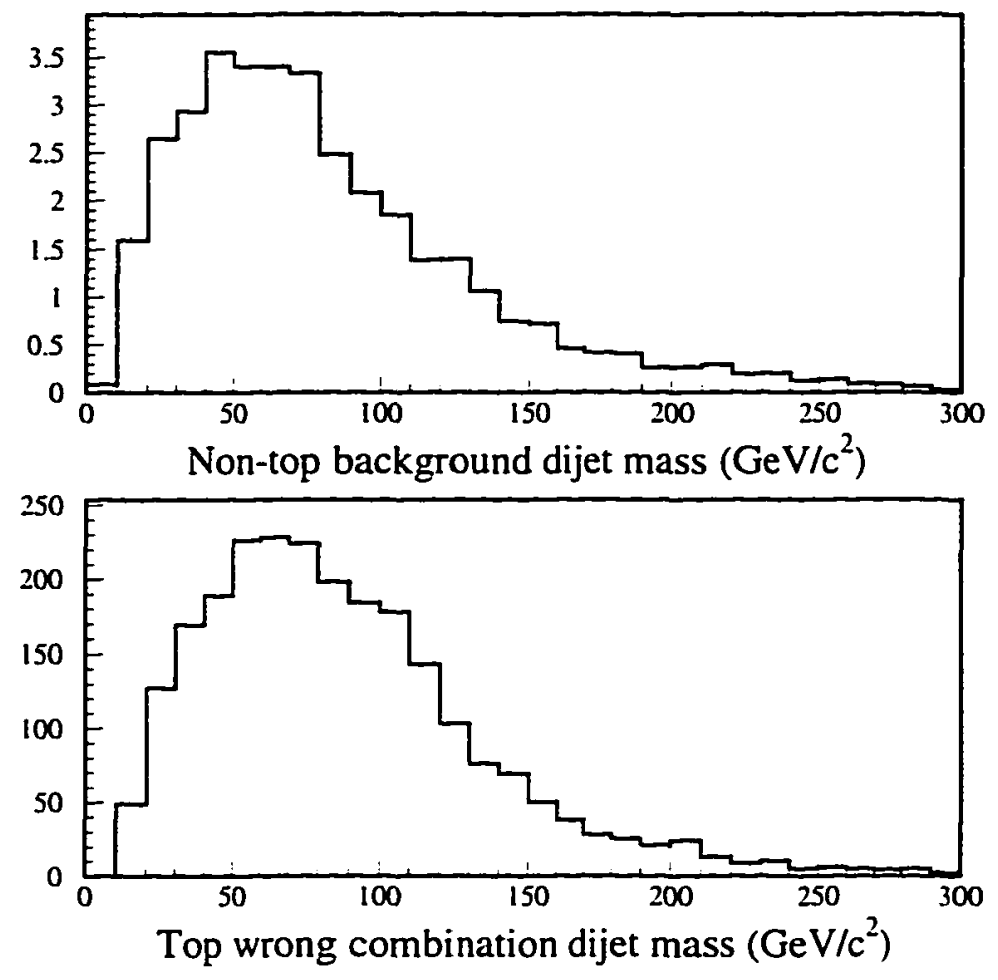

Figure 4.13: Background shapes used for a) non-t $\bar{t}$ background and b) top combinatorics.

than the uncertainty on the mean, so we can save variations on the mean background level as a systematic uncertainty.

\subsubsection{Constraining $N_{W}$ and $N_{c}$}

If we now proceed with only the background constraint term, the fit results are as follows:

$$
M_{\mathrm{IV}}=78.1 \pm 4.4 \mathrm{GeV} / \mathrm{c}^{2}
$$




$$
V_{b}=1.6 \pm 1.4
$$

$$
N_{W}=8.7 \pm 2.3
$$

While having 8.7 events assigned to be $W$ 's gives a low statistical uncertainty on the $W$ mass, it is inconsistent with our expectation from HERWIG that only $43 \%$ of entries from top are hadronic $W$ signal. Reality may be better modeled by putting in a constraint between $N_{W}$ and $N_{c}$ based on binomial statistics.

But before we implement this constraint, we must determine whether this $I^{\circ}$ fraction $f_{W}$ is known with a larger or smaller uncertainty than the expected binomial spread.

Since the largest contribution to combinatoric background comes events with gluon radiation among the four leading jets, we expect the largest contribution to the uncertainty on $f_{W}$ to come from this source as well. To study this, we first look at the double-tagging rates in $t \bar{t}$.Monte Carlo events with and without one or more gluon jets, summarized in table 4.4. From this information, we can construct a function which relates the purity of the hadronic $W$ signal $f_{W}$ to the amount of gluon radiation $f_{g}$, shown in figure 4.14 .

$$
f_{I V}=\frac{21.7\left(1-f_{g}\right)+1.9 f_{g}}{27.8\left(1-f_{g}\right)+23.2 f_{g}}
$$




\begin{tabular}{||c|c|c||}
\hline & no gluon & gluon \\
\hline \% double-tagged & $27.8 \%$ & $23.2 \%$ \\
$\%$ correct $W$ & $21.7 \%$ & $1.9 \%$ \\
\hline
\end{tabular}

Table 4.4: Double-tagging rates for events with and without one or more gluon jets.

In Monte Carlo, we see that $55 \%$ of $t \bar{t}$ events have at least one gluon jet. Since we have no way to confirm this number, we assume the possible gluon fractions have a flat distribution from zero to one, and we base our systematic uncertainty from hard gluons on varying the fraction of events with at least one gluon jet by one standard deviation, or $30 \%$, around a mean of $55 \%$. From the above information, we see that a change of $30 \%$ in the gluon fraction corresponds to a change of $20 \%$ in the purity of the $W$ signal from $t \bar{t}$ events.

Next, we ask what else can cause changes in the $W$ signal purity. To investigate the effects of changes in the tagging and mistagging rates, we vary the input tagging rates by one sigma in the simulation. The final signal purity only changes by a few percent at most, since the signal and background tagging rates go up or down together. The uncertainty on the $W$ purity is dominated by the contribution from hard gluon radiation.

So finally, we obtain an uncertainty on $f_{W}$ of $20 \%$. The spread of a binomial for $N=9.7$ and $p=0.43$ is given by $\sqrt{p(1-p) / N}$, or $16 \%$. Since neither of these effects dominates, we must include both in the likelihood by convoluting the binomial term 


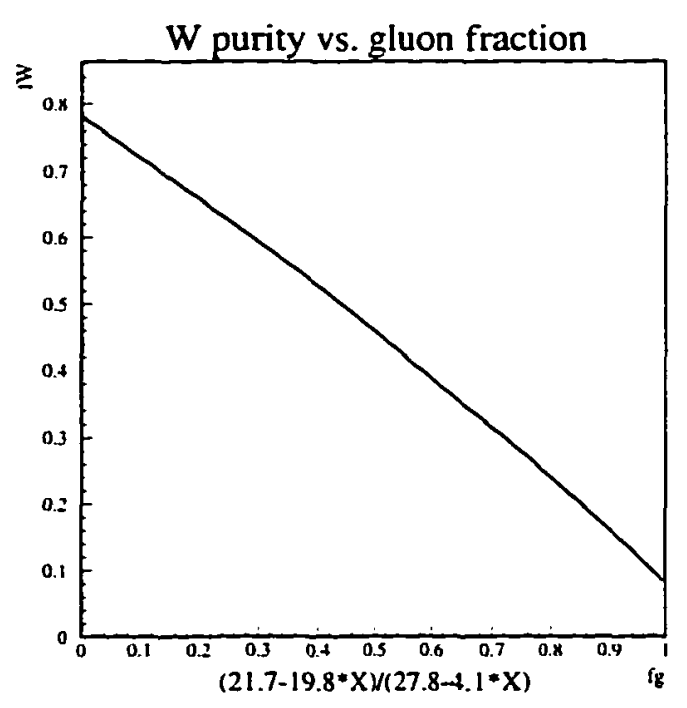

Figure 4.14: The relation between gluon fraction and $W$ purity for a double-tagged $t \bar{t}$ sample

with a Gaussian smearing. Again, we use gamma functions to interpolate between integer values for factorials. The term in the likelihood is then

$$
\int_{0}^{1} d p \frac{1}{\sqrt{2 \pi} \sigma} \exp \left(-\frac{\left(p-f_{W}\right)^{2}}{2 \sigma^{2}}\right) \frac{\Gamma\left(N_{W}+N_{c}+1\right)}{\Gamma\left(N_{W}+1\right) \Gamma\left(N_{c}+1\right)} p^{N_{W}}(1-p)^{N_{c}}
$$

The comparison of smeared and unsmeared shapes is given in figure 4.15 .

One concern about using this term is that since the Gaussian's tails extend beyond zero and $N$, the term will not be normalized with respect to variations in $N_{\text {top }}$. We only expect $N_{\text {top }}$ to vary by about one event, however, and this term stays normalized to within a percent under such variations. 


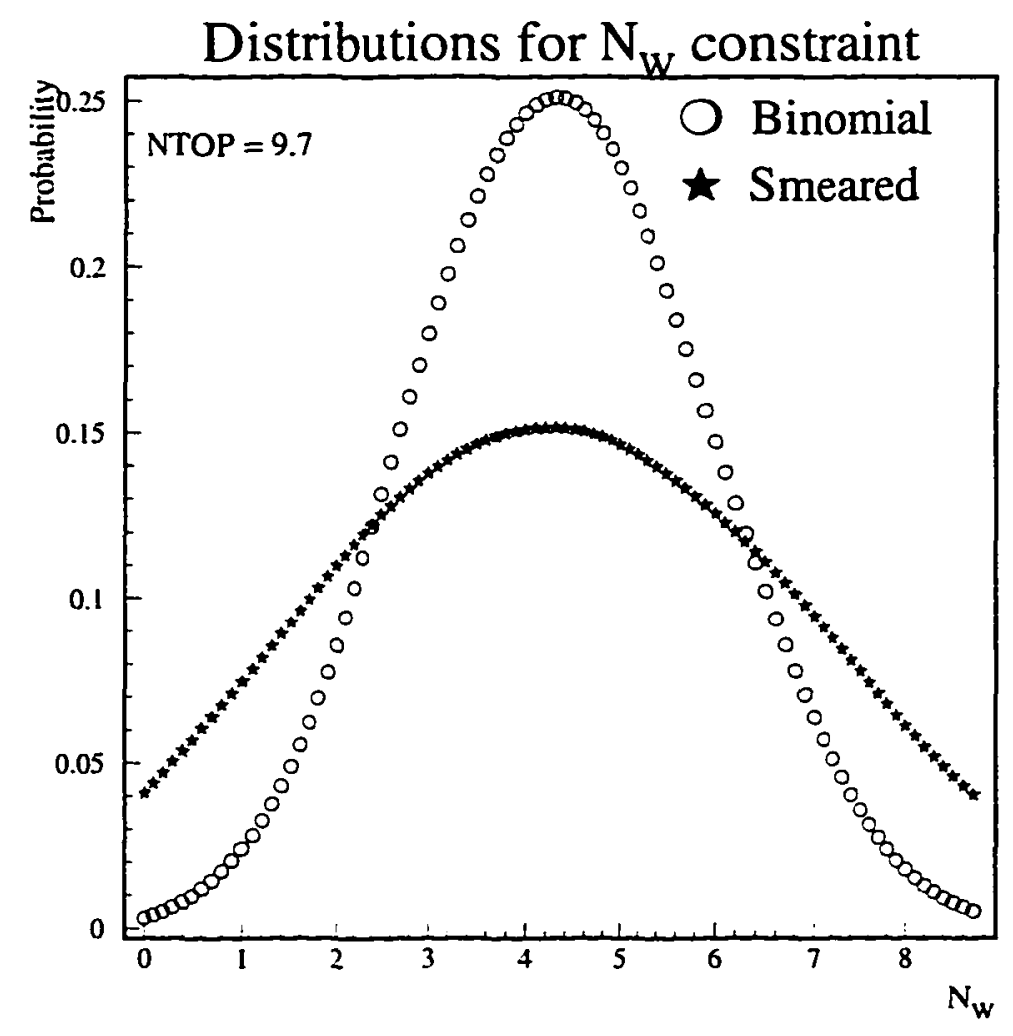

Figure 4.15: Comparison of the smeared and unsmeared binomial constraints on $N_{W}$.

\subsubsection{Comparison of likelihood functions}

The likelihood function, including background, smeared binomial, and shape terms, is now given by

$$
\mathcal{L}=P\left(N_{b}\right)\left(\int d p G(p) B\left(N_{W}, N_{c}, p\right)\right) \prod_{i=1}^{N} \frac{N_{b} f_{b}+N_{c} f_{c}+N_{W} G\left(M_{W}\right)}{N_{b}+N_{s}+N_{W}}
$$

where P, B, and G represent Poisson, binomial, and Gaussian functions.

To decide whether or not to use the smeared binomial constraint term, we first 
must see whether each technique gives reasonable errors. To check this. we run 1000 pseudo-experiments of eleven events each, where the input masses are chosen randomly from Monte Carlo. We fit each pseudo-experiment and plot the pull of the measured $W$ mass, the ratio of difference between the measured and true $W$ mass to the measured uncertainty. Pull distributions should be Gaussians centered at zero with unit width. Both techniques give good pull distributions, as shown in figure 4.16 , and both give similar errors on $M_{W}$. We finally decide to include the smeared binomial constraint.
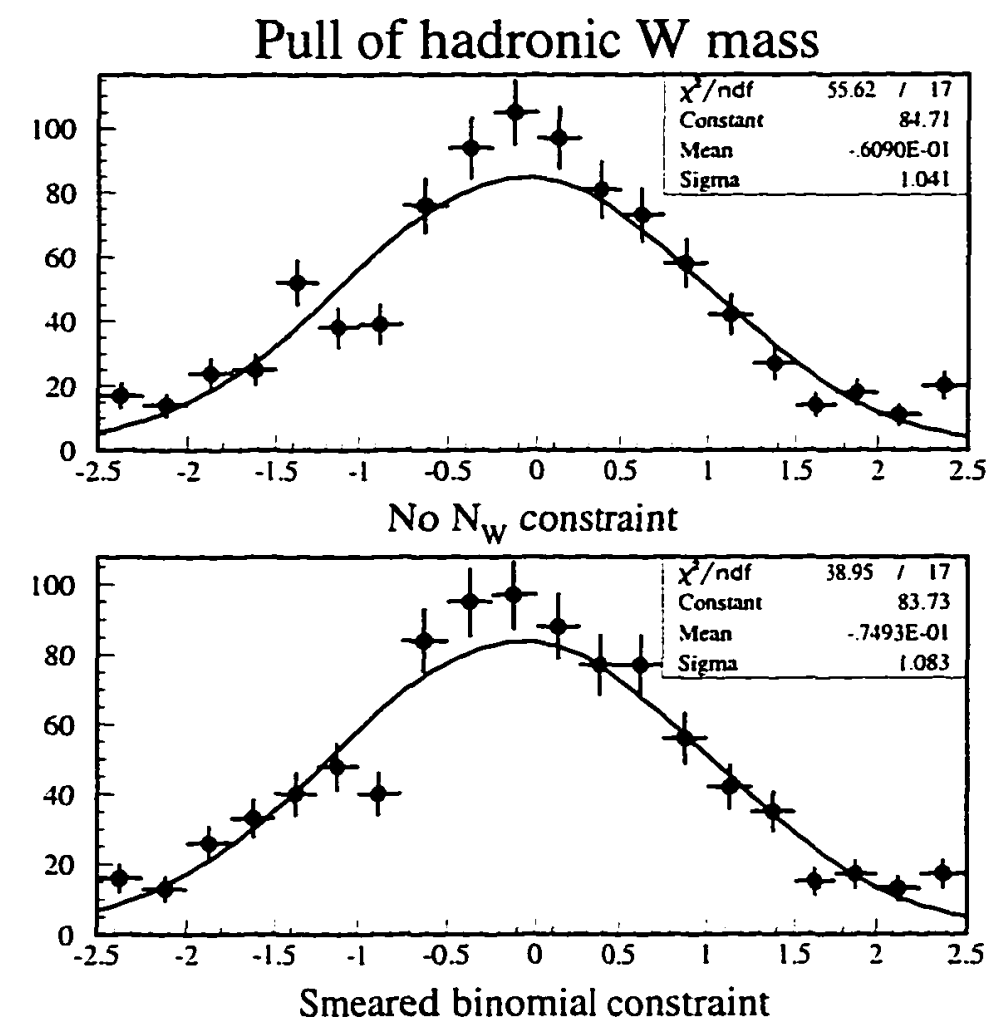

Figure 4.16: Pull distributions from 1000 pseudo-experiments of 11 events each. 
Applying this fit technique to our data, we get the following results:

$$
\begin{gathered}
M_{W}=78.3 \pm 5.1 \mathrm{GeV} / \mathrm{c}^{2} \\
N_{b}=1.2_{-1.1}^{+1.3} \\
N_{W}=6.4 \pm 2.0
\end{gathered}
$$

The statistical uncertainty on the $W$ mass is higher than before, because the fit allows fewer $W$ candidates. These results are compared to the pseudo-experiments in figure 4.17 .

\subsubsection{Systematic uncertainties}

The results of studies of systematic uncertainties are shown in table $4 . \overline{5}$. Some are done by refitting with different templates or fit techniques, and some use the median $W$ mass returned by an ensemble of 1000 pseudo-experiments of eleven events each.

\section{Non- $t \bar{t}$ background}

The background template used for the standard fit uses dijet combinations from a VECBOS sample using $Q^{2}=M_{W V}^{2}$. To study the effect of the $Q^{2}$ scale choice, we redo the fit using a template made with $Q^{2}=\left\langle P_{T}\right\rangle^{2}$. The shapes are compared in figure 4.18 . We also vary the expected background level by \pm 0.3 events. Since the overall background level is small, no effect from either variation is observed. 

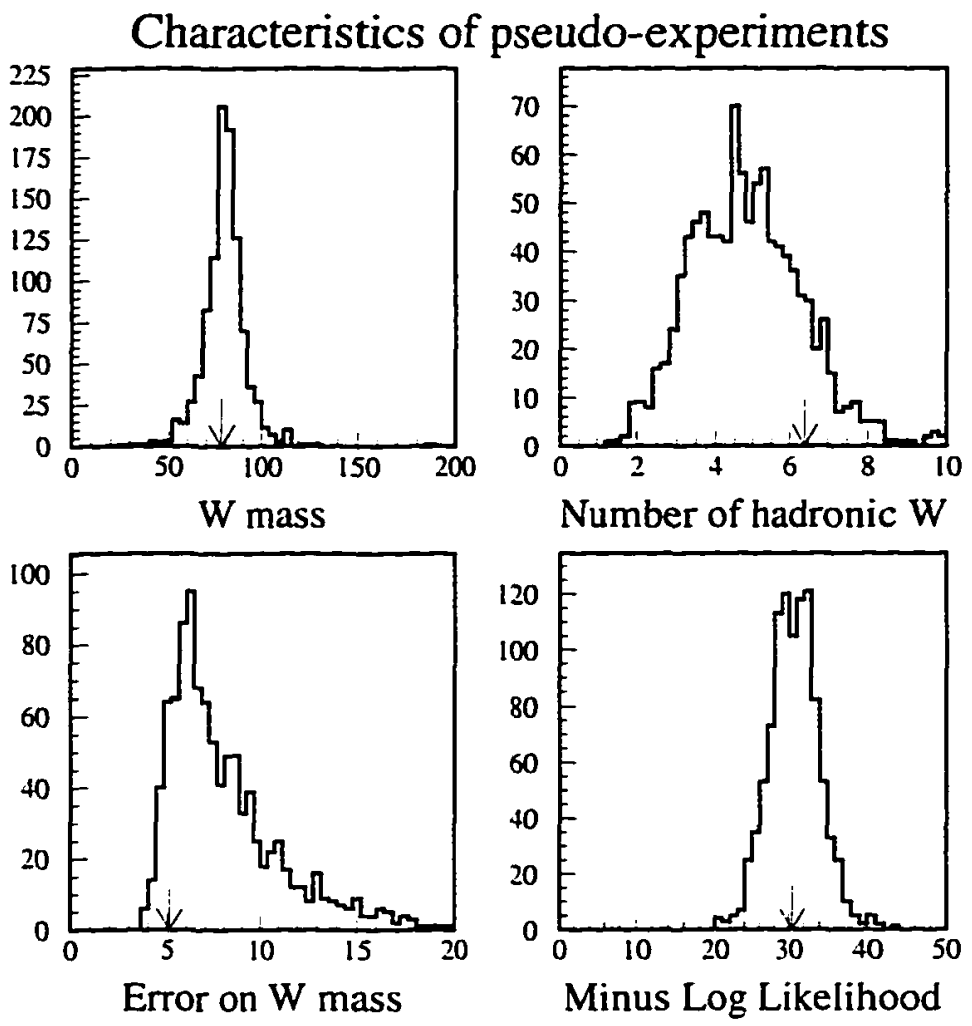

Figure 4.17: Distributions from 1000 pseudo-experiments of 11 events each. Arrows represent the value obtained fitting to the data.

\section{Top background}

Here, we refit with templates made from HERWIG Monte Carlo with top masses of 160 and $190 \mathrm{GeV} / \mathrm{c}^{2}$. Also, since we expect $80 \%$ of our background to come from hard gluon events, we refit with templates that have $70 \%$ and $90 \%$ gluon events. The net effect is less than $0.1 \mathrm{GeV} / c^{2}$.

Also, we refit using a $t \bar{t}$ background template from ISA.JET Monte Carlo with a top mass of $175 \mathrm{GeV} / \mathrm{c}^{2}$. No shift is observed. 


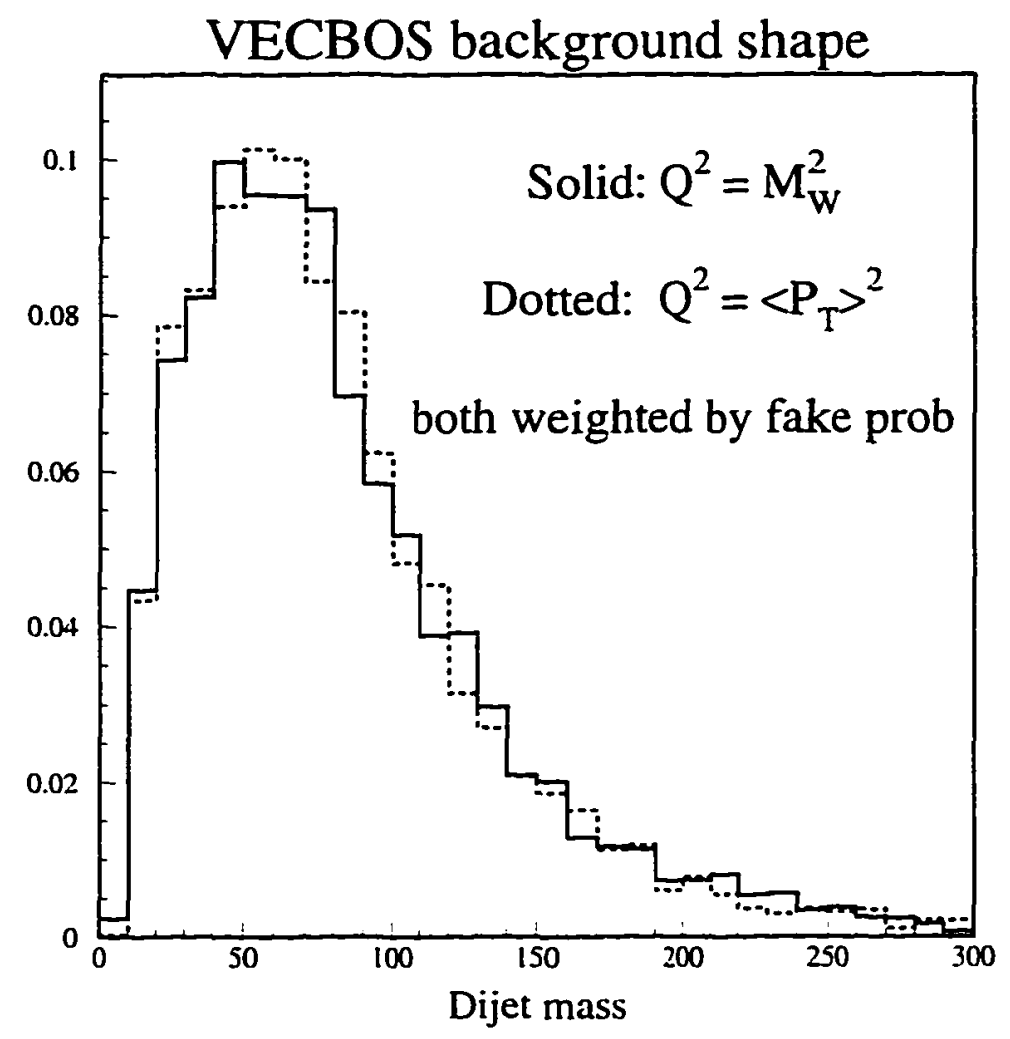

Figure 4.18: Comparison of the background histograms with $Q^{2}=M_{W}^{2}$ and $Q^{2}=P_{T}^{2}$.

\section{Fit method}

Many aspects of the likelihood could be varied. The constraints could be removed, or fixed and not allowed to vary. The total number of events could be allowed to fluctuate, with a Poisson constraint to $N_{\text {obs }}$. These variations shift the returned top mass by a maximum of $0.2 \mathrm{GeV} / c^{2}$, as shown in table 4.6. 


\section{Fitted $W$ width}

Here we use pseudo-experiments for variations, to avoid the statistical effects. Varying the jet energy resolution by $\pm 10 \%$ results in a variation of the reconstructed dijet mass of $10 \% / \sqrt{2}$, or $\pm 0.8 \mathrm{GeV} / c^{2}$. So we run pseudo-experiments where we fit to widths of 10.9 and $12.5 \mathrm{GeV} / c^{2}$ instead of $11.7 \mathrm{GeV} / c^{2}$, and look for variations in the median returned $W$ mass. The resulting uncertainty is $0.2 \mathrm{GeV} / c^{2}$.

\section{Sideband events}

Since those two sideband events are both far out on the tails of the distribution, we check the result of throwing out each of the events and refitting. This results in an uncertainty of $0.1 \mathrm{GeV} / c^{2}$.

\section{Monte Carlo statistics}

To estimate this, we perform 1000 experiments using the data events but in which the number of events in each bin in the background templates is allowed to fluctuate within Poisson statistics. The returned $W$ mass has a fitted width of $0.5 \mathrm{GeV} / c^{2}$.

\section{Jet energy scale}

Next, we determine the systematic uncertainties on how well the measured jet energies correspond to the original quark energies. This uncertainty can be broken into three components: uncertainty on the calorimeter response to incident particles, uncertainty on the Monte Carlo quark fragmentation process, and uncertainty on 


\begin{tabular}{|c|c|c|}
\hline Quantity varied & $M_{W} \operatorname{Result}\left(\mathrm{GeV} / c^{2}\right)$ & Sys. unc. \\
\hline \multicolumn{3}{|l|}{ Non- $t \bar{t}$ background } \\
\hline constr. to $1.6 \mathrm{ev}$ & $78.3 \pm 5.1$ & 0.0 \\
\hline constr. to $1.0 \mathrm{ev}$ & $78.3 \pm 5.1$ & \\
\hline$Q^{2}=\left\langle P_{T}\right\rangle^{2}$ & $78.3 \pm 5.1$ & 0.0 \\
\hline \multicolumn{3}{|l|}{ Top background } \\
\hline$m_{t}=160 \mathrm{GeV}$ & $78.3 \pm 5.2$ & 0.1 \\
\hline$m_{t}=190 \mathrm{GeV}$ & $78.2 \pm 5.0$ & \\
\hline ISAJET, $m_{t}=175$ & $78.2 \pm 4.8$ & 0.1 \\
\hline $70 \%$ hard gluon & $78.3 \pm 5.1$ & 0.1 \\
\hline $90 \%$ hard gluon & $78.4 \pm 5.1$ & \\
\hline \multicolumn{3}{|c|}{ Fitted width (pseudo-experiments) } \\
\hline $10.9 \mathrm{GeV}$ & median 79.8 & 0.2 \\
\hline $12.5 \mathrm{GeV}$ & median 79.4 & \\
\hline \multicolumn{3}{|l|}{ Sideband events } \\
\hline Throw out low one & $78.2 \pm 4.1$ & 0.1 \\
\hline Throw out high one & $78.2 \pm 4.2$ & \\
\hline
\end{tabular}

Table 4.5: Systematic uncertainties on the $W$ mass measurement due to fit techniques. 


\begin{tabular}{||c|c|c|c||}
\hline \multicolumn{2}{||c|}{ Fit technique } & \multicolumn{2}{c||}{ Total syst. unc.: $0.2 \mathrm{GeV} / c^{2}$} \\
\hline$N_{b}$ constr? & $N$ constr? & $N_{W}$ constr? & $N_{W}\left(\mathrm{GeV} / c^{2}\right)$ \\
\hline Poisson & hard & none & $78.1 \pm 4.4$ \\
Poisson & hard & smeared binomial & $78.3 \pm 5.1$ \\
hard & hard & none & $78.1 \pm 4.4$ \\
none & hard & none & $78.4 \pm 4.9$ \\
Poisson & hard & binomial & $78.4 \pm 5.5$ \\
Poisson & Poisson & smeared binomial & $78.3 \pm 5.1$ \\
Poisson & Poisson & hard & $78.5 \pm 6.0$ \\
Gaussian & hard & Gaussian & $78.4 \pm 5.4$ \\
\hline
\end{tabular}

Table 4.6: Systematic uncertainties on the $W$ mass measurement due to different background constraints. 
the amount of energy collected in the jet cone from the underlying $p \bar{p}$ interaction or from other interactions in the same beam crossing,. For all of these, we use techniques developed for the CDF top mass analysis [12]. We apply shifts (up or down) to our Monte Carlo jet energies, and then use these shifted datasets as inputs to pseudo-experiments and refit them against the unshifted templates. The shift in the resulting median $W$ mass of all the pseudo-experiments is taken to be the systematic uncertainty.

We first include a shift of $\pm 1 \%$ to the raw jet energies representing detector stability. The uncertainty on the detector response to individual particles has been studied in Ref. [38]. The uncertainty is about $2.5 \%$ with a slight asymmetry and $E_{T}$-dependence, applied after the absolute energy scale corrections and before any out-of-cone or top-specific corrections. Finally, the uncertainty on the correction for multiple interactions is taken to be $30 \%$ of the correction. These three effects are added in quadrature to form the calorimeter effects uncertainty.

In the top mass analysis, the difference between data and Monte Carlo modeling of soft gluon radiation was measured by looking at $E_{T}$ flow in samples of $W, Z$, and $\gamma$ bosons with single jets. The fraction of the energy in the annular region $0.4<R<1.0$ around the jet axis was compared to the simulation. The one-sigma upper limit on the level of agreement between data and simulation was found to be a percentage shift of

$$
\exp \left(2.466-0.0736 \times E_{T}\right)+1.4379
$$

- This shift was combined in quadrature with an uncertainty of $1 \mathrm{Gel}$ representing 
the amount of energy outside $R=1.0$. Pseudo-experiments then determined the shift to the $W$ mass, the soft gluon uncertainty. These jet energy scale uncertainties are listed in table 4.7 .

\begin{tabular}{|c|c|c|}
\hline Quantity varied & median $M_{W}\left(\mathrm{GeV} / c^{2}\right)$ & Sys. unc. \\
\hline Standard & 79.8 & \\
\hline \multicolumn{3}{|c|}{ Calorimeter Stability, $1 \%$ of raw $E_{T}$} \\
\hline up & 81.0 & $0.7 \mathrm{GeV} / c^{2}$ \\
\hline down & 79.5 & \\
\hline \multicolumn{3}{|c|}{ Calorimeter response, $(\approx 2.5 \% \oplus$ mult. int. $)$} \\
\hline up & 82.0 & $1.7 \mathrm{GeV} / c^{2}$ \\
\hline down & 78.6 & \\
\hline \multicolumn{3}{|c|}{ Soft gluon $\oplus 1 \mathrm{GeV}$} \\
\hline up & 82.7 & $2.4 \mathrm{GeV} / c^{2}$ \\
\hline down & 77.9 & \\
\hline
\end{tabular}

Table 4.7: Systematic uncertainty on the $W$ mass measurement due to uncertainties in the jet energy scale.

\section{Results}

We the $W$ mass to be

$$
78.3 \pm 5.1(\text { stat }) \pm 3.0(\text { syst }) \mathrm{GeV} / \mathrm{c}^{2}
$$

where the systematic uncertainty is summarized in table 4.8 . 


\begin{tabular}{||c|c||}
\hline $\begin{array}{c}\text { Soft gluon effects } \\
\text { Calorimeter effects }\end{array}$ & $2.4 \mathrm{GeV} / c^{2}$ \\
Monte Carlo statistics & $0.5 \mathrm{GeV} / c^{2}$ \\
Fitting constraints & $0.2 \mathrm{GeV} / c^{2}$ \\
Fitted width & $0.2 \mathrm{GeV} / c^{2}$ \\
Background level and shapes & $0.2 \mathrm{GeV} / c^{2}$ \\
Sideband events & $0.1 \mathrm{GeV} / c^{2}$ \\
\hline TOTAL & $3.0 \mathrm{GeV} / c^{2}$ \\
\hline
\end{tabular}

Table 4.8: Summary of the systematic uncertainties on the measurement of the hadronic $W$ mass. 


\subsection{Significance of the Result}

We show two techniques to evaluate the significance of this peak. The first uses a simple mass-window criterion. The second uses the likelihood method, and uses as an estimator the difference in likelihoods with and without a $W$ signal term.

\subsubsection{Mass Window Technique}

We define the $W$ mass window between 60 and $100 \mathrm{GeV} / c^{2}$. We note that eight of our eleven events fall in this range, and another barely misses with a mass of 59.1 $\mathrm{GeV} / c^{2}$

In $W+$ QCD Monte Carlo, $33.7 \%$ of the dijet combinations fall in this mass window. From binomial statistics, we find that the probability of eight, nine, ten, or eleven events in the mass window is $0.95 \%$. This corresponds to a $2.3 \sigma$ effect. For $t \bar{t}$ Monte Carlo, $36.5 \%$ of the dijet combinations which pass the double-tag criteria but do not have both jets matching to quarks from $W$ decay fall in the window. Assuming this to be the source of background, the probability is $1.68 \%$ or a $2.1 \sigma$ effect.

If we include a $W$ signal and run pseudo-experiments with the expected background rate, the probability of getting eight or more events in the mass window rises to $23 \%$.

\subsubsection{Likelihood Technique}

In order to be more sensitive to the shape of the events in the peak, we use a technique which measures the likelihood difference between fits with and without a $W^{*}$ contribu- 
tion. This difference is then compared with the results of pseudo-experiments. The W signal term is set to a Gaussian with fixed mass equal to the generated mass, 80.4 $\mathrm{GeV} / c^{2}$, and a resolution of $11.7 \mathrm{GeV} / \mathrm{c}^{2}$, as measured from Monte Carlo.

When we fit the data with and without this fixed Gaussian term, we see a difference of 4.35 units of log-likelihood. In 20000 pseudo-experiments done with $1.3 \pm 0.4$ background events and the remainder from top with no hadronic $W$ contribution, we see a difference larger than this in 34 experiments. This gives a Gaussian significance of $2.9 \sigma$. If we allow the expected $W$ signal, this probability rises to $15 \%$.

\subsection{0 $t \bar{t}$ Event Reconstruction}

\subsubsection{Introduction}

Once two $b$ quarks are tagged, and the untagged jets are found to have an invariant mass near the $W$ mass, we are left with a sample that is almost purely $t \bar{t}$. and reconstructing the $t \bar{t}$ event becones simplified. Only two twofold ambiguities remain: choosing which $b$ quark goes with which top decay, and which of the two neutrino $z$ momentum solutions to take.

In this section, we present a technique for measuring the top mass which does not use kinematic fitting. We resolve the above ambiguities by choosing the configuration where the three-body masses match most closely. We then take the top mass to be the average of the values obtained from the hadronic and leptonic sides. We show that this technique measures the top mass measurement nearly as well as the fitter 


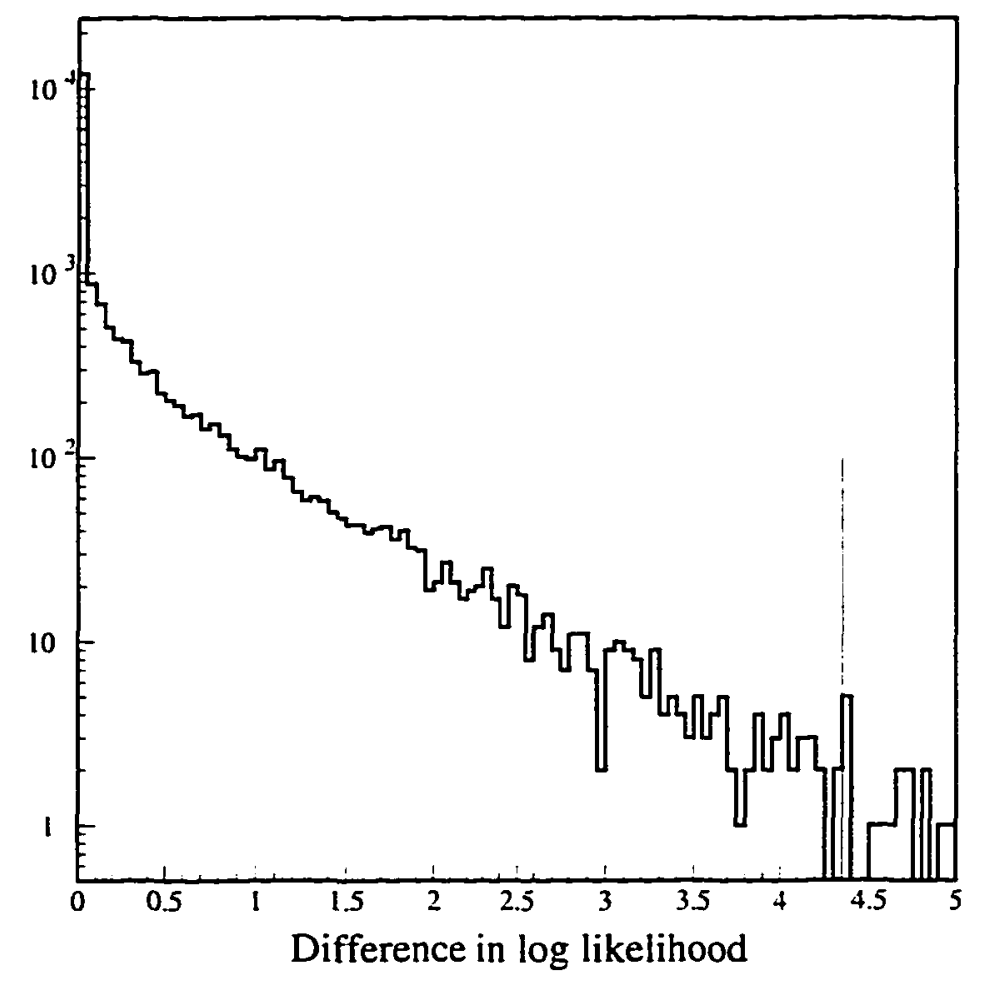

Figure 4.19: The change in likelihood with and without a fixed Gaussian signal term, for simulated experiments which use only background events. The line indicates the value of 4.35 from the data.

technique [39].

\subsubsection{Kinematic fitting of the top mass}

$\mathrm{CDF}$ uses a kinematic fitting technique to extract a top mass. The input values are as follows: fully corrected jets are used, and the jet mass to be is taken to be $0.5 \mathrm{GeV} / c^{2}$ for $W$ jets and $5 \mathrm{GeV} / c^{2}$ for $b$ jets. The $\not_{t}$ is found by first taking the unclustered $E_{T}$ as anything which doesn't balance against the raw $\mathbb{E}_{t}$, lepton, or clustered jets. 
Next the unclustered energy is corrected by a factor of 1.6, the electron and jets are corrected, and the transverse energy components are rebalanced to form the corrected $E_{t}$

Five vertices are considered: the production vertex, two top decay vertices, and two $W$ decay vertices. The masses of the top quarks are required to be equal, and the $W$ mass constraint is applied at both $W$ vertices. The result is a 2-constraint fit. All permutations of jets are assigned to the partons. Also, two solutions exist for the neutrino $z$ momentum. This results is 24 fit solutions. All are tried. The lepton and jet energies are allowed to vary within their experimental uncertainties, and the configuration and top mass which minimize the overall $\chi^{2}$ of the event is chosen. An event is rejected if no permutation has $\chi^{2}<10$.

The distribution of top masses from the events is then fitted to Monte Carlo templates for background samples, and for $t \bar{t}$ samples of various top masses. The negative logarithms of the likelihoods are plotted as a function of the top mass in the templates, and fit to a parabola. The top mass is given by the minimum of this parabola, and its statistical uncertainties are given by the mass values where the function increases by half a unit of log-likelihood.

\subsubsection{Three-body balancing technique}

As in [39], we only consider events with a hadronic $W$ mass in the range $60-100$ $\mathrm{GeV} / c^{2}$. In some events the leptonic $W$ transverse mass exceeds the $W$ mass. In this case we only take the real part of the quadratic solution for the neutrino $p_{z}$. We have 
also looked at scaling down the $Z_{t}$ to make a transverse mass of $M_{W}$, but doing this biases the returned top mass low without significantly improving resolution.

When considering how to combine the information from the leptonic and hadronic side of the event, we found that giving the two masses equal weighting gave the narrowest top mass peak.

Finally, in order to compare results with the mass fitting analyses, we choose a cut on the difference in three-body masses, analogous to the $\chi^{2}$ cut. The fitter $\chi^{2}$ cut rejects only $3 \%$ of the double-tagged events with a $W$ mass in the window. To obtain the same acceptance, we choose a mass difference cut of $70 \mathrm{GeV} / \mathrm{c}^{2}$. The mass difference distribution from Monte Carlo is shown in figure 4.20.

Figure 4.21 shows the mass reconstruction for events from $175 \mathrm{GeV} / c^{2} t \bar{t}$. Monte Carlo. The correct configuration is found $53 \%$ of the time, and $70 \%$ of the events have the four highest $E_{T}$ jets match to the original quarks.

The mass extraction is then done using the usual likelihood method. For the background shape, we again weight by the double-mistag probability.

\subsubsection{Comparison to fitting technique}

Figures 4.22 and 4.23 show how the two mass measurement techniques compare in both mean and variance of the returned mass peaks for templates of each value of top mass. It can be seen that the balancing technique gives widths $1.5-2.5 \mathrm{GeV} / \mathrm{c}^{2}$ wider, and tracks the top mass slightly better.

The distribution of pulls from $175 \mathrm{GeV} / c^{2} t \bar{t}$. Ionte Carlo is shown in figure 4.24 . 


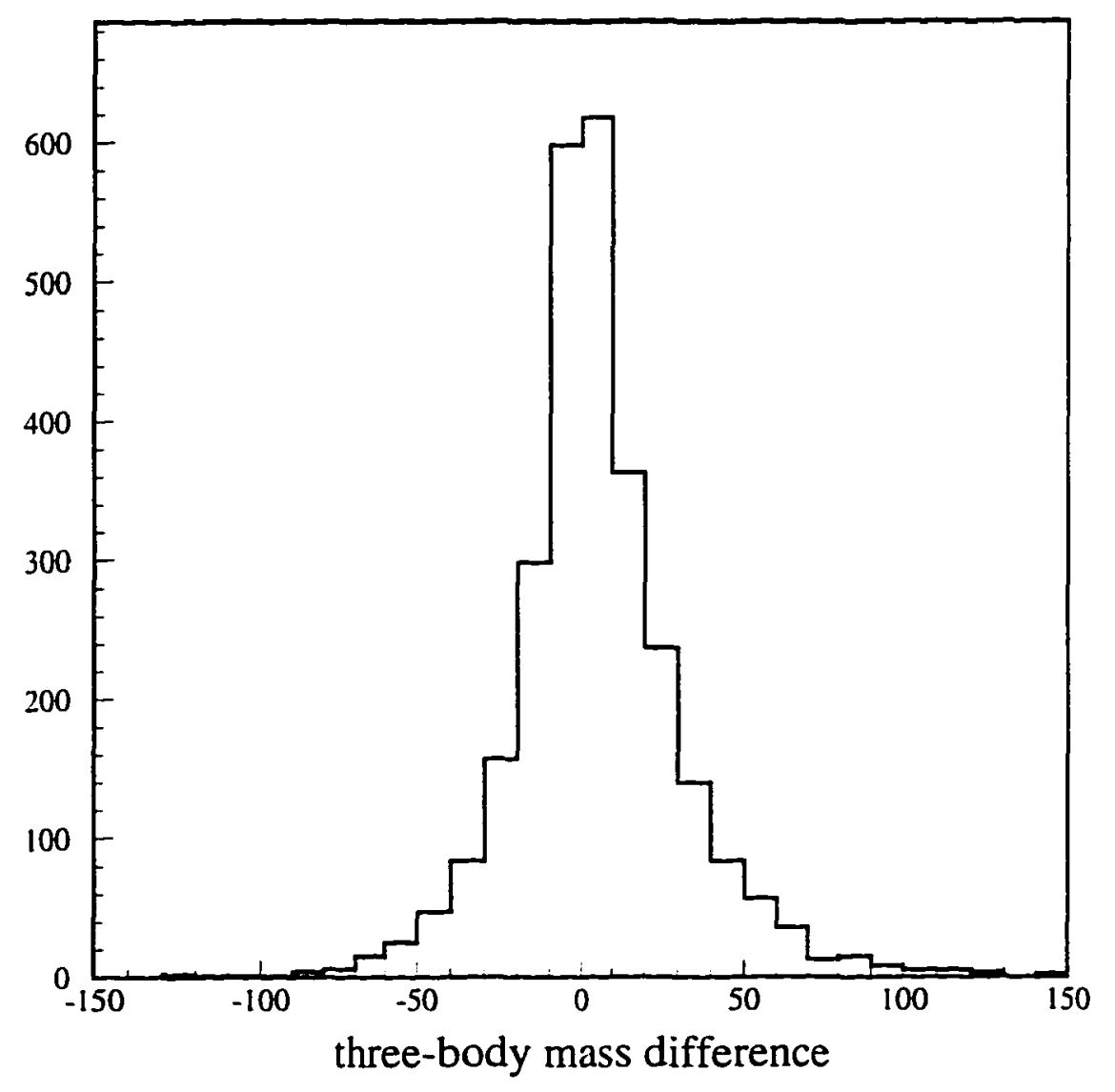

Figure 4.20: The mass difference between the leptonic and hadronic sides of the reconstructed top decay, from $t \bar{t}$ Monte Carlo. 


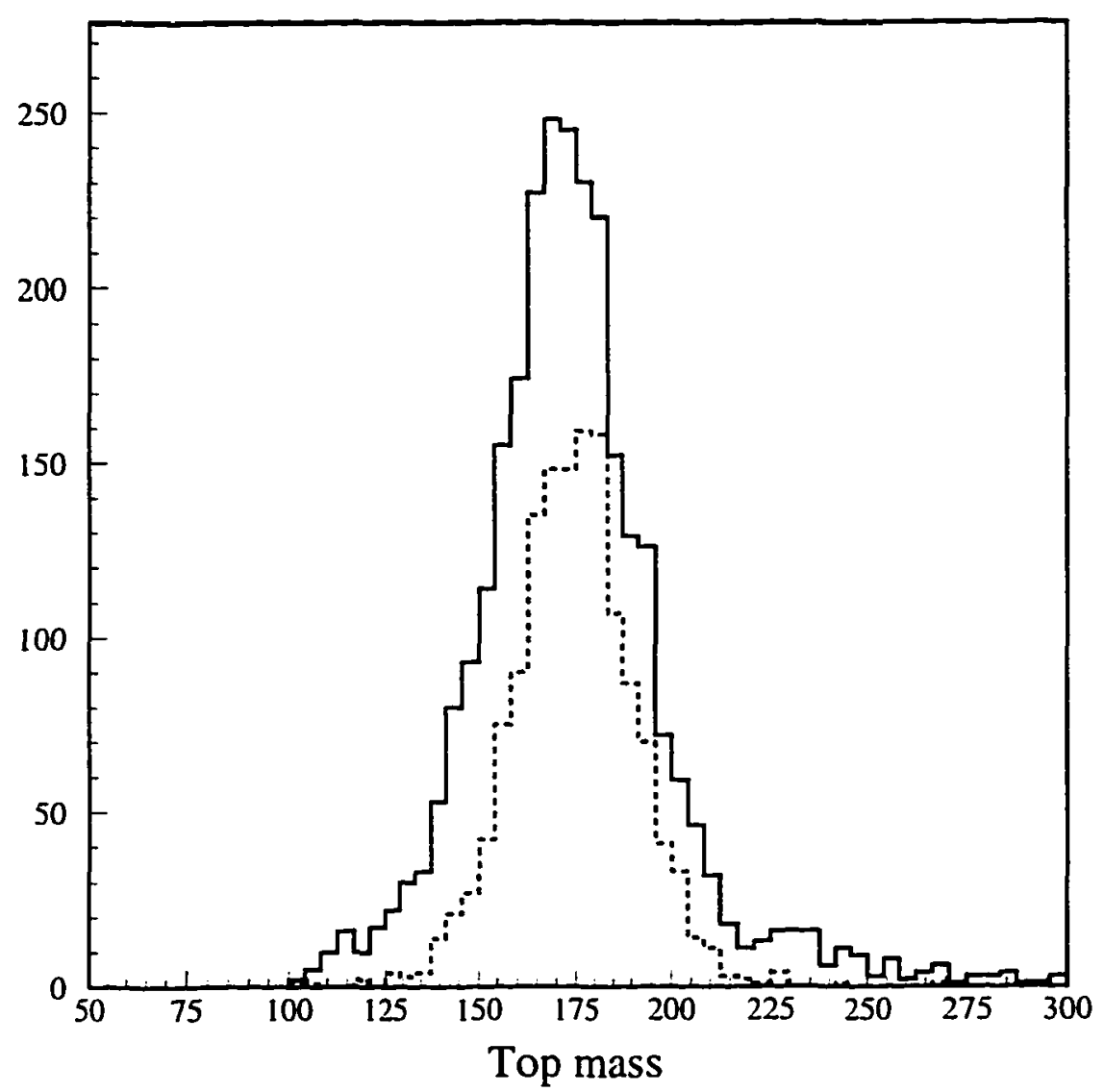

Figure 4.21: The reconstructed top mass for $175 \mathrm{GeV} / \mathrm{c}^{2}$. Wonte Carlo. The dotted line is the shape of events in the correct configuration. 


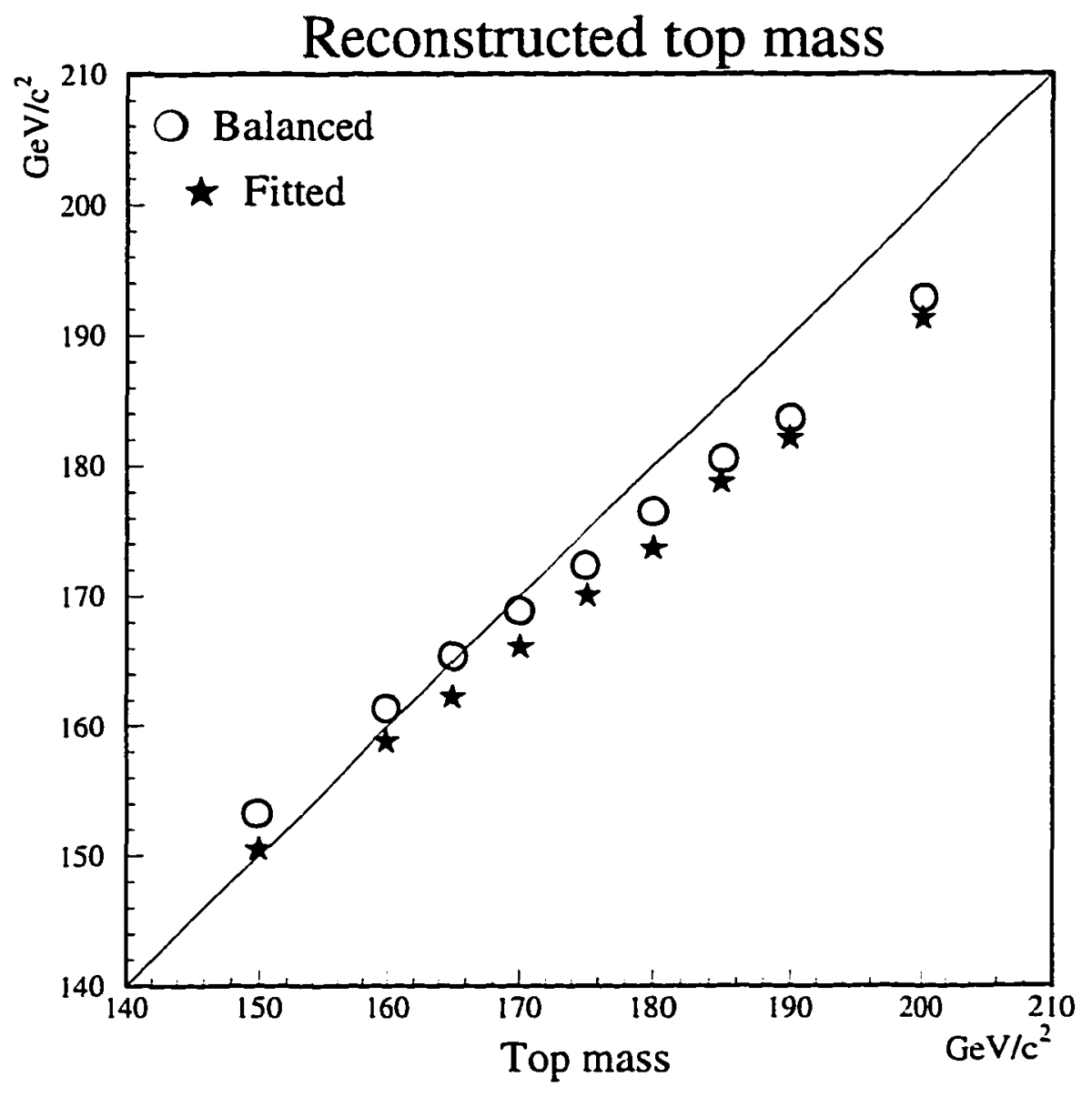

Figure 4.22: Mean reconstructed top mass, obtained by fitting to a Gaussian. for the mass-balancing and fitting techniques. 


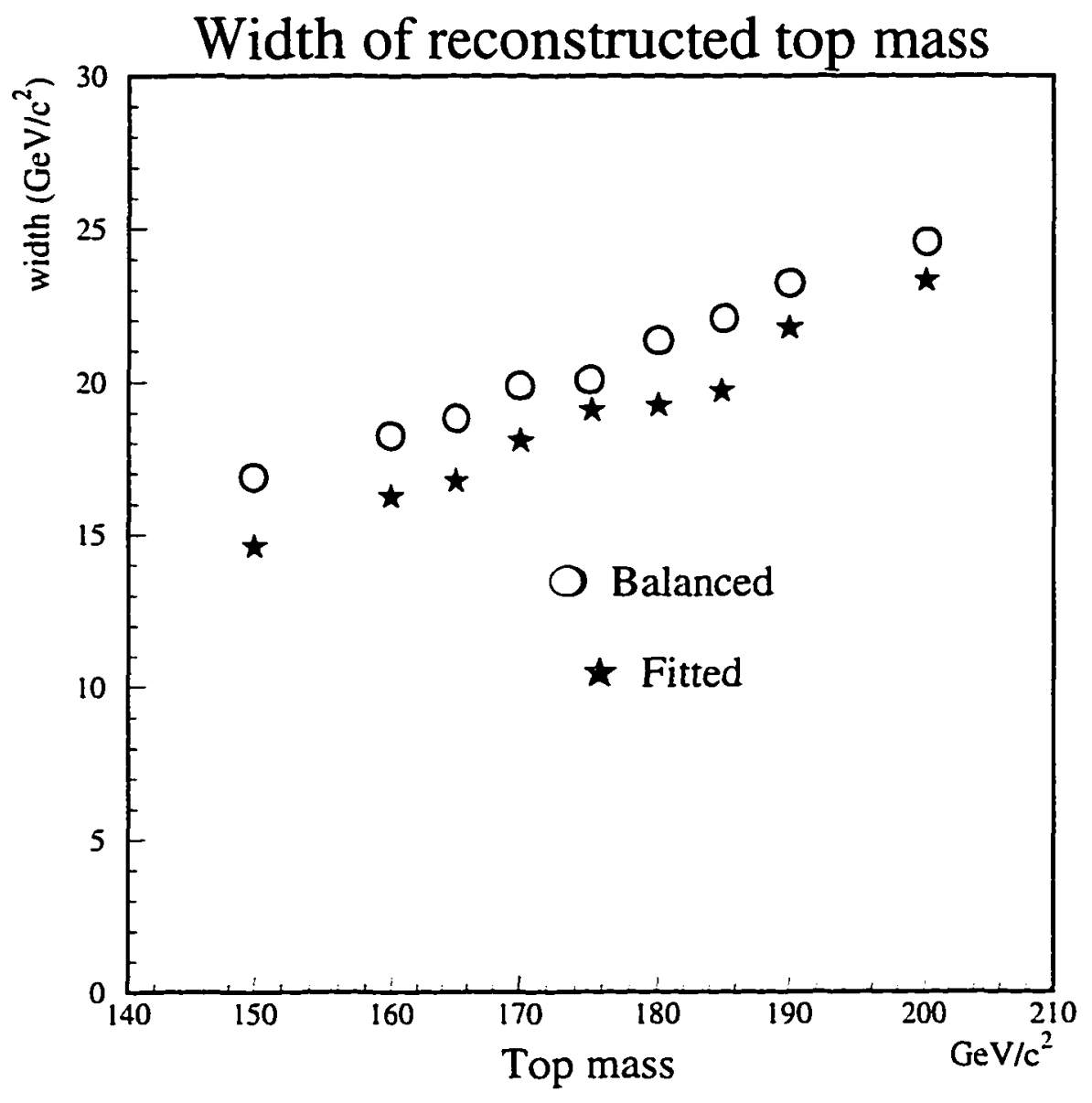

Figure 4.23: The Gaussian widths of the top mass distributions for the two techniques. 
and seen to be good.

\subsubsection{Mass-balancing results from data}

Of the eight events in the $W$ mass window, one fails the mass difference cut. The results are summarized in table 4.9 , and in figure 4.25 .

\begin{tabular}{||c|c|c|c|c|}
\hline Run & Event & $M_{\text {top }}\left(\mathrm{GeV} / c^{2}\right)$ & $M_{3 j}\left(\mathrm{GeV} / c^{2}\right)$ & $M_{\ell \nu j}\left(\mathrm{GeV} / c^{2}\right)$ \\
\hline 40758 & 44414 & 170.9 & 170.7 & 171.1 \\
59698 & 31639 & 192.6 & 180.9 & 204.2 \\
63247 & 65096 & 165.1 & 159.5 & 172.0 \\
64721 & 229200 & 195.4 & 174.1 & 216.7 \\
65581 & 322592 & fails $\Delta M$ cut & 235.6 & 163.2 \\
67824 & 281883 & 178.2 & 159.4 & 197.0 \\
67971 & 55023 & 193.9 & 202.3 & 185.4 \\
68464 & 547303 & 155.5 & 158.2 & 152.8 \\
\hline
\end{tabular}

Table 4.9: Results of the top mass measurement using the three-body mass balancing technique.

Doing a likelihood fit to the top and background templates yields a top mass of $182.3 \pm 9.6 \mathrm{GeV} / c^{2}$ 


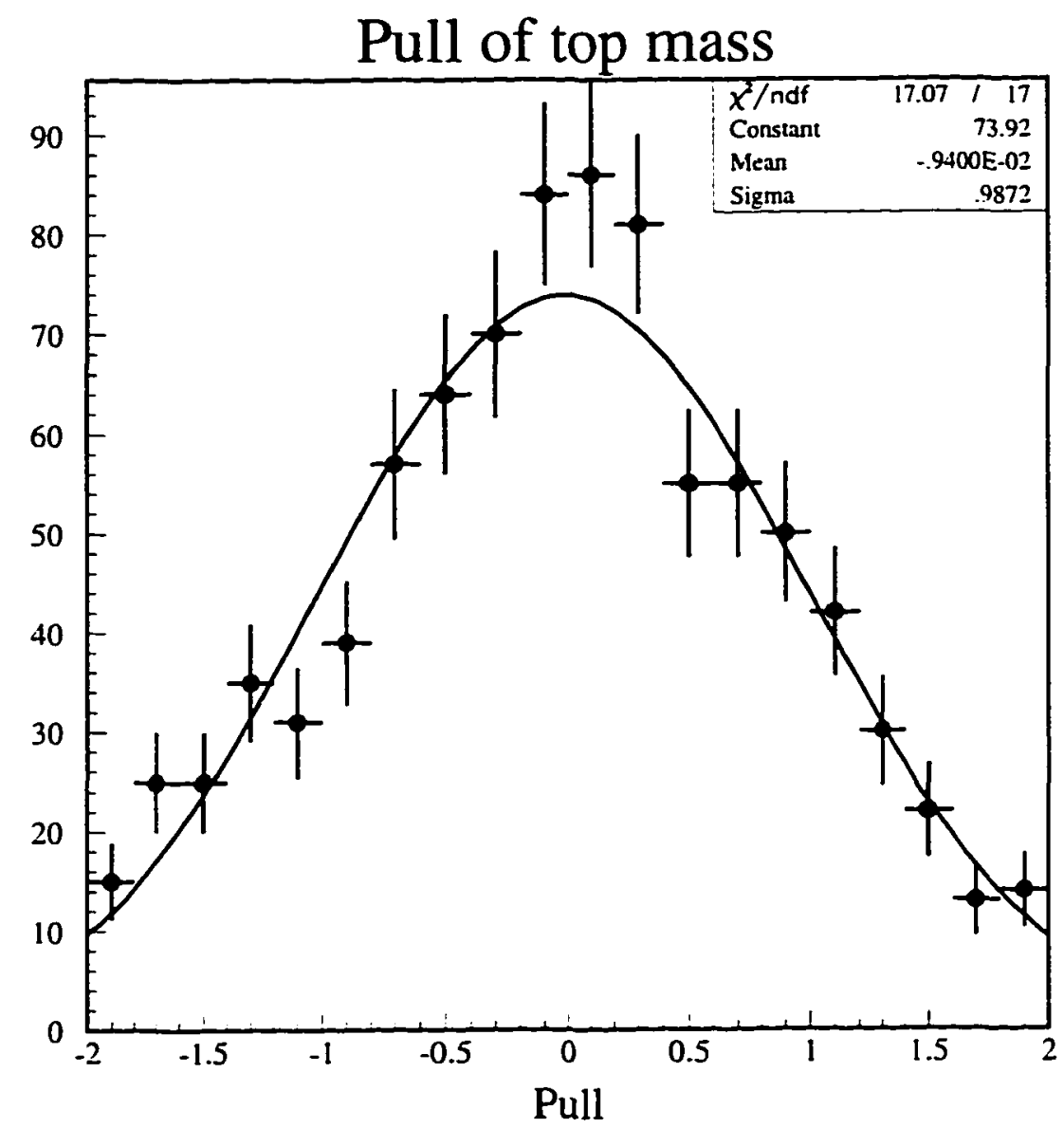

Figure 4.24: The distribution of pulls of the top mass, from pseudo-experiments. 


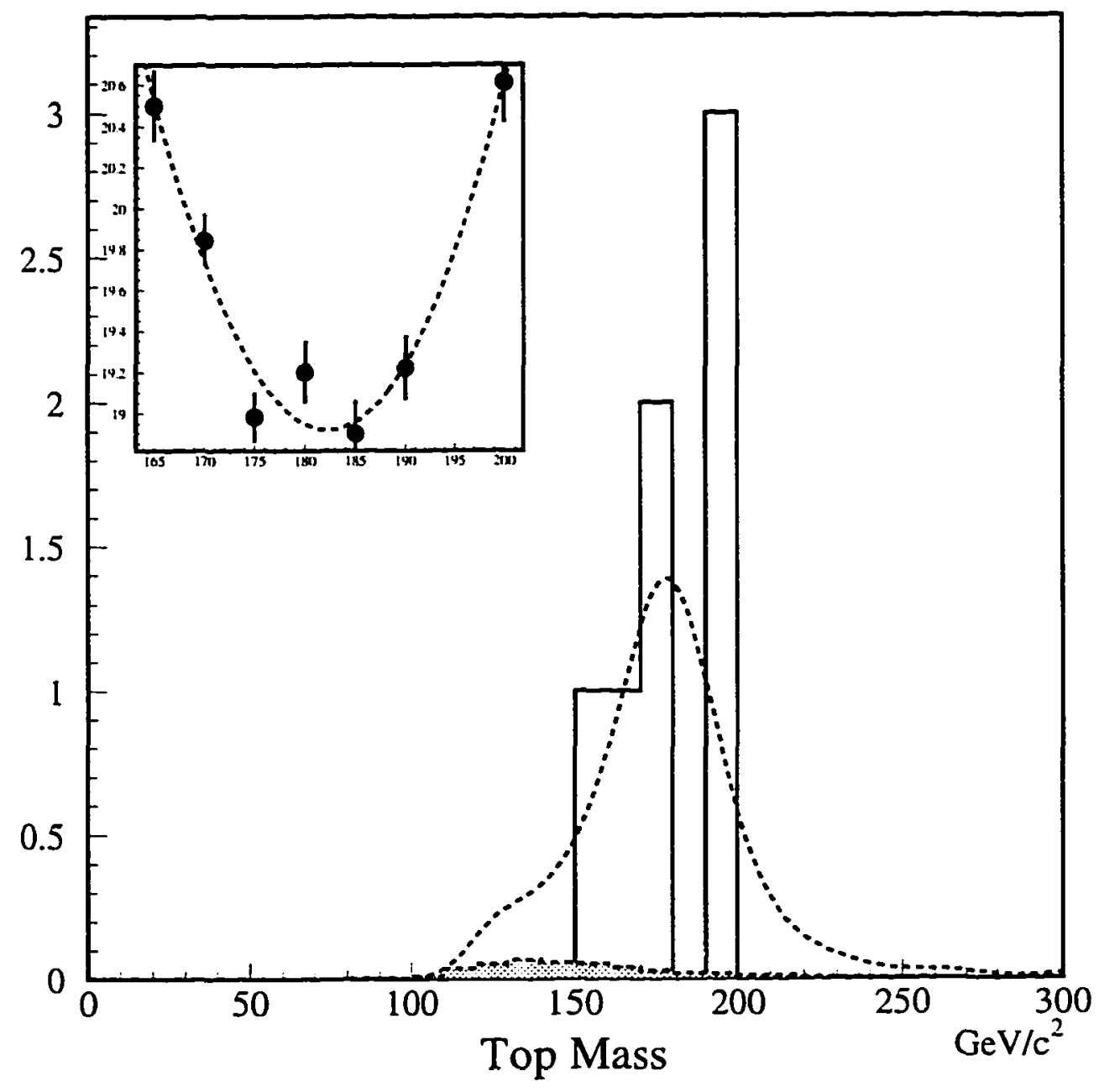

Figure 4.25: The top masses obtained from these seven events. A likelihood fit to Monte Carlo templates of different top masses yields a measured top mass of 182.3 $\pm 9.6 \mathrm{GeV} / c^{2}$. 


\subsubsection{Systematic uncertainties}

Wost of the evaluation of systematic uncertainties was done using the same methods as in section 4.8.4. The techniques that differ are described below.

\section{Hard gluon radiation}

In this dataset, $30 \%$ of the events have a gluon jet among the four leading jets. Since the lowest possible gluon fraction is $0 \%$, we take that to be the $2 \sigma$ point, and consider the effects of varying the gluon fraction in pseudo-experiments by $\pm 15 \%$. The resulting shift is $1.2 \mathrm{GeV} / \mathrm{c}^{2}$.

Another way to evaluate this uncertainty is to eliminate initial-state gluon radiation from the Monte Carlo input to the pseudo-experiments. Doing this shifts the median returned top mass down by $3.1 \mathrm{GeV} / c^{2}$, so we may take half of this as the systematic uncertainty. Final-state radiation effects are expected to be accounted for in the jet energy scale uncertainty.

We take as our final uncertainty the average of these two results, or $1.4 \mathrm{GeV} / \mathrm{c}^{2}$.

\section{Likelihood method}

Some ways to vary the fit technique include altering the constraints on the background, letting the total number of events $N$ float, or changing which points are used in fitting the likelihoods to a parabola. The results are shown in table 4.11. The uncertainty is $0.3 \mathrm{GeV} / c^{2}$. 


\begin{tabular}{|c|c|c|c||}
\hline Gluon fraction & Result & Mean Uncertainty & Sys. unc. \\
\hline $0 \%$ & 181.9 & 12.4 & 2.0 \\
$15 \%$ & 181.0 & 13.8 & 1.1 \\
$20 \%$ & 180.9 & 13.8 & 1.0 \\
$30 \%$ (HERWIG) & 179.9 & 13.9 & \\
$40 \%$ & 179.4 & 15.1 & 0.5 \\
$45 \%$ & 178.7 & 15.1 & 1.2 \\
$65 \%$ & 178.9 & 17.8 & 1.0 \\
\hline
\end{tabular}

Table 4.10: Systematic uncertainty on the top mass due to uncertainty on the amount of hard gluon radiation.

\section{Event configuration}

For this, we look to see if different event configurations will also give reasonable solutions for the top event. To define "reasonable", we note that the width of the plot of mass imbalance between the two sides of the event has a width of $21 \mathrm{GeV} / c^{2}$, so we look for configurations with a mass imbalance less than $21 \mathrm{GeV} / c^{2}$ worse than the best balance. In only one event, Run 67971 Event 55023 , we see that a different neutrino solution lowers the returned mass from $193.9 \mathrm{GeV} / c^{2}$ to $188.0 \mathrm{GeV} / c^{2}$, and the imbalance becomes $11.6 \mathrm{GeV} / c^{2}$ worse. So we use the second-best top mass for this event as input to the likelihood fit, and the fit returns a top mass of (181.3 \pm 8.9) $\mathrm{GeV} / c^{2}$, leading to an uncertainty of $1.0 \mathrm{GeV} / c^{2}$. 


\begin{tabular}{||c|c||}
\hline Fit technique & Result $\left(\mathrm{GeV} / c^{2}\right)$ \\
\hline Default & $182.3 \pm 9.6$ \\
free bg & $182.3 \pm 9.6$ \\
fixed bg & $182.6 \pm 9.9$ \\
free $N$ & $182.5 \pm 9.1$ \\
fit range $170-200$ & $182.4 \pm 9.5$ \\
fit range $165-190$ & $182.3 \pm 9.6$ \\
fit range $160-200$ & $182.5 \pm 9.1$ \\
\hline
\end{tabular}

Table 4.11: Systematic uncertainty on the top mass due to fit technique.

\subsubsection{Results}

Using a simple three-body balancing technique, we measure the top mass in doubletagged events to be $182.3 \pm 9.6$ (stat) \pm 4.9 (syst) $\mathrm{GeV} / c^{2}$.

\subsection{Measurement of the Jet Energy Scale}

\subsubsection{Introduction}

The position of the $W$ mass peak provides information about how accurately the energies measured in our calorimeter correspond to the energies of the original quarks. This jet energy scale is a large systematic uncertainty in many measurements at CDF. including the top mass measurement. 


\begin{tabular}{||c|c||}
\hline Quantity varied & Sys. uncert. $\left(\mathrm{GeV} / \mathrm{c}^{2}\right)$ \\
\hline Jet energy scale \\
\hline Detector effects & 3.2 \\
Soft gluon effects & 2.7 \\
\hline Monte Carlo statistics & 1.8 \\
Hard gluon radiation & 1.4 \\
Event configuration & 1.0 \\
Different MC generator & 0.7 \\
Likelihood method & 0.3 \\
Background shape & 0.0 \\
Background level & 0.0 \\
\hline
\end{tabular}

Table 4.12: Systematic uncertainty on the top mass result from the three-body balancing technique. 


\subsubsection{Preliminary measurement}

Here we attempt to translate our $W$ mass measurement of $78.3 \pm 5.9 \mathrm{GeV} / c^{2}$ into a measurement of the jet energy scale. To do this, we run ensembles of pseudoexperiments using input data which was shifted in raw $E_{T}$ by $\pm 2 \%, \pm 5 \%$, and $\pm 10 \%$. We find the median $W$ mass returned for each shifted ensemble, and make a linear function to translate between the returned $W$ mass and the jet energy scale. From figure 4.26, we see that our $W$ mass measurement corresponds roughly to a measurement of the jet energy scale of $(-2.5 \pm 8.8) \%$.

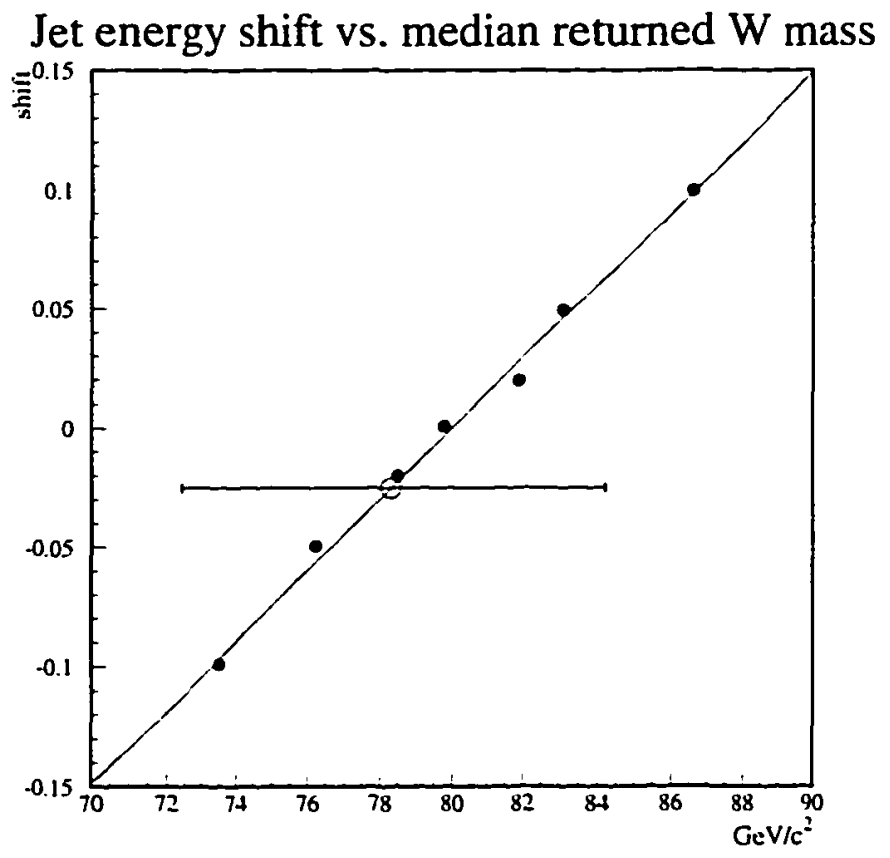

Figure 4.26: The relation between jet energy scale and median returned $W$ mass from ensembles of pseudo-experiments. The large point represents the measurement in the data, which translates into a rough estimate of the jet energy scale of $(-2.5 \pm 8.8) \%$. 


\subsubsection{Likelihood method}

Another way to do this measurement is to perform a likelihood fit to templates made from double-tagged events with various jet energy scales. We use HERWIG Monte Carlo for the templates, and constrain the background with a Poisson term centered at 1.3 events. The background shapes are weighted by their double-mistag probabilities. Doing this yields the likelihood distribution shown in figure 4.27 , from which we obtain a jet energy scale measurement of $(-2.4 \pm 8.0) \%$.

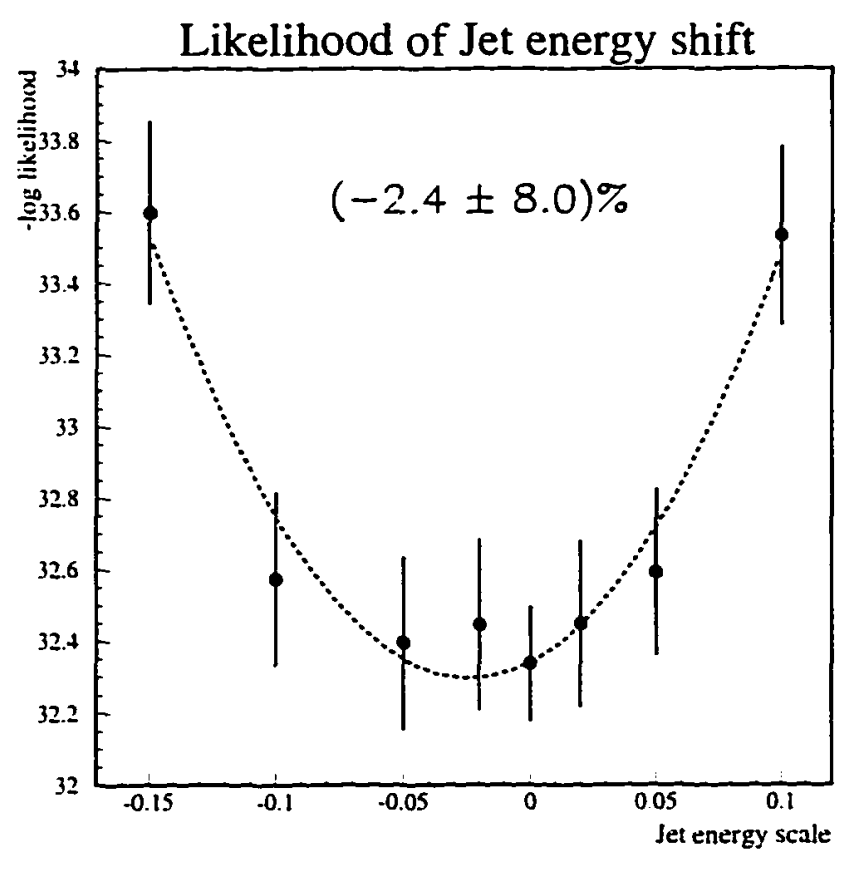

Figure 4.27: The results of a likelihood fit to templates with various jet energy scale shifts. 


\subsubsection{Systematic uncertainties}

The systematic uncertainties are evaluated in much the same way as the previous measurements in this work. We must also include the uncertainty on the $W$ mass. which the latest Particle Data book lists as $80.33 \pm 0.15 \mathrm{GeV}$. This translates into a $0.2 \%$ uncertainty. The systematic uncertainties are summarized in table 4.13 .

\begin{tabular}{||c|c||}
\hline Quantity varied & Sys. uncert. (\%) \\
Throw out sideband events & 1.8 \\
Hard gluon radiation & 1.4 \\
Top mass in templates & 1.0 \\
Monte Carlo staitistics & 0.7 \\
Background shape & 0.4 \\
True $W$ mass & 0.2 \\
Background constraint & 0.1 \\
\hline
\end{tabular}

Table 4.13: Systematic uncertainties on the measurement of the jet energy scale.

\subsubsection{Results}

We use the hadronic $W$ peak in the double-tagged data to obtain a measurement of the jet energy scale at CDF of

$$
(-2.4 \pm 8.0(\text { stat }) \pm 2.6(\text { syst })) \%
$$




\subsubsection{Future precision}

Table 4.14 summarizes the mean statistical precision of the jet energy scale measurement pseudo-experiments, as a function of the number of events in the sample. This assumes the current mix of signal and background (1.3 background for every 11 signal events).

\begin{tabular}{||c|c||}
\hline Number of events & Statistical precision (\%) \\
\hline 11 & 9.2 \\
50 & 5.1 \\
100 & 3.6 \\
$300\left(1 \mathrm{fb}^{-1}\right)$ & 2.1 \\
\hline
\end{tabular}

Table 4.14: Expected future precision of the jet energy scale measurement.

\subsection{Determining the Hard Gluon Radiation Content}

Another large systematic uncertainty in the top mass measurement is the amount of hard gluon radiation modelled in the Monte Carlo. As was seen earlier, the purity of the double-tagged $W$ dijet peak depends strongly upon the hard gluon content of $t \bar{t}$ events, because the main background comes from $t \bar{t}$ events with two $b$ jets and one or more jets from hard gluon radiation among the four hardest jets. Because of 
this, it seems possible to use the relative amounts of signal and sideband from this analysis to measure the hard gluon content of $t \bar{t}$ events. We define the gluon fraction to be the fraction of $t \bar{t}$ events where the four highest $E_{T}$ jets do not correspond to the four generated partons. Until recently, this definition was the basis of the top mass systematic, and is still the most useful for describing the hadronic $W$ peak.

A cautionary note is in order: this measurement is inherently biased, because we probably wouldn't be doing it if the $W \rightarrow j j$ peak weren't so clean in the first place. This section is primarilary meant to show that a measurement of the hard gluon radiation in $t \bar{t}$ events is possible in the double $b$-tagged sample.

\subsubsection{Preliminary look}

We begin by using the fraction of events in the mass window between 60 and $100 \mathrm{Gel}$ as an estimator. We use a toy Monte Carlo to study this behavior. We first include a Poisson-distributed non-t $t \bar{t}$ background with a mean of 1.3 events for every eleven events total. Next, we randomly split the remaining events between hadronic $W$ s and top combinatorics, with $f_{W}$ determined from $f_{g}$. We then assign each background event a $33 \%$ chance of falling in the mass window, each top combinatoric event a $36 \%$ chance, and each hadronic $W$ an $87 \%$ chance. We then run 10000 experiments of eleven events each for various values of $f_{g}$, and determine which fraction of the experiments result in eight or more events in the window. The results are summarized in table 4.15. The distributions for the number of events in the window for gluon fractions of $25 \%, 55 \%$, and $85 \%$ are shown in figure 4.28 . 


\begin{tabular}{||c|c|c||}
\hline$f_{g}$ & $\%$ with $\geq 8$ in window & \% with 8 in window \\
\hline 0 & $64.4 \%$ & $27.0 \%$ \\
$5 \%$ & $60.9 \%$ & $27.0 \%$ \\
$10 \%$ & $56.9 \%$ & $26.1 \%$ \\
$25 \%$ & $44.9 \%$ & $23.3 \%$ \\
$35 \%$ & $37.1 \%$ & $20.7 \%$ \\
$45 \%$ & $26.9 \%$ & $18.3 \%$ \\
$55 \%$ & $22.9 \%$ & $15.1 \%$ \\
$65 \%$ & $16.9 \%$ & $12.0 \%$ \\
$75 \%$ & $11.4 \%$ & $8.3 \%$ \\
$85 \%$ & $7.2 \%$ & $5.4 \%$ \\
$99 \%$ & $3.5 \%$ & $2.6 \%$ \\
\hline
\end{tabular}

Table 4.15: The fraction of pseudo-experiments with eight (or more) events in the mass window $60-100 \mathrm{GeV} / c^{2}$, as a function of the fraction of input events with one or more gluon jets. 

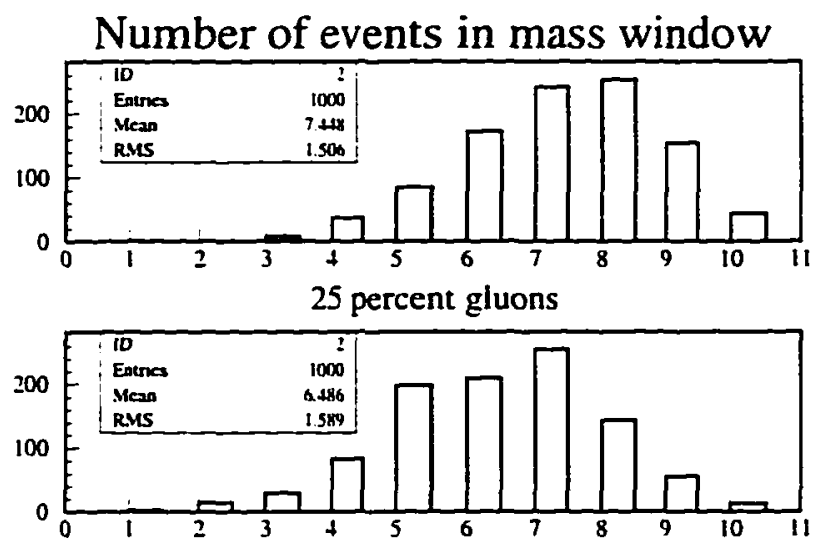

55 percent gluons

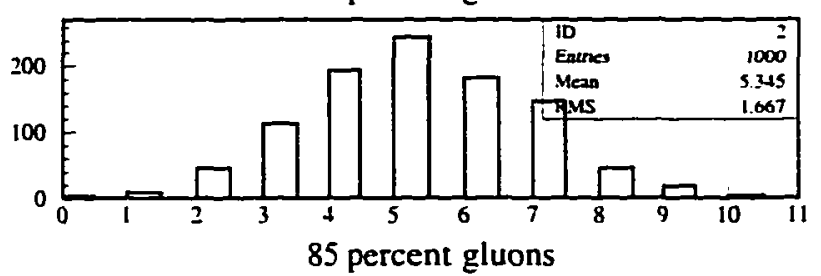

Figure 4.28: The number of events in a mass window of $60-100 \mathrm{GeV} / \mathrm{c}^{2}$ for 10000 pseudo-experiments with gluon fractions of $25 \%, 55 \%$, and $85 \%$.

From the right column of table 4.15 , we see that the probability of getting eight events peaks for a gluon fraction of zero, indicating that mistags and background are sufficient to account for the observed sidebands. This probability drops by a factor of $e^{-1 / 2}$ around a gluon fraction of $40 \%$, indicating a one-sigma limit. From the left column of table 4.15 , we see that a one-sided $90 \%$ confidence level limit could be set around a gluon fraction of $80 \%$, and a $95 \%$ limit around $90 \%$. 


\subsubsection{Likelihood fit}

To do this more rigorously, we next fit our data sample against templates with various mixes of gluon and non-gluon $t \bar{t}$ events. The templates are shown in figure 4.29 .
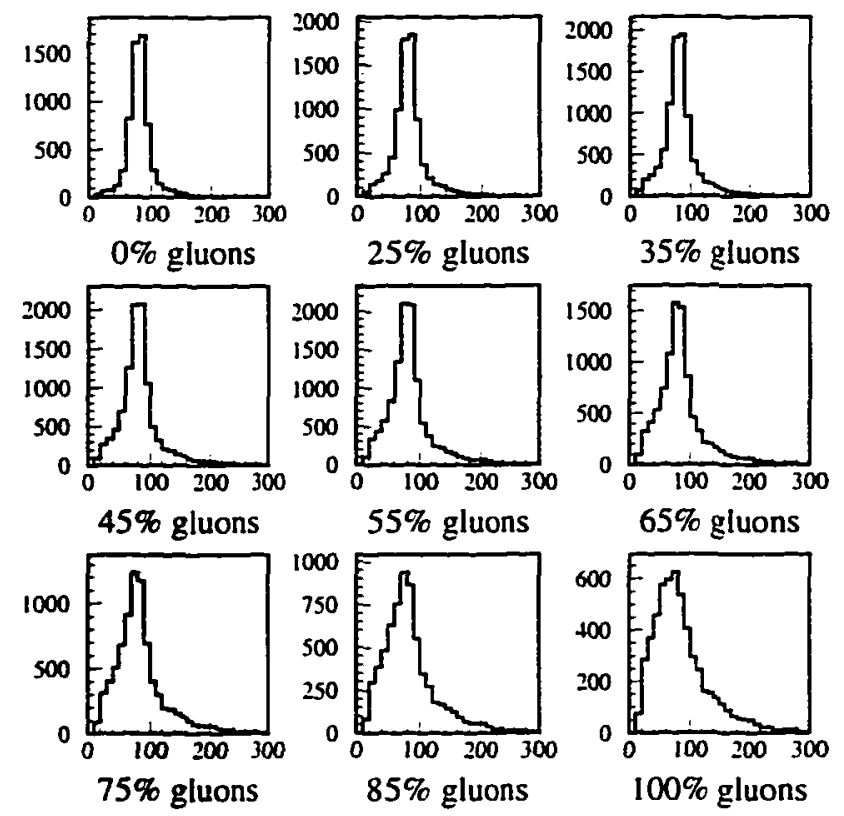

Figure 4.29: Templates with various mixes of gluon and non-gluon $t \bar{t}$ events.

A few decisions remain. Since the background level is important to this result. we must be careful in choosing how to constrain it. Options include using a Poissonshaped constraint with mean 1.3 events, a Gaussian constraint with a width of 0.4 events, corresponding to the uncertainty on the mean background calculation, or a looser Gaussian constraint of width $\sqrt{1.3}$, to allow more statistical fluctuation of background. Also, we may easily fit in terms of either the gluon fraction in double- 
tagged events, or use the Monte Carlo tagging rates for gluon and non-gluon erents to convert this to a pretagged gluon fraction.

The only objective way to rate these methods is by seeing how well pseudoexperiments can return the input gluon fraction. This is shown in figure 4.30 and from this we note two things. First, using pretagged gluon fractions distorts the results significantly. Second, the Poisson background constraint tends to bias the the results high, because the maximum of a Poisson with mean 1.3 falls at about 0.9 . We choose to use a Gaussian background constraint of width $\sqrt{1.3}$.
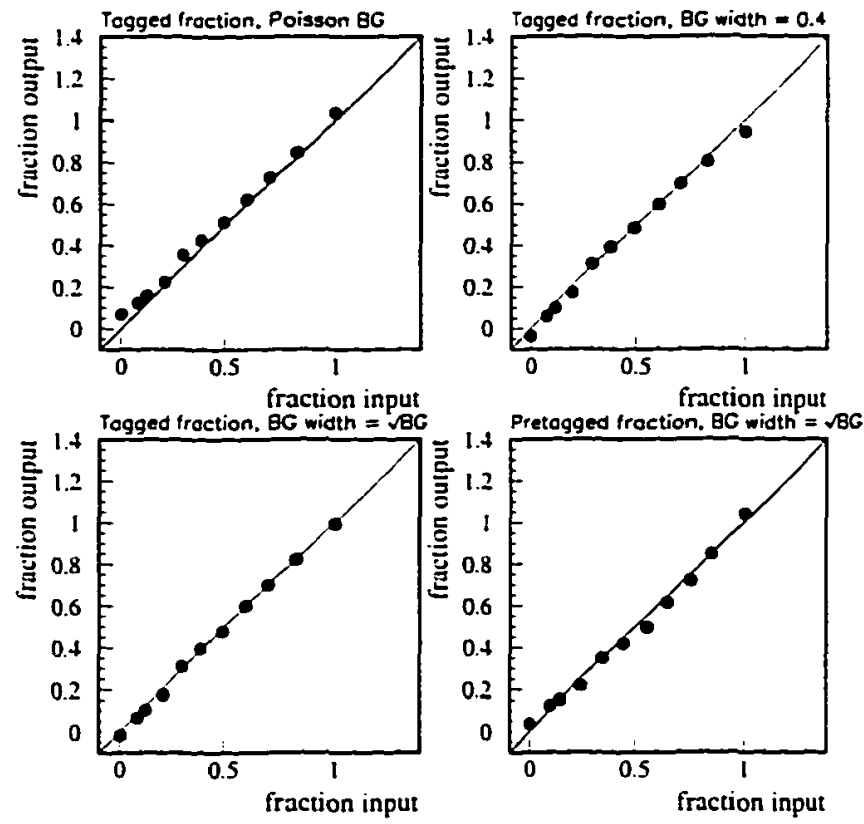

Figure 4.30: Median returned gluon fraction from pseudo-experiments using different fit techniques.

The likelihood fit to the data results in a measured hard gluon fraction of $(-2 \pm$ 102 
$39) \%$, shown in figure 4.31. We will take this to mean that noise events from gluon radiation are not required.

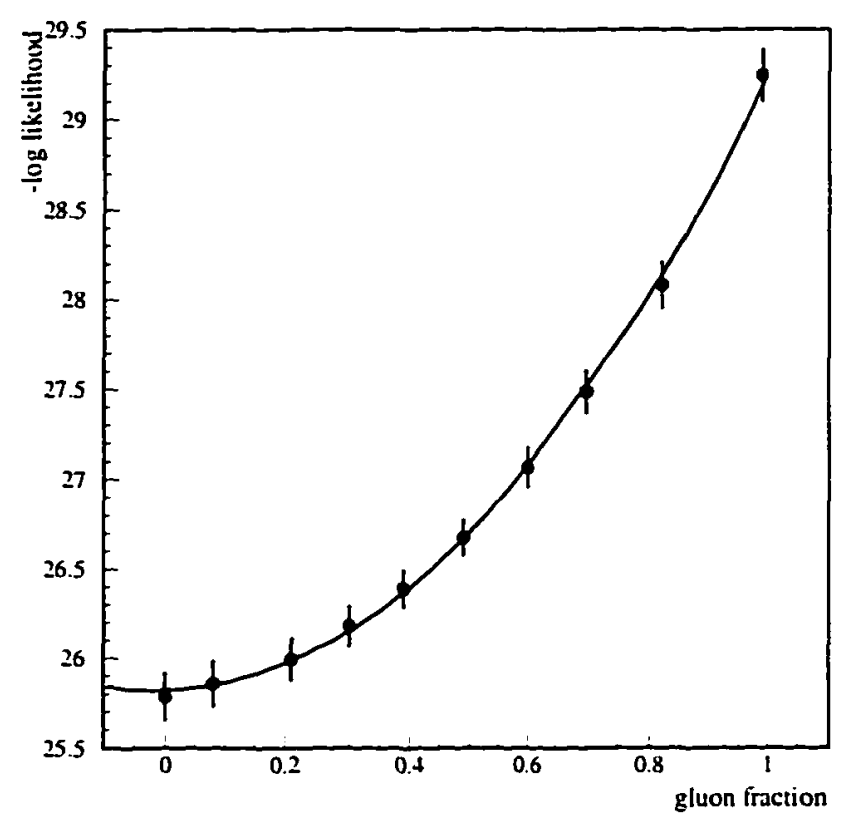

Figure 4.31: Results of the likelihood fit to the data.

\subsubsection{Systematic uncertainties}

The systematic uncertainties on this measurement are listed in table 4.16.

\subsubsection{Results}

We finally get a measurement of the gluon fraction in double-tagged $t \bar{t}$ events of $(-2 \pm 39($ stat $) \pm 14($ syst $)) \%$. Combining the statistical and systematic errors gives a one-sigma limit on the double-tagged gluon fraction $39 \%$, which corresponds to a 


\begin{tabular}{|c|c|}
\hline Quantity varied & Sys. uncert. (\%) \\
\hline Fit to pretagged fraction & 9 \\
\hline Background constraint & 6 \\
\hline Background level & う \\
\hline Throw out sideband events & 4 \\
\hline Monte Carlo statistics & 4 \\
\hline Background shape & 3 \\
\hline Top mass in templates & 2 \\
\hline Jet energy scale & 2 \\
\hline TOTAL & $14 \%$ \\
\hline
\end{tabular}

Table 4.16: Systematic uncertainties to the measurement of the gluon fraction in $t \bar{t}$ events. 
limit on the pretagged gluon fraction of $45 \%$.

To find the $90 \%$ confidence limit, we first shift the mean by one systematic standard deviation, and then follow the likelihood parabola up until the likelihood decreases by a factor of 10 . This yields a limit of $82 \%$, or $85 \%$ pretagged. Similarly, we find a $95 \%$ confidence limit at a gluon fraction of $96 \%$, equivalent to $97 \%$ pretagged.

\subsubsection{Future precision}

Table 4.17 lists the expected future precision of this measurement as a function of the number of events, with 1.3 background events for each eleven events from HERWIG Monte Carlo.

\begin{tabular}{||c|c||}
\hline \# events & Stat. precision \\
\hline 50 & $18 \%$ \\
100 & $12 \%$ \\
$300\left(1 \mathrm{fb}^{-1}\right)$ & $6.4 \%$ \\
\hline
\end{tabular}

Table 4.17: Expected statistical precision of future measurements of the gluon fraction. 


\section{Chapter 5}

\section{Single $W$ production}

In this chapter, we search for the process $W / Z \rightarrow j j$ in a dijet sample. We hope to find a larger signal, although the backgrounds from QCD $2 \rightarrow 2$ processes are huge.

\subsection{UA2 Data}

The UA2 experiment has successfully reconstructed the dijet decays of $W$ and $Z^{0}$ bosons in $4.7 \pm 0.2 \mathrm{pb}^{-1}$ of data [41].

Figure 5.1 shows the UA2 dijet mass spectrum in the $W$ and $Z^{0}$ mass region. The left figure shows the data with entries weighted by $\left(\mathrm{M}_{j j} / 100\right)^{6}$ with three different background fits. The right figure shows the data after background subtraction and an excess which is fitted to a combination of $W$ and $Z^{0}$ signal events. After the background subtraction, they isolated a signal consisting of $5618 \pm 1334$ events $(4.2 \sigma$ significance) which corresponds to a signal to background ratio of $1: 38$ in the 70 to $100 \mathrm{GeV} / c^{2}$ dijet mass region. The measured $W$ mass from fitting the excess (including the $Z^{0}$ contribution) was $79.2 \pm 1.7 \pm 3.4 \mathrm{GeV} / c^{2}$. 

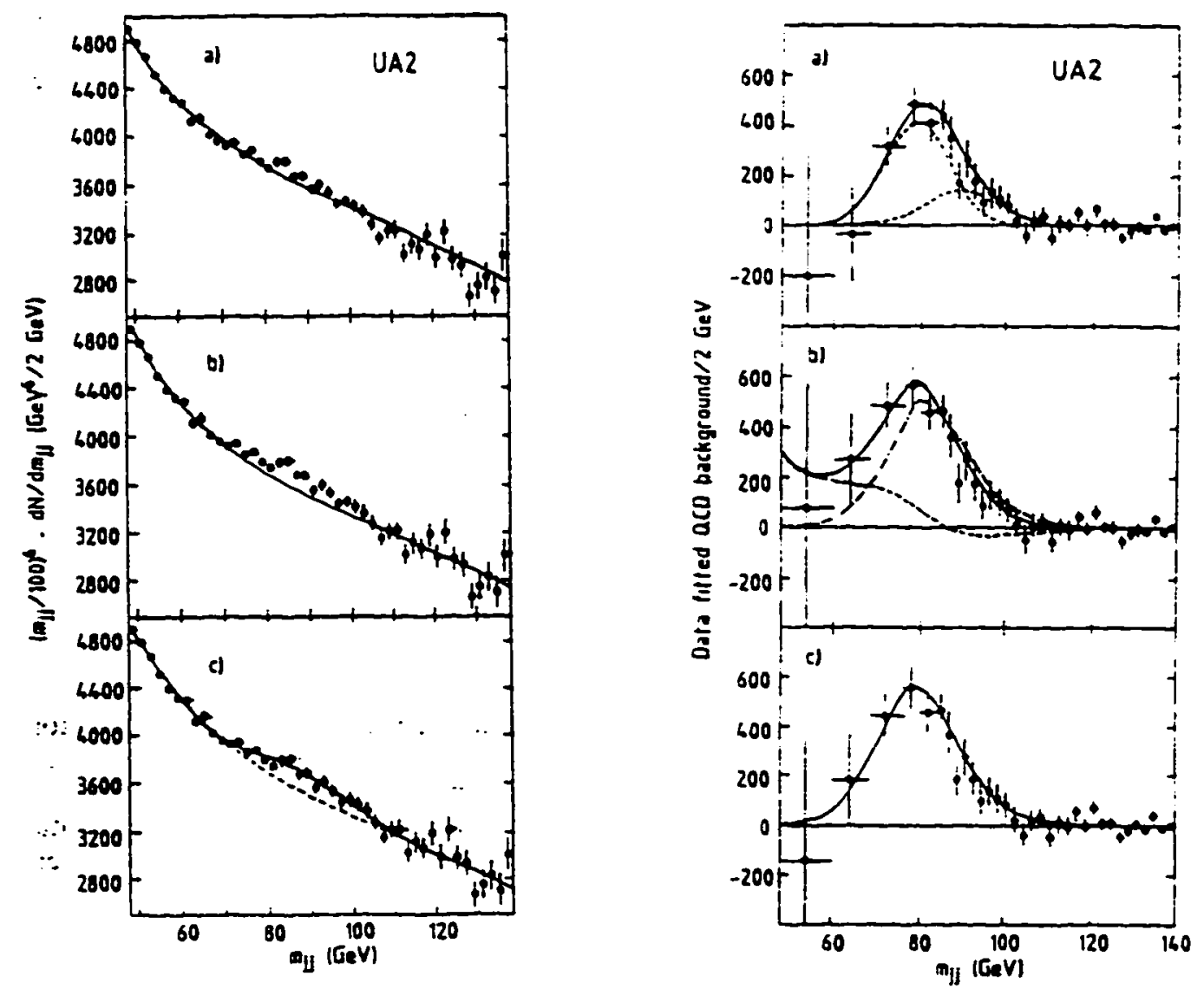

Figure 5.1: UA2 results 


\subsection{Triggers}

In early 1996, a wire in the CTC broke, leaving half of the detector with no tracking. New triggers for physics which required no tracking were implemented to use the $\approx 3 \mathrm{pb}^{-1}$ of luminosity to be given until the Tevatron run ended.

Low $E_{T}$ dijet triggers comprised much of this data, approximately 3.3 million events. Two thresholds were set: a main sample requiring two central trigger clusters with $E_{T} \geq 12 \mathrm{GeV}$ (referred to hereafter as DIJET_12), and a control sample requiring central trigger clusters with $E_{T} \geq 10 \mathrm{GeV}$ (referred to as DIJET_10). The DIJET_10 trigger is prescaled by a factor of $\approx 9$ with the respect to the DIJET_12.

\subsection{Selection Criteria}

In order to find a significant signal, we must find cuts which not only optimize $S / \sqrt{\mathrm{V}}$, but simultaneously give as narrow a $W$ peak as possible. We know that $W$ jets are more central than QCD jets, and that the gluon jets which dominate the background are more likely to radiate more gluons, and thus, more jets. With these facts in mind, we search for cuts which optimize the quantity $\frac{S}{\sigma \sqrt{N}}$, where $\sigma$ is the Gaussian width of the W decays. We use Monte Carlo samples generated using PYTHIA [40] along with detector simulation. We enable all $W$ decay modes, and only count those decays where both jets match within $R<0.4$ of the original quark direction. The background samples include all QCD $2 \rightarrow 2$ processes. For both signal and background, we only include events with dijet masses between 60 and $110 \mathrm{GeV} / c^{2}$. 
We obtain the following selection criteria:

- Jet clustering cone size of 0.7

- $0.1<|\eta|<0.9$, to avoid detector cracks

- Third jet $E_{T}<12 \mathrm{GeV}$

- $\Delta \phi_{j j}>160^{\circ}$ (back-to-back)

- jet electromagnetic energy fraction $<0.95$ (reject $\gamma$ 's and $e^{\prime}$ s).

We now mention other cuts we tried, but did not include in the final analysis.

We may expect improved resolution from more electromagnetic jets, because of the superior resolution of the electromagnetic calorimeter. However, no increase is seen in either Monte Carlo or data resolution of $Z_{t}$. This seems to be because highly electromagnetic jets are often due to hadronic interactions in the electromagnetic calorimeter [42].

Since we expect the signal jets to be more central than QCD, we try cutting on $\cos \theta^{*}$, the cosine of the angle between the lead jet and the beam in the dijet centerof-mass frame. However, since the $\eta$ range is already restricted, this cut is only of marginal benefit. We leave it out for simplicity.

A cut on the $P_{T}$ of the dijet system could be useful to remove events with one or more badly measured jets. However, we find that the effects are small, and furthermore, biased towards higher dijet masses. We make no cut on dijet $P_{T}$. 
It has been suggested [ 13 ] that since the largest component of the QCD background is from gluon jets, variables which discriminate quark jets from gluon jets may be used. In the Monte Carlo, jets from gluon processes are significantly narrower than those in quark processes. However, it would be difficult to remove the $E_{T}$-dependence from any cut on jet shape. Furthermore, we see only small differences between the jets in samples expected to be quark-rich (such as photon + jet samples) and samples expected to be gluon-rich (such as dijet samples).

The cumulative acceptances of these cuts are listed in table 5.1 .

\begin{tabular}{||c|c|c||}
\hline & $W / Z$ eff. & BG eff. \\
\hline$E_{T}>12 \mathrm{GeV}$ & 0.89 & - \\
and $0.1<|\eta|<0.9$ & 0.18 & - \\
\hline Jet $3 E_{T}<12 \mathrm{GeV}$ & 0.83 & 0.68 \\
and $\Delta \phi_{j j}>160^{\circ}$ & 0.73 & 0.45 \\
\hline
\end{tabular}

Table 5.1: Efficiencies of kinematic cuts.

\subsubsection{W/Z signal expectation}

We now move on to studying the expected $W$ and $Z$ signal. Table 5.2 summarizes the expectations from Monte Carlo.

In $1.9 \mathrm{pb}^{-1}$ of usable data, we expect $2800 \mathrm{~W}$ events, and $1100 Z$ events. 


\begin{tabular}{||c|c|c||}
\hline & $W$ & $Z$ \\
\hline$\sigma_{\text {tot }}$ & $23.1 \mathrm{nb}$ & $6.86 \mathrm{nb}$ \\
$\sigma_{\text {had }}$ & $15.7 \mathrm{nb}$ & $4.80 \mathrm{nb}$ \\
kinematic cuts & $1.5 \mathrm{nb}$ & $640 \mathrm{pb}$ \\
Both jets match to quarks & $1.4 \mathrm{nb}$ & $580 \mathrm{pb}$ \\
\hline
\end{tabular}

Table 5.2: $\mathrm{W}$ and $\mathrm{Z}$ cross-sections after cuts.

\subsection{Measuring the Dijet Trigger Efficiency}

Different jet clustering algorithms are used in the Level 2 triggers (described in section 2.5.3) and final jet reconstruction (described in section 3.1.1). Level 2 clustering typically involves many fewer calorimeter towers, and registers less energy. A trigger cluster $E_{T}$ of $12 \mathrm{GeV}$ roughly corresponds to an uncorrected jet $E_{T}$ of $22 \mathrm{GeV}$, which in turn corresponds to a corrected jet $E_{T}$ of approximately $30 \mathrm{GeV}$.

To measure the efficiency of the dijet triggers, we will measure the efficiency for each jet to pass the dijet trigger threshold, as a function of raw jet transverse energy: We will then assume the efficiencies for both jets in the dijet trigger to be independent. so the trigger efficiency for a dijet event may be given by the product of the trigger efficiencies of the two jets. For these purposes, we calculate the $E_{T}$ not using the event vertex, but using the center of the detector $(z=0)$, to correspond more closely to the trigger $E_{T}$. These single-jet trigger efficiencies will be obtained from events in the inclusive jet data samples, which trigger if any Level 2 jet cluster $E_{T}$ is above a 
certain threshold.

We must ensure that the trigger requirements for the inclusive jet samples do not bias our measurement of central jet efficiency. To do this, we only consider dijet events with a trigger jet, a jet which could cause the inclusive jet trigger to fire, which lies outside the central region, and a probe jet, the one from which we will measure the efficiency, in the central region. We must require a trigger jet to be forward to eliminate trigger bias from the probe jet.

The forward-central technique uses inclusive jet data from the Run IB JET_20 and JET_j0 samples, and the Run 1C JET_15 sample. As a first check, we make sure the trigger efficiency from Run $1 \mathrm{~B}$ applies to Run $1 \mathrm{C}$ by comparing the efficiencies derived from the events in the Run $1 \mathrm{C}$ JET 15 sample which would pass the JET_20 requirements with the Run $1 B$ JET_20 sample. These are shown in figure 5.2 . No discrepancy is seen.

\subsubsection{Monte Carlo test of the method}

In figure 5.3, we test to see if the probe jets in the forward-central method have the same trigger turn-on curve as the jets selected in the dijet analysis. The agreement is good.

\subsubsection{Comparing efficiencies to dijet data}

We can study the trigger efficiency in dijet data by, for example, studying the probabilities, as a function of raw jet $E_{T}$, for jets with trigger $E_{T}$ of $10 \mathrm{GeV}$ to have a 


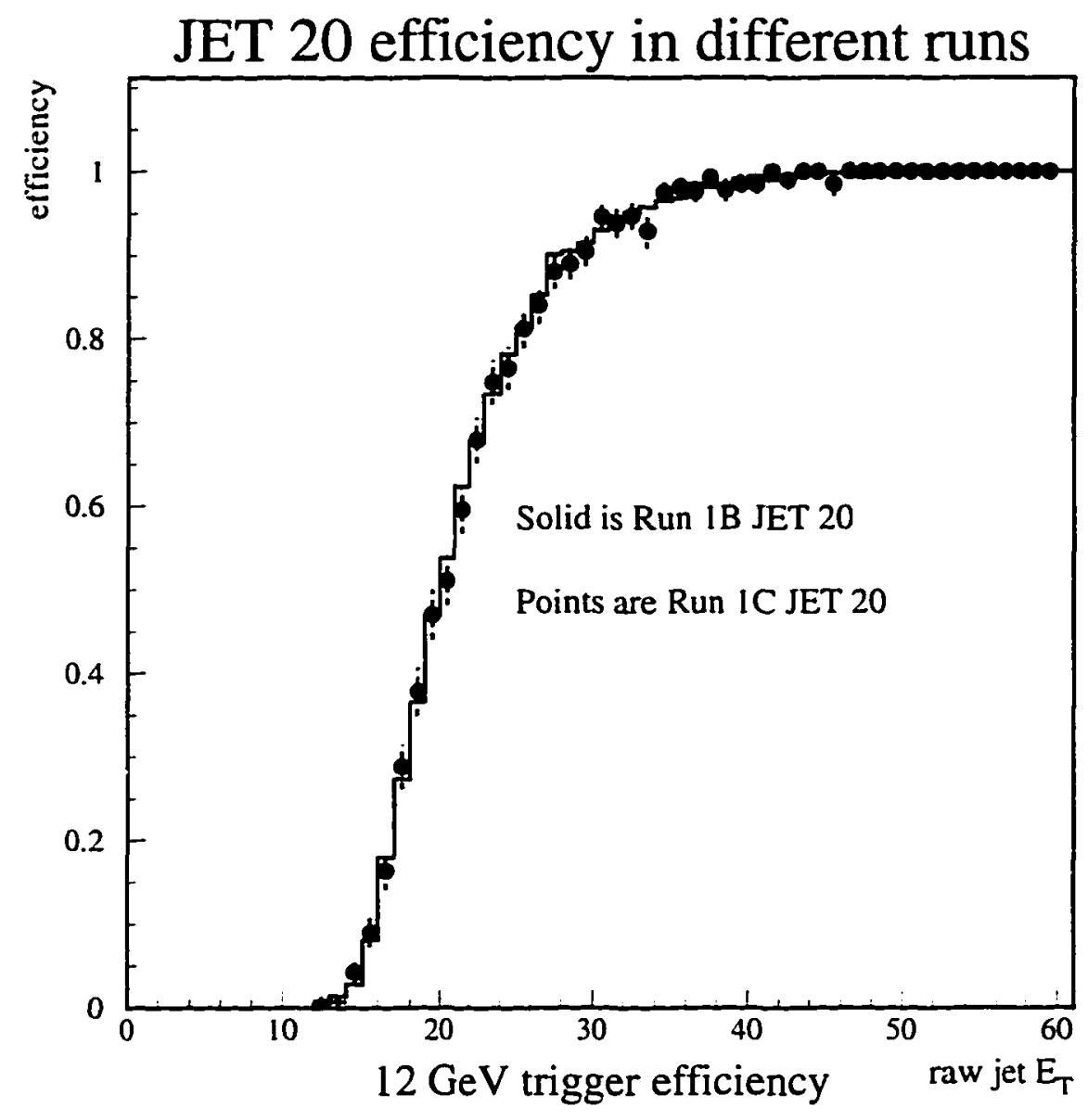

Figure 5.2: Comparing the $12 \mathrm{GeV}$ trigger efficiencies for JET_20 samples in runs 1B and $\mathrm{IC}$. 


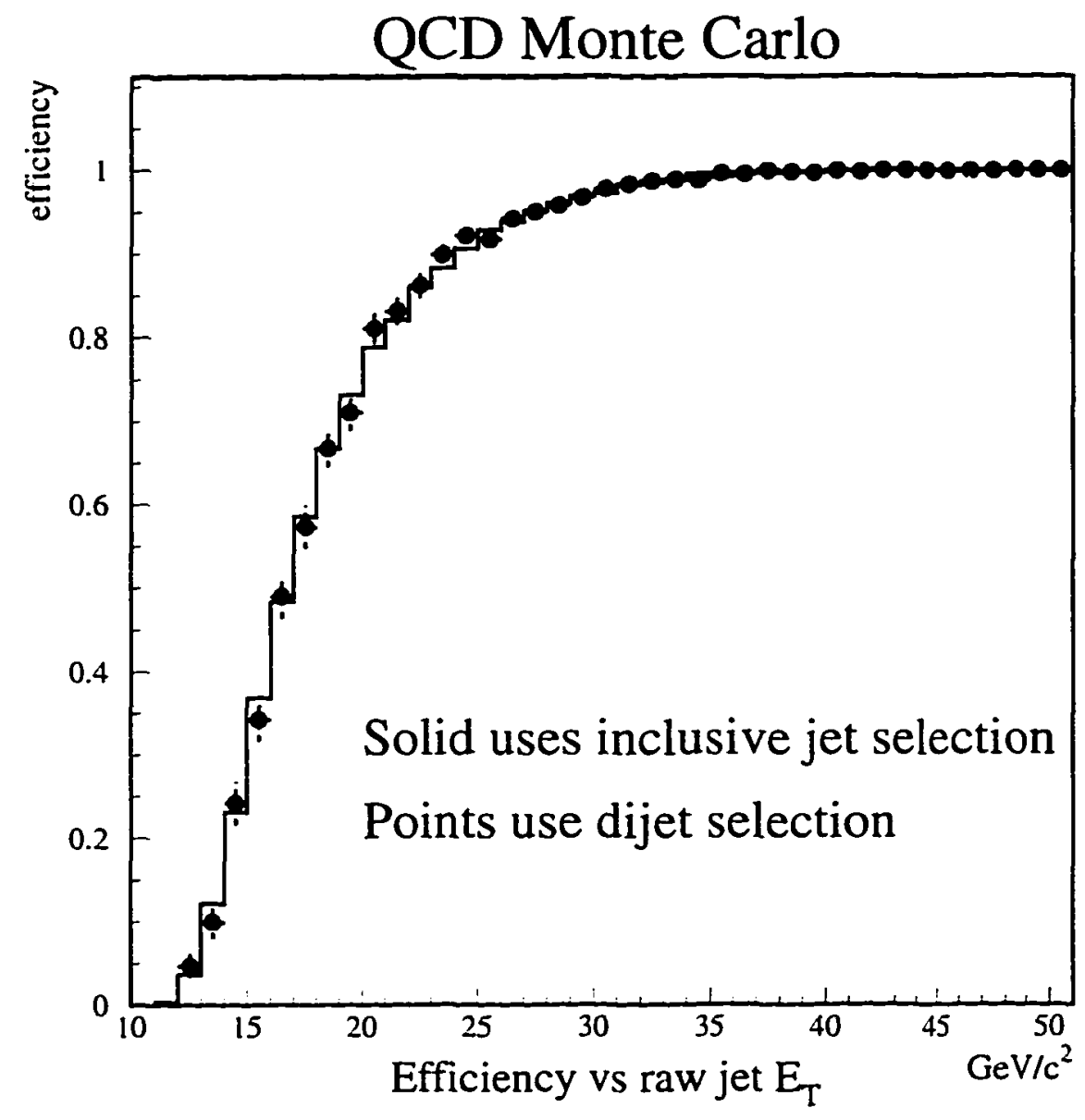

Figure 5.3: The Monte Carlo simulated trigger efficiencies for a jet to have a trigger $E_{T}$ of $12 \mathrm{GeV}$ or greater, for jets selected by both inclusive jet and dijet criteria. 
trigger $E_{T}$ of $12 \mathrm{GeV}$. In figure 5.4 , we plot the efficiencies for having trigger $E_{T}$ s of $12 \mathrm{GeV}$ given $10 \mathrm{GeV}$, and $15 \mathrm{GeV}$ given $12 \mathrm{GeV}$. The difference between the curves will be treated as a systematic uncertainty in section 5.6. The agreement is generally good.

\subsubsection{Multiple interactions}

Since the cone clustering algorithm typically involves more towers than the Level 2 algorithm, we expect that the contribution from additional soft $p \bar{p}$ interactions will be seen more in the cone algorithm. To test this, we plot in figure $\overline{5} . \overline{5}$ the efficiencies as a function of the number of high-quality $z$ vertices in the event. We see that extra interactions do give lower efficiencies. We will therefore apply separate efficiency curves for the cases of zero or one vertex, two vertices, and three vertices. We will reject events with four or more vertices.

We must also correct for an apparent decrease in vertex-finding in Run $1 \mathrm{C}$. The Run 1B JET_20 sample has more vertices per event than the Run 1C sample, vet a lower mean instantaneous luminosity. To account for this, we add random vertices to the dijet data, using a Poisson distribution of mean 0.2. After adding random vertices, $88 \%$ of the dijet events pass the requirement of three or fewer vertices.

\subsubsection{Eta dependence}

We also study the efficiencies in pseudorapidity bins of $0.1<|\eta|<0.3,0.3<|\eta|<0.5$, $0 . \bar{j}<|\eta|<0.7$, and $0.7<|\eta|<0.9$ in figure 5.6. We see that efficiency falls off at 

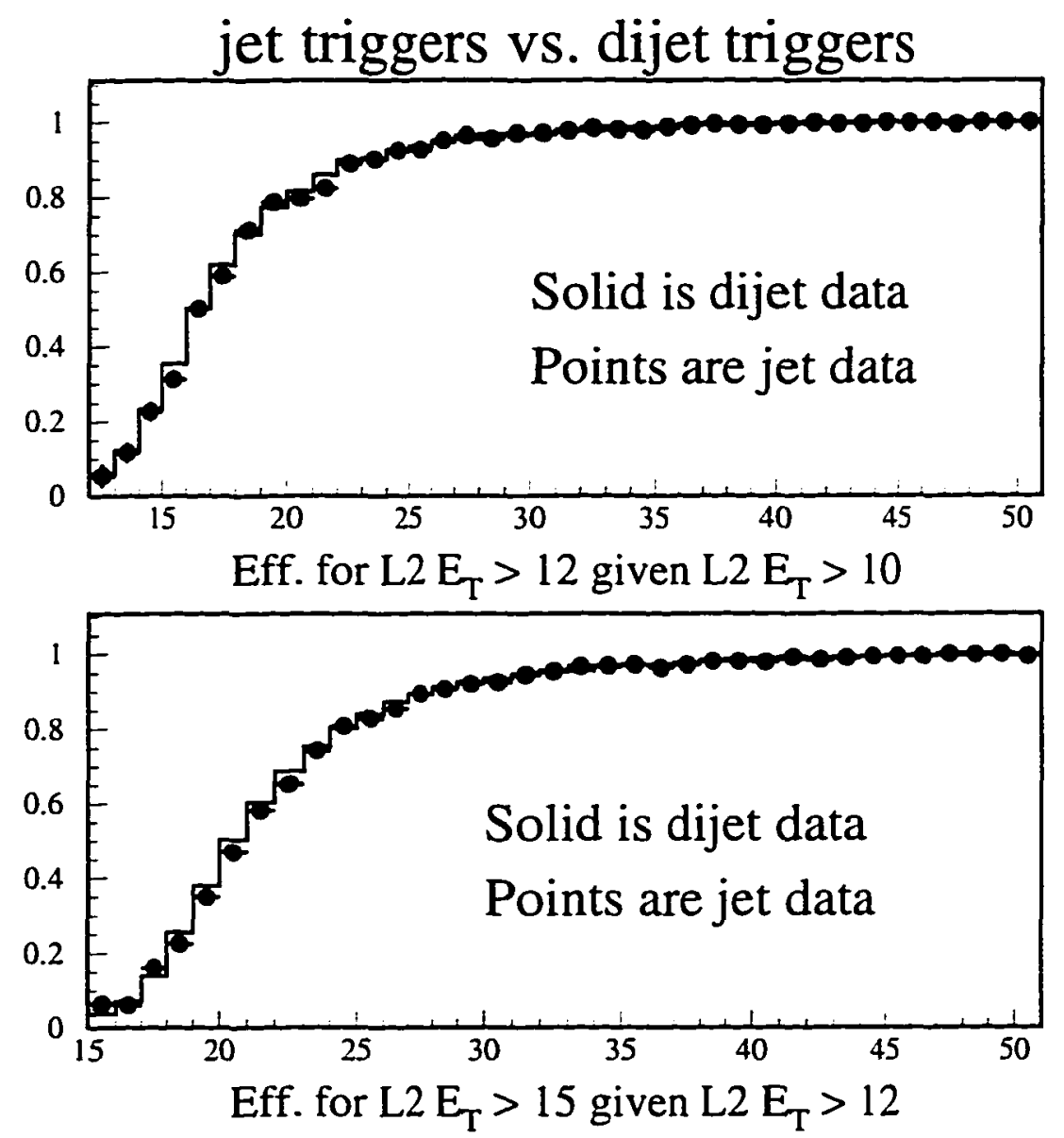

Figure 5.4: The top plot compares the efficiency for jets to have a trigger $E_{T}$ greater than $12 \mathrm{GeV}$, given that the trigger $E_{T}$ is already greater than $10 \mathrm{GeV}$, for DIJET_10 and inclusive jet data. The bottom plot compares the efficiency for having $15 \mathrm{Gel}^{\text {. }}$ given $12 \mathrm{GeV}$, using the DIJET_12 and inclusive jet samples. 


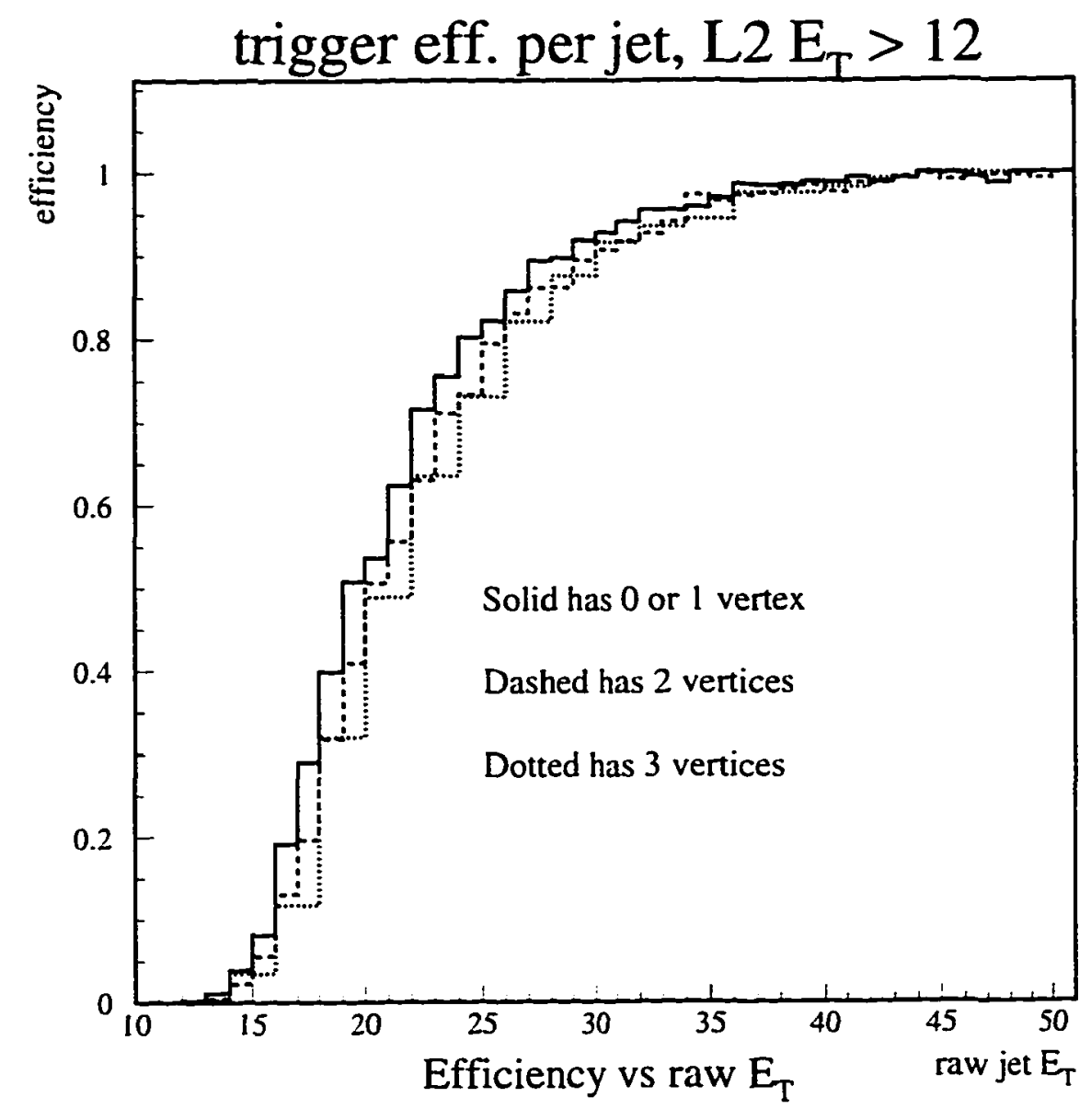

Figure 5.5: The dependence of the efficiency curves on the number of event vertices in the event. 
higher $\eta$. We therefore compute separate efficiencies for the cases $0.1<|\eta|<0 . \overline{5}$. $0.5<|\eta|<0.7$, and $0.7<|\eta|<0.9$.

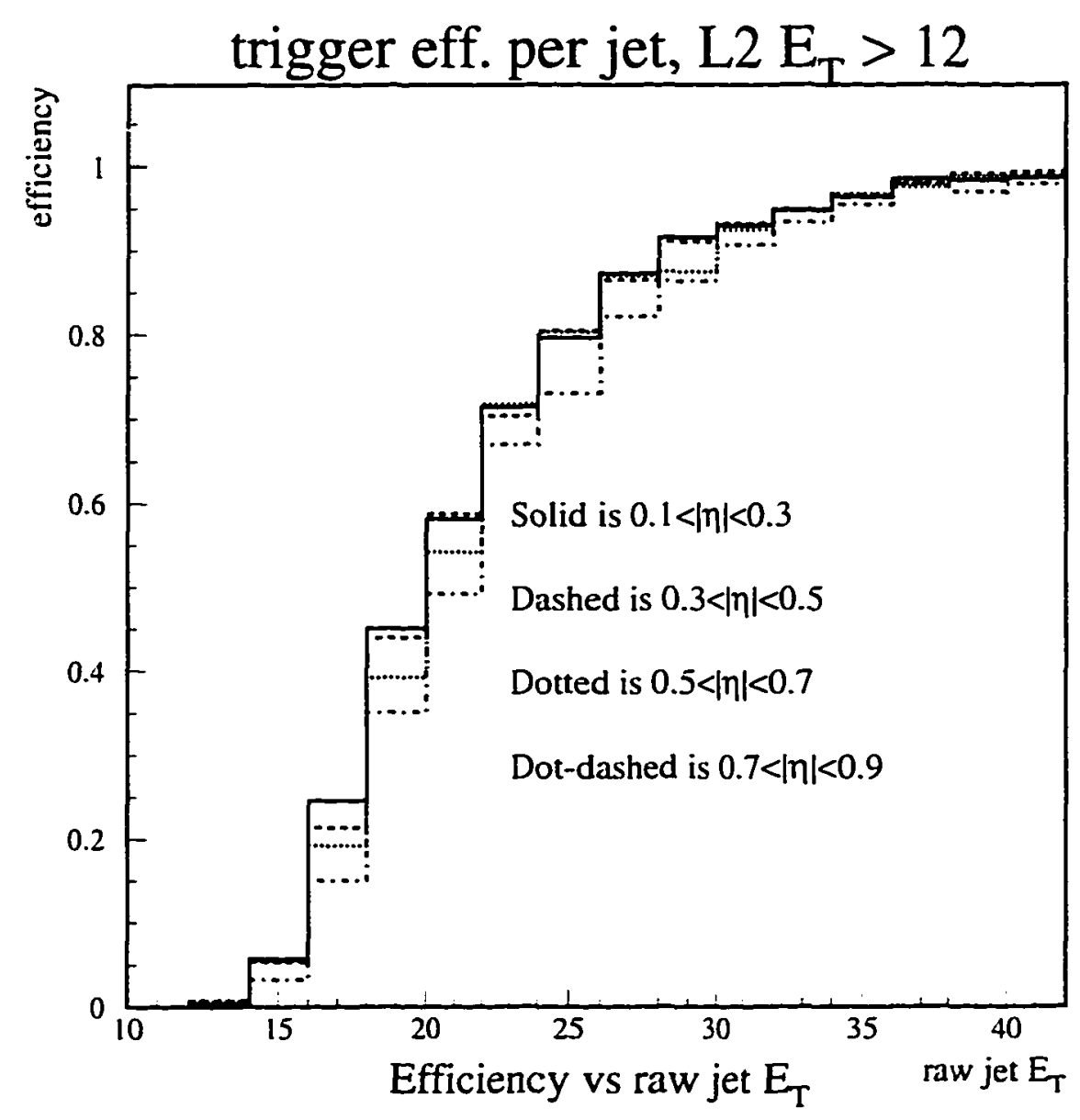

Figure 5.6: The dependence of the efficiency curves on jet pseudorapidity.

\subsubsection{Final trigger efficiency curves}

Combining the pseudorapidity bins with the multiple-interaction binning, we obtain nine separate efficiency plots. They are shown in figure 5.7. These plots are made 
into smooth curves before they are applied to dijet data.
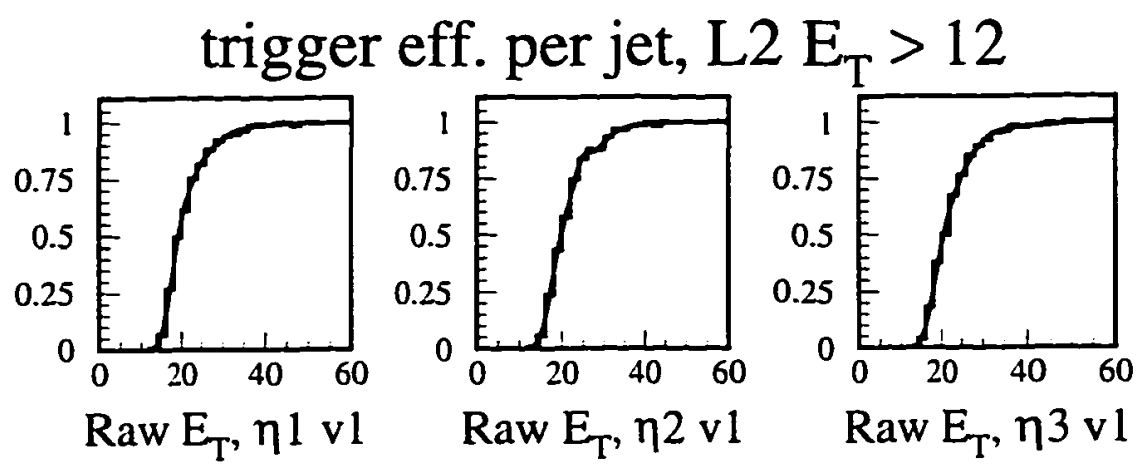

Raw $\mathrm{E}_{\mathrm{T}}, \eta 2 \mathrm{vl}$

Raw $E_{\mathrm{T}}, \eta 3 \mathrm{vl}$
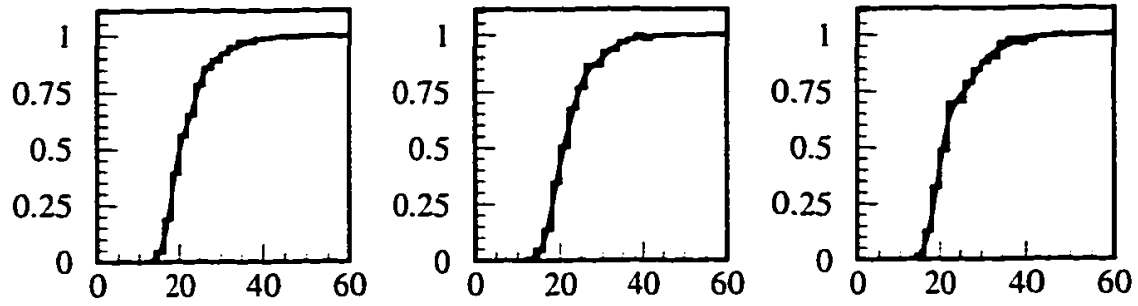

Raw $E_{\mathrm{T}}, \eta 1 \mathrm{v} 2$

Raw $\mathrm{E}_{\mathrm{T}}, \eta 2$ v2

Raw $E_{\mathrm{T}}, \eta 3 \mathrm{v} 2$
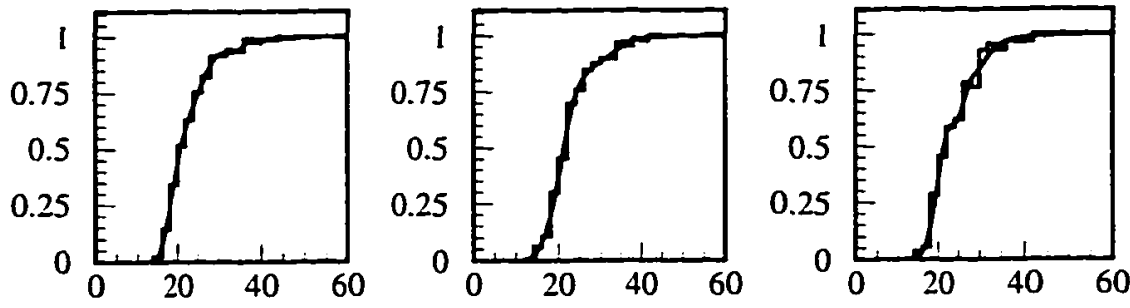

Raw $E_{\mathrm{T}}, \eta 1 \mathrm{v} 3$

Raw $E_{T}, \eta 2$ v3

Raw $E_{T}, \eta 3$ v3

Figure 5.7: Trigger efficiency, as a function of raw jet $E_{T}$, for the bins in number of vertices and pseudorapidity. The three $\eta$ bins correspond to $0.1<|\eta|<0.5$, $0 . \overline{\mathrm{j}}<|\eta|<0.7$, and $0.7<|\eta|<0.9$.

\subsubsection{Correcting for jet $E_{T}$ cuts}

We still need one more correction before we can claim an unbiased spectrum; we must correct away the effects of the $E_{T}$ cuts on the jets. To do this, we turn to PYTHI. 
dijet Monte Carlo, using the same cuts as the dijet analysis. We ask. as a function of uncorrected dijet mass, how often both uncorrected jet $E_{T}$ 's pass the threshold.

This method also gives us the option of changing the jet $E_{T}$ cut. We will move the cut up to $15 \mathrm{GeV}$, in order to avoid events with large weights from trigger efficiency corrections. We will still use the cut of $12 \mathrm{GeV}$ as a cross-check.

The efficiencies of raw jet $E_{T}$ cuts of 12 and $15 \mathrm{GeV}$ are shown as a function of corrected dijet mass in figure 5.8 .

\subsection{Analysis of Dijet Data}

In this section, we use the single jet efficiencies obtained in the previous section to correct for inefficiency of the dijet trigger. To do this, we will weight each dijet event seen by the inverse of the trigger efficiency for each of its jets. We will also weight the event by the inverse of the efficiency of the jet $E_{T}$ cut. Because we use this weighting technique, our expected number of signal events should not depend on trigger efficiency, assuming the signal jets are no more likely to trigger than background jets.

The dijet mass spectrum, with and without trigger corrections, is shown in figure 5.9. A raw jet $E_{T}$ cut of $15 \mathrm{GeV}$ is used, and corrections are made as functions of $\eta$ and the number of event vertices. After all the cuts, we are left with 841.405 events. The trigger efficiency curve, as a function of corrected dijet mass, is also shown. We note, with some dismay, that the efficiency at $80 \mathrm{GeV} / c^{2}$ is only $70 \%$. and at $60 \mathrm{GeV} / c^{2}$ drops to about $20 \%$. 


\subsubsection{Fits}

Once we have the corrections, we weight each dijet event by the product of the inverses of the efficiencies for each of the two jets, and by the inverse of the efficiency of the jet $E_{T}$ threshold. The resulting dijet spectrum is fit to the following functional form. used by UA2 [41].

$$
A m^{-B} e^{-C m} e^{-D m^{2}}
$$

We also add a signal term to the fit. We use Gaussians to represent the $W$ and $Z$ components. The width of the $W$ signal is taken from Monte Carlo to be 10.9 $\mathrm{GeV} / c^{2}$, and the $Z$ signal to be $11.4 \mathrm{GeV} / c^{2}$. The ratio of $Z$ to $W$ events is fixed to be 0.41 . The masses are allowed to float, but the ratio of their masses is fixed to 1.134.

Allowing this, the fit gives a good $\chi^{2}$ down to a mass of $55 \mathrm{GeV} / c^{2}$. However, the fit did not find any signal, and actually preferred a slight dip in the data. Figure 5.10 plots the difference between the data and fitted background shape.

\subsection{Toy Monte Carlo studies}

This analysis thus far combines a steeply falling dijet spectrum with a steeply rising trigger turn-on. It seems reasonable that errors in the trigger efficiency curves could result in fake bumps. To study the size of these effects, we use a toy Monte Carlo approach. We create "true" data points according to the shape fitted from the fully 


\begin{tabular}{||c|c|c||}
\hline Variation & Fitted $W$ mass & \# of $W$ s and $Z$ 's fitted \\
\hline jet $E_{T}$ cut $12 \mathrm{GeV}$ & 75.8 & 2600 \\
12-given-10 discrepancy & 70.6 & -7500 \\
15-given-12 discrepancy & 83.4 & 2750 \\
Add 0.3 extra vertices & 76.2 & 4400 \\
Add 0.1 extra vertices & 80.0 & 100 \\
\hline
\end{tabular}

Table 5.3: Magnitudes of the fake bumps produces by varying the trigger efficiency corrections. The expected signal is about 4000 events.

corrected data, without allowing statistical fluctuations. We then apply a trigger efficiency, and use variations to that trigger efficiency to correct the data. We then fit to a signal plus background. There are many variations in trigger efficiencies we may use. We may use different jet $E_{T}$ cutoffs. We may change the jet efficiencies by the amount of the discrepancy found in section 5.4.2. We also investigate the effects of adding more or fewer extra vertices.

We find that many of these effects produce fake bumps, often much larger than the expected signal of $\approx 4000$ events. One such effect is shown in figure 5.11 , for the case of shifting the efficiency by the discrepancy in Fig. 5.4a. Although we see the variation in the correction to be small, it creates a large fake bump in the data.

The magnitude of the fake bumps seen by variations on the trigger efficiency are shown in table 5.3 . 


\section{7 $\quad b$ tagging results}

In this section, we seek evidence for the process $Z \rightarrow b \bar{b}$. This signal would be valuable to set the jet energy scale for $b$ jets for the top quark mass measurement.

Tagging pairs of heavy flavor jets is difficult in the dijet sample, because half of the CTC only had three operational superlayers. Because of this, it seems the best way to find the signal would be to look in the single SECVTX-tag channel. To find the background shape, we apply the SECVTX fake rate parametrization to the data, and give it a smooth fit using the function in equation 5.1. We then divide the SECVTXtagged data by this background shape. The result is shown in figure 5.12. No excess is visible around the $Z$ mass. Applying fit techniques from previous sections also fails to find a signal.

It seems possible that the CTC conditions could degrade the SECVTX tagging rate on the side of the detector where the CTC is on, since moderately displaced tracks may affect the primary vertex fit. However, the tagging rates in the JET_20 samples from Runs $1 B$ and $1 C$ agree reasonably well.

Although double-tags are rare because of the CTC conditions, we present in figure 5.13 the dijet mass plots for events which have either two SECVTX tags, or one SECVTX tag and another soft lepton tag or jet probability of less than $5 \%$. Again, no signal is seen. 


\subsection{Future trigger requirements}

Assuming the same signal-to-noise ratio as with current cuts, we present in table 5.4 the statistical precision of the $W$ mass obtained from ensembles of pseudo-experiments performed with various sample sizes. Clearly, this measurement of the $W$ mass could never rival that of the leptonic $W$ modes, but the potential for study of the jet energy scale is good.

\begin{tabular}{||c|c|c|c||}
\hline$N_{W}$ & luminosity needed $\left(\mathrm{pb}^{-1}\right)$ & $\operatorname{mass}\left(\mathrm{GeV} / \mathrm{c}^{2}\right)$ & Fitted $N_{W}$ \\
\hline 5000 & 2.7 & $80.5 \pm 3.1$ & $4260 \pm 850$ \\
10000 & 5.3 & $80.8 \pm 1.9$ & $8200 \pm 1600$ \\
25000 & 13.3 & $80.5 \pm 1.3$ & $20900 \pm 2700$ \\
50000 & 26.7 & $80.4 \pm 1.0$ & $41900 \pm 3600$ \\
\hline
\end{tabular}

Table 5.4: Results of ensembles of 500 dijet pseudo-experiments. The input mass was $80.33 \mathrm{GeV} / c^{2}$.

To see hadronic $W$ decays, we need a trigger with high efficiency down to a dijet mass of $60 \mathrm{GeV} / c^{2}$. Since any such trigger will be limited by rate, we must choose it so it triggers with the highest possible signal-to-noise ratio, and the lowest possible acceptance for events with dijet masses below $50 \mathrm{GeV} / c^{2}$. We will limit ourselves to central dijet pairs, with $0.1<|\eta|<0.7$. The softer of the two jets sometimes has no Level 2 cluster, so we should start with a single jet trigger $E_{T}$ of $8 \mathrm{GeV}$ and $|\eta|<0.8$. To cut away the tail at low dijet masses, we should also require that the summed 
calorimeter $E_{T}$ be less than $25 \mathrm{GeV}$. The efficiency for these trigger requirements is obtained from dijet pairs from low $P_{T}$ muon triggers, and is shown in figure 5.14 .

The rates of these triggers can be calculated from minimum-bias datasets. The acceptance for minimum bias events is found to be $0.03 \%$. For a minimum-bias cross section of $50 \mathrm{mb}$ and luminosities of $10^{32} \mathrm{~cm}^{-2} \mathrm{~s}^{-1}$, this trigger will pass events through Level 2 at a rate of $1500 \mathrm{~Hz}$, which is unacceptably high. The maximum Level 2 accept rate for Run II is $300 \mathrm{~Hz}$, and a calibration trigger should require no more than a few percent of this. Prescaling the triggers would reduce this rate, but the prescale factor needed would make the $W$ mass precision obtained through this method lower than the precision obtained from a future double $b$-tagged $t \bar{t}$ analysis. 


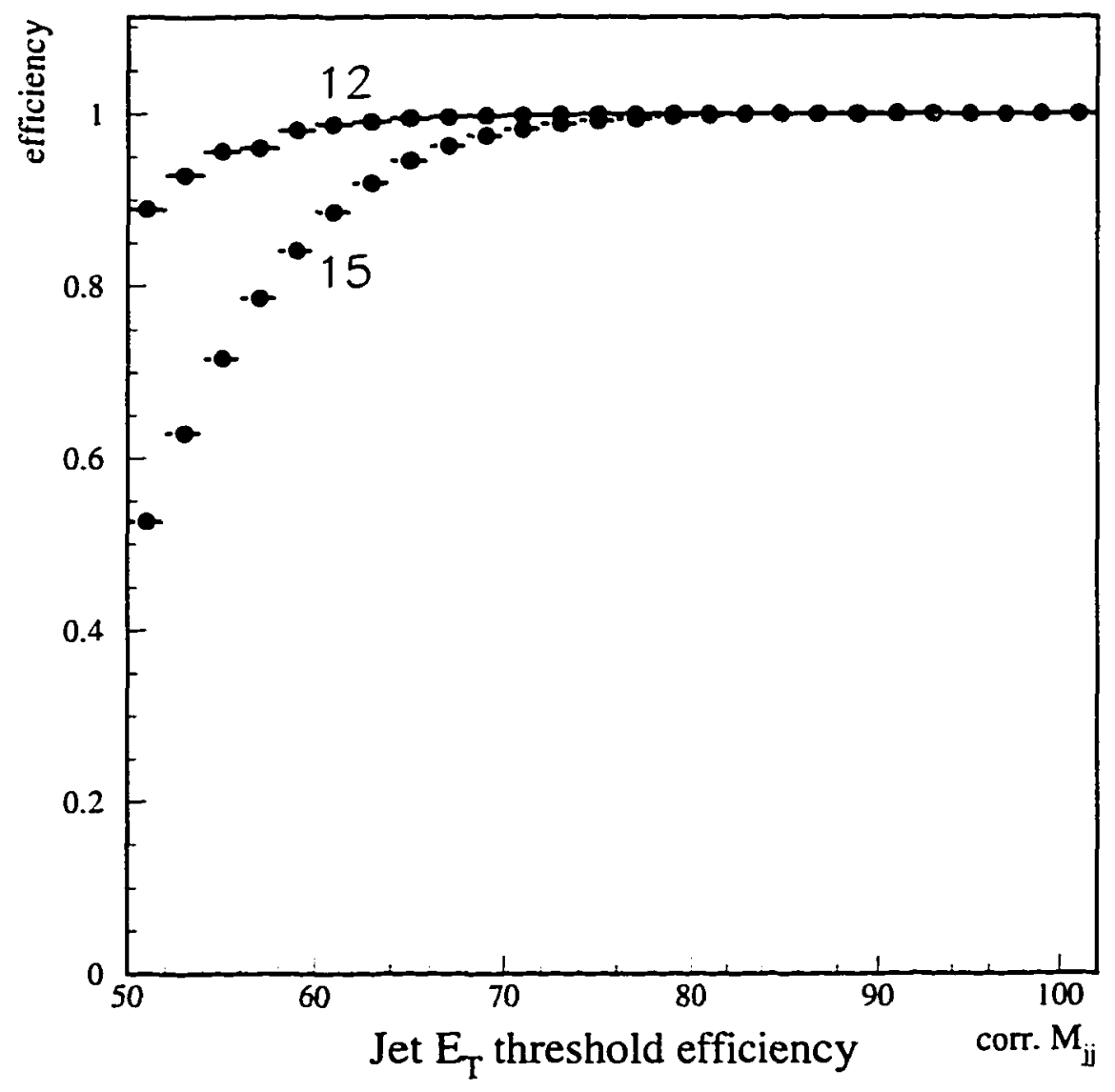

Figure 5.8: The efficiency of the raw jet $E_{T}$ thresholds of 12 and $15 \mathrm{GeV}$, as a function of corrected dijet mass. A correction is applied to the dijet mass spectrum. as a function of the raw dijet masses. 

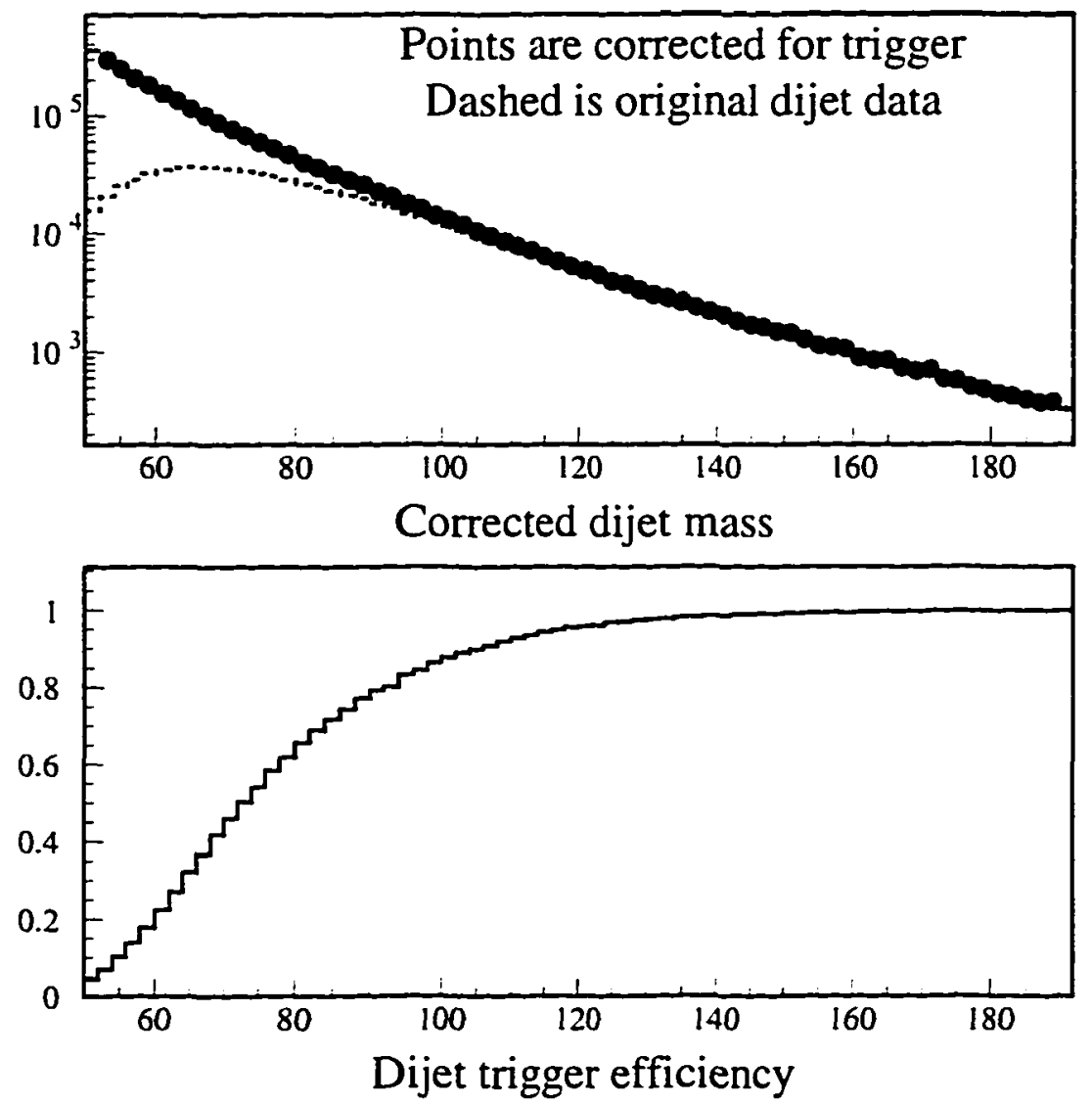

Figure 5.9: The top plot shows the dijet mass spectrum for DIJET_12 triggers, with and without trigger efficiency corrections. The bottom plot is the trigger efficiency. 


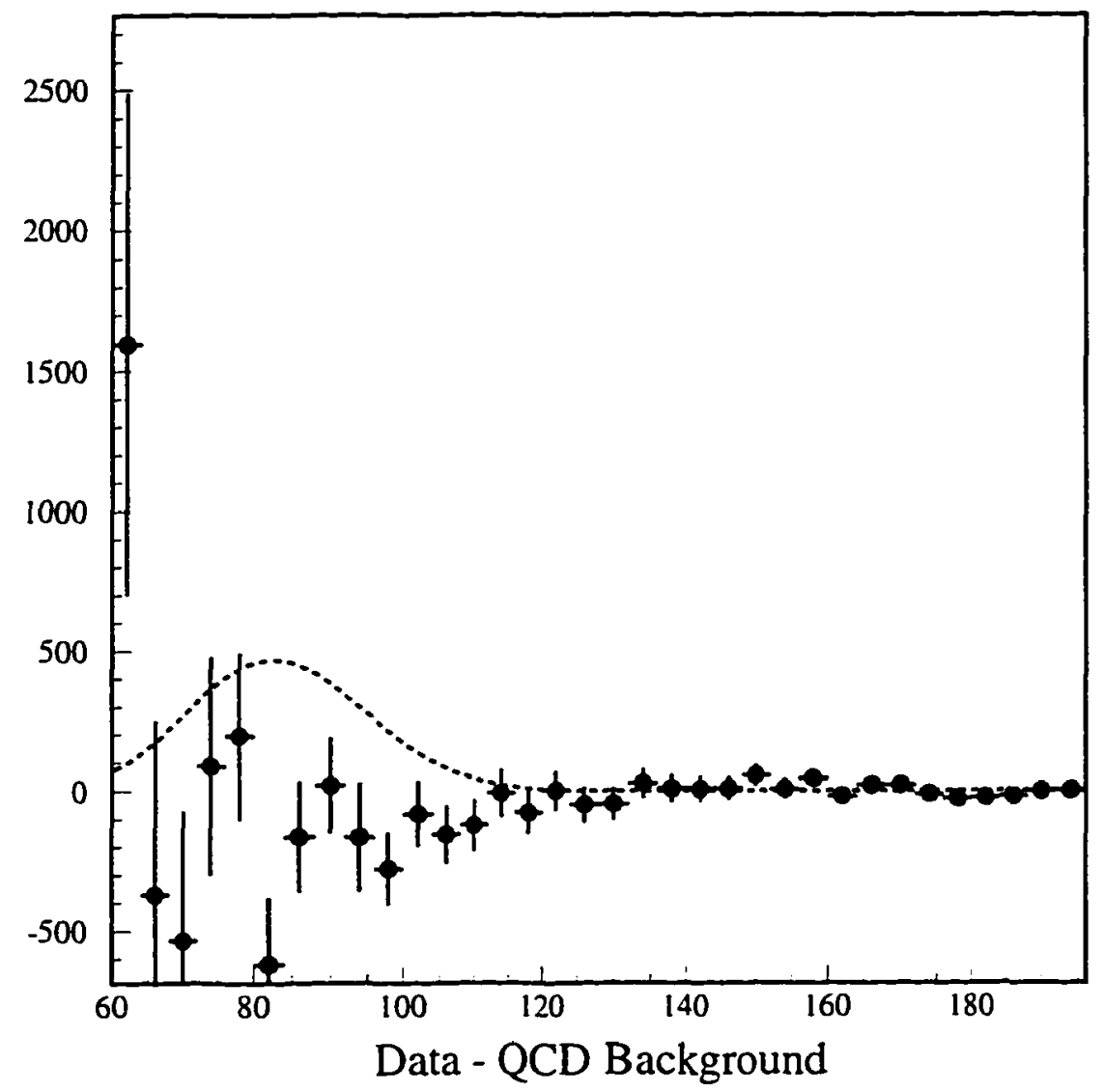

Figure 5.10: The difference between the data and fitted background. No signal is seen. 

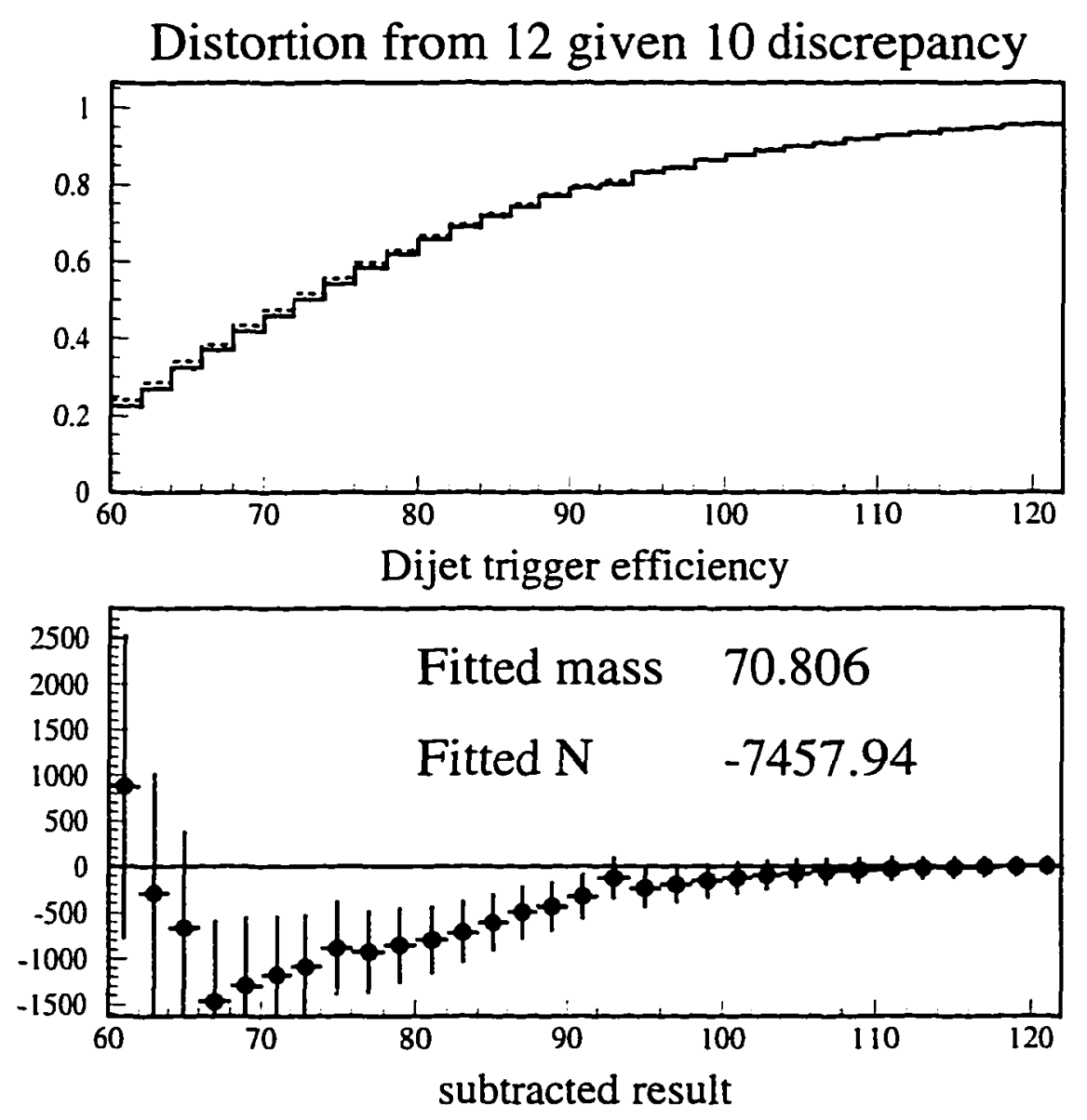

Figure 5.11: Applying a trigger efficiency that is incorrect by a small amount leads to a fake bump in a smooth dijet mass spectrum. The variation comes from shifting the single-jet trigger efficiency by the amount of the discrepancy in Fig. 5.4a. 


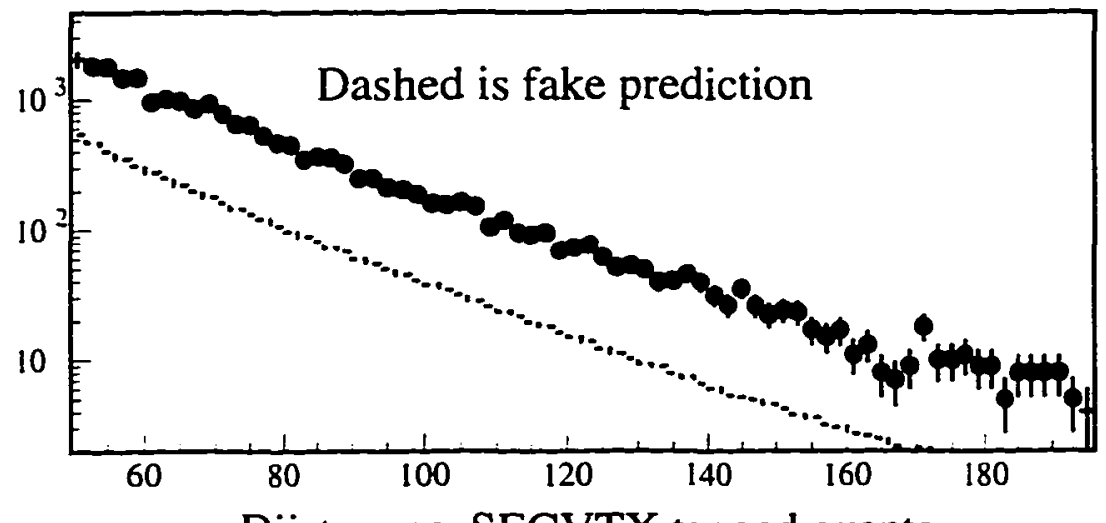

Dijet mass, SECVTX tagged events

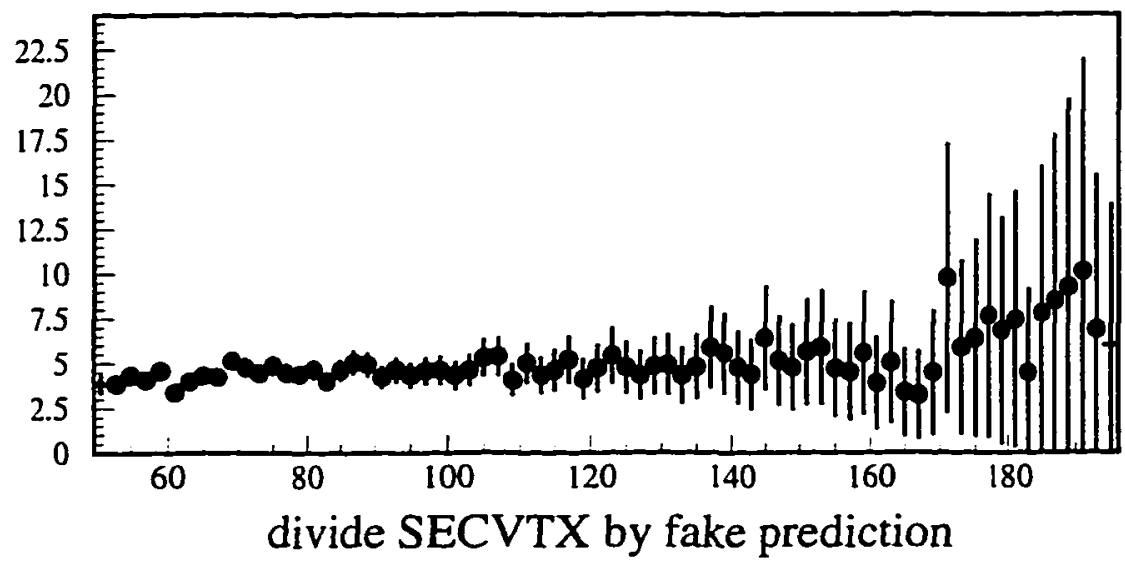

Figure 5.12: The SECVTX tagged data and fake rates. The bottom plot divides the data by the fake rate. All entries are weighted by their DIJET_12 trigger efficiency. 

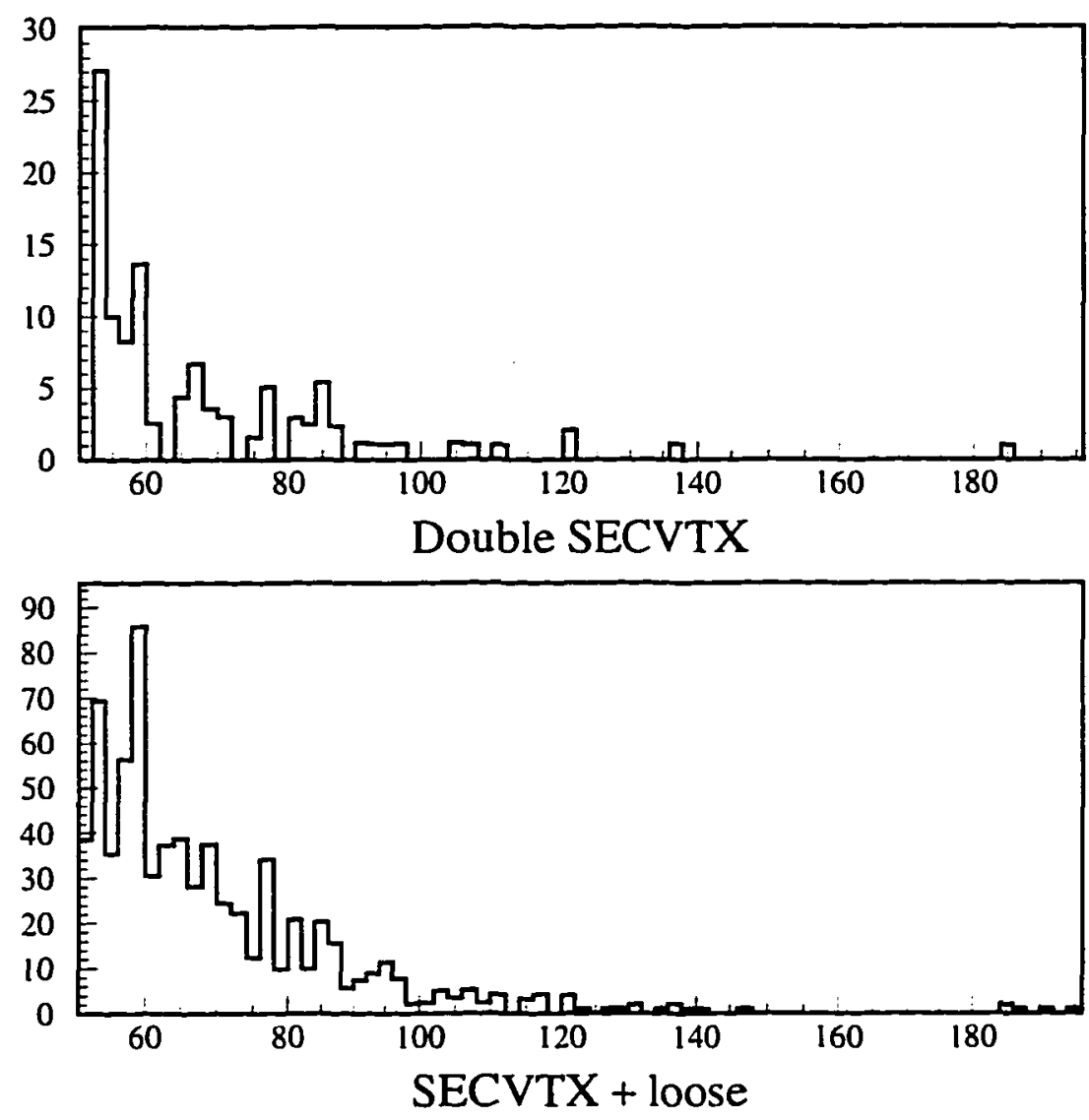

Figure 5.13: The top plot shows the corrected dijet mass spectrum for events with two SECVTX tags. The bottom shows events with one SECVTX tag, and one tag of either SECVTX, SLT, or Jet Probability < $5 \%$. 


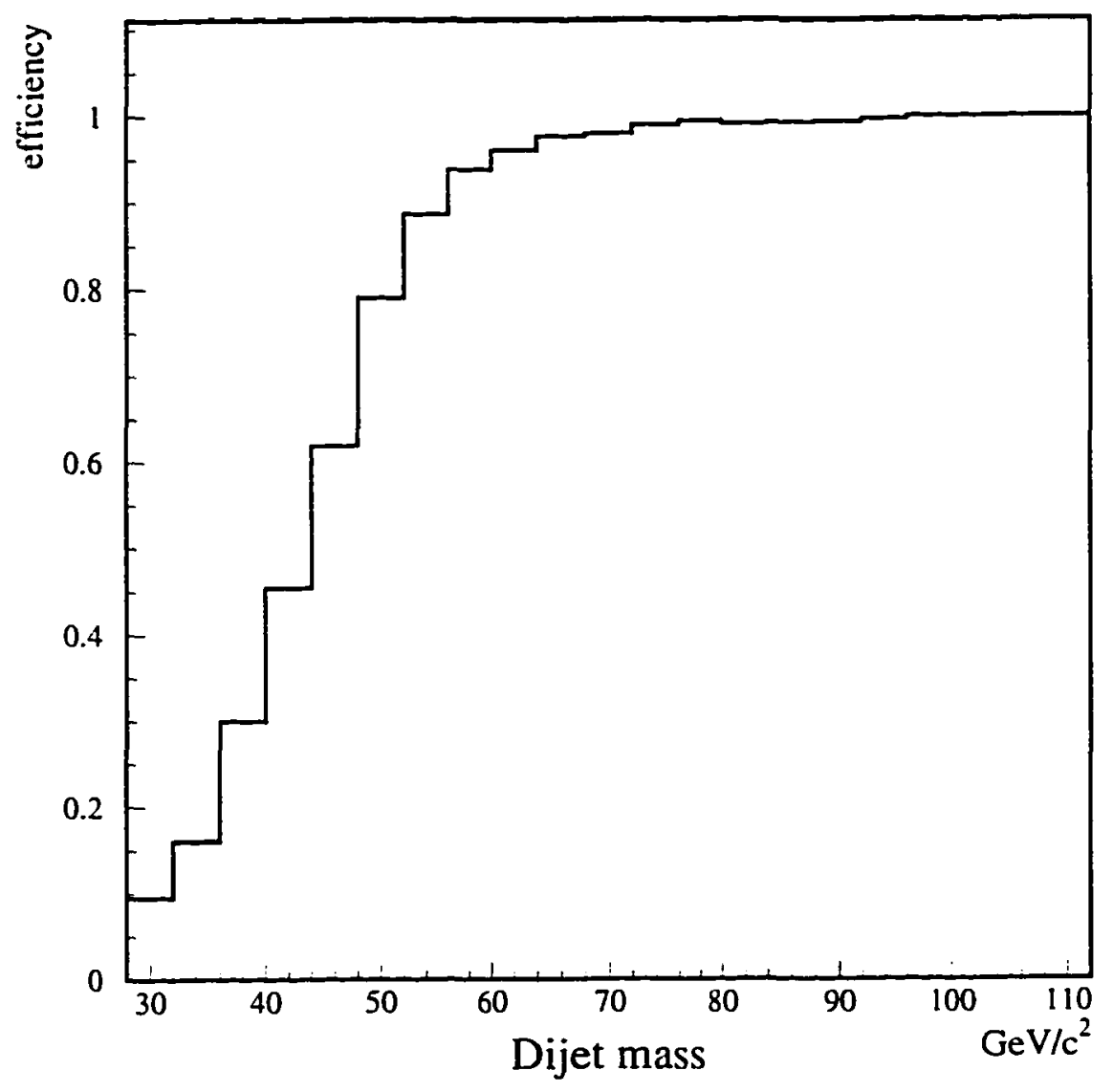

Figure 5.14: Trigger efficiency for a Level 2 trigger with $E_{T}>8 \mathrm{GeV},|\eta|<0.8$, and $\Sigma E_{T}>25 \mathrm{GeV}$, obtained from the low $P_{T}$ muon sample. 


\section{Chapter 6}

\section{$W^{+} W^{-}$production}

Another mode in which the hadronic decays of $W$ and $Z$ bosons may be observed is in boson pair production, where the final states may be $W W, W Z$, or $Z Z$. The Standard Model NLO cross sections for these processes are shown below $[44,45]$. $W^{+} W^{-}$production has been observed the dilepton mode at CDF [46]. Also, in June 1996 the LEP II $e^{+} e^{-}$collider at CERN began running at sufficient center-of-mass energies to produce $W^{+} W^{-}$.

$$
\begin{gathered}
\sigma(W W)=(9.5 \pm 0.7) p b \\
\sigma(W Z)=(2.6 \pm 0.34) p b \\
\sigma(Z Z)=(1.0 \pm 0.2) p b
\end{gathered}
$$

Diboson processes provide opportunities to test the Standard Model predictions of $W W Z$ and $W W \gamma$ gauge couplings. Delicate cancellations occur in the Standard Model to prevent $W W$ processes from violating unitarity. Almost any deviation from Standard .Model couplings results in larger cross-sections, with the increase concentrated at high $\sqrt{\hat{s}}$. Limits on anomalous triboson couplings have been set at 
CDF [4T] and D0 [48] based on searches for very high- $p_{T} W W^{-}$processes in the lepton plus jets mode. New phenomena, such as Higgs bosons, heavy quarks and leptons, or technicolor, may also result in enhanced $W W$ production $[49,50]$.

Observation of $W W$ or $W Z$ processes in the $W+2$ jets mode is difficult, because the backgrounds from $W+\mathrm{QCD}$ processes are large. The dijet mass spectrum for the CDF $W+2$ jet sample is shown in figure 6.1 , along with the predicted signal of ( 40 \pm 12 ) events. Furthermore, we have found no kinematic cuts effective in increasing the significance of the signal $S / \sqrt{N}$, having tried jet $E_{T}, \eta, W P_{T}, \cos \theta^{*}$ of the dijet system, and jet separation. A $\cos \theta^{*}$ cut of 0.6 , however, may be useful in reducing $S / N$ at constant signal significance, and cutting on jet $E_{T}$ will decrease the width of the signal peak. In the future, the observation depends not on statistics, but on our ability to model the kinematics of the $W+$ QCD background. Discovery of this mode may be possible with $2 \mathrm{fb}^{-1}$ of Run II data. 


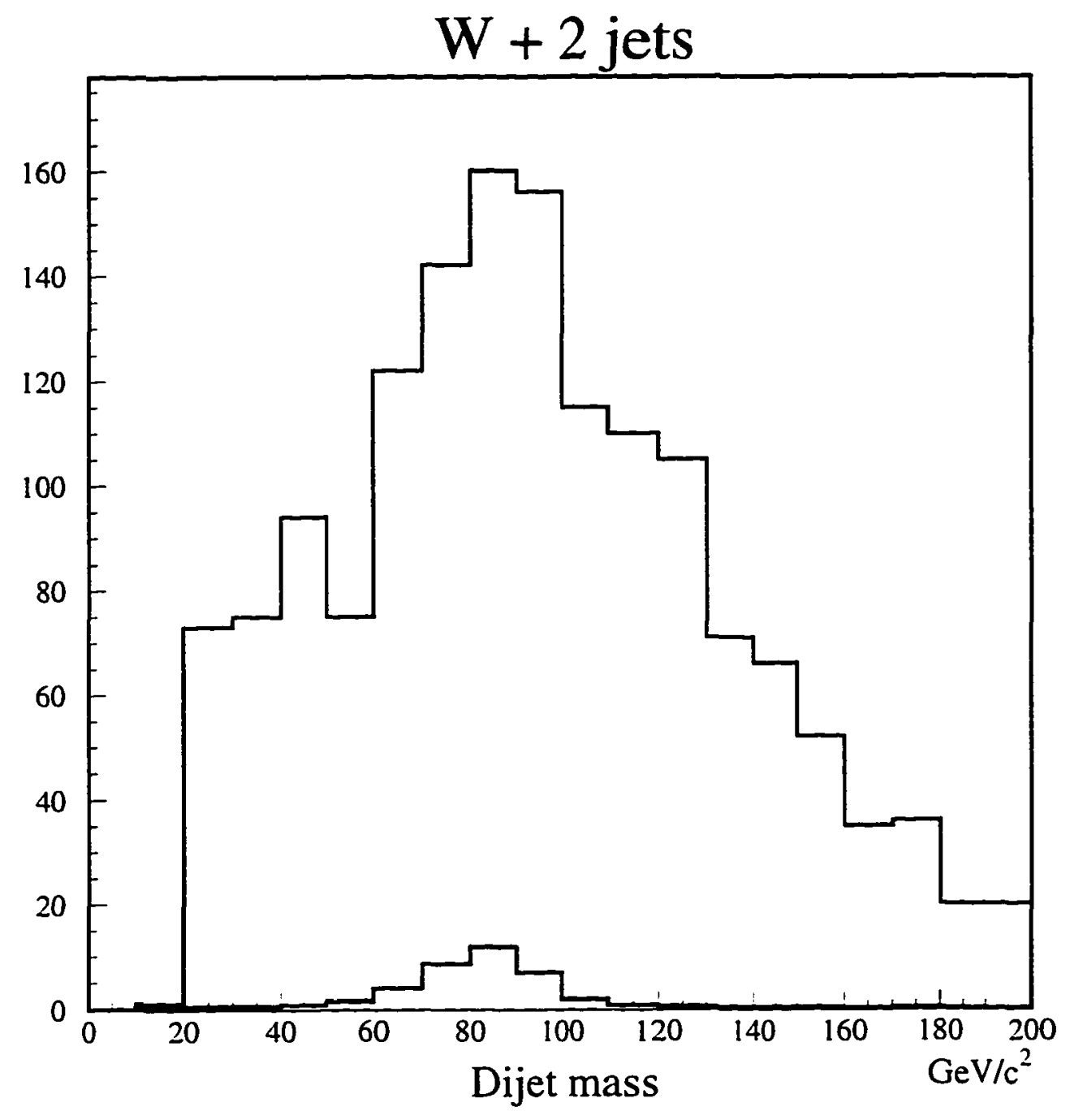

Figure 6.1: The dijet mass spectrum for the CDF $W+2$ jet sample, with the expected $W W$ and $W Z$ contribution superimposed. 


\section{Chapter 7}

\section{Conclusion}

Evidence has been presented for the hadronic decay mode of the $W$ boson in $t \bar{t}$ events at CDF. Evidence for this process has also been seen at CDF in without the use of $b$-tagging [39], and by top mass reconstruction [51]. Combining these results gives a significance of $3.3 \sigma$ over the predicted background shape [52]. This result demonstrates the presence of a second $W$ boson in semileptonic $t \bar{t}$ candidates.

The technique used to isolate this peak, loose double $b$-tagging, has also been seen to be valuable [53] for future searches for Higgs bosons decaying to $b \bar{b}$.

While no evidence was seen for direct $W$ and $Z$ production in the dijet sample, techniques were developed to measure and correct for trigger inefficiency. Recently, it has been suggested [54] that the scaling of CDF's inclusive jet spectra taken at $\sqrt{s}=630$ and $1800 \mathrm{GeV}$ allows the possibility of squarks of mass $100-200 \mathrm{GeV}$. which then decay into quark-light gluino pairs. The dijet dataset may be useful in searching for this process.

For Run II, scheduled to begin in 1999, the $b$-tagging abilities of the CDF detector will be greatly improved [55]. A longer, five-layer SVX will provide three-dimensional 
tracking. Combined with a new inner tracker, it will be possible to trigger on secondary vertices, greatly aiding the search for $Z \rightarrow b \bar{b}$. Improved tracking and muon coverage will increase lepton acceptance. With these improvements, we expect to tag $b$ jets at a rate of $65 \%$ [56]. From this, we expect 258 double $b$-tagged $t \bar{t}$ events per $\mathrm{fb}^{-1}$ of data, even without any looser second tagging. With higher statistics, it may be possible to further "clean up" the $W+\geq 4$ jet sample, by making requirements on the absence of a fifth jet, looser dilepton removal cuts, or by requiring the SLT track to be displaced. Double $b$-tagged $t \bar{t}$ events will undoubtedly be a major tool in the study of the top quark. As stated in Fermilab's "TeV2000 Report, Top Physics at the Tevatron":

... the identifiable hadronic $W$ decays (in double $b$-tagged) $t \bar{t}$ events will be a laboratory for study of jet and Monte Carlo modelling. The double tag sample may be the sample of choice for the ultimate top mass analysis, with better control of systematic effects than present studies can anticipate. 


\section{Appendix A}

\section{The CDF Collaboration}

The CDF collaboration consists of over 450 physicists from 39 institutions in seven countries. The members of the CDF collaboration in Run $1 \mathrm{~B}$ are listed below.

F. Abe, ${ }^{17}$ H. Akimoto, ${ }^{36}$ A. Akopian, ${ }^{31}$ M. G. Albrow, ${ }^{7}$ S. R. Amendolia. ${ }^{27}$ D. Amidei, ${ }^{20}$ J. Antos, ${ }^{33} \mathrm{~S}$. Aota, ${ }^{36} \mathrm{G}$. Apollinari, ${ }^{31} \mathrm{~T}$. Asakawa, ${ }^{36} \mathrm{~W}$. Ashmanskas, ${ }^{18}$ M. Atac, ${ }^{7}$ F. Azfar, ${ }^{26}$ P. Azzi-Bacchetta, ${ }^{25}$ N. Bacchetta, ${ }^{25}$ W. Badgett,${ }^{20}$ S. Bagdasarov, ${ }^{31}$ M. W. Bailey, ${ }^{22}$ J. Bao, ${ }^{39}$ P. de Barbaro, ${ }^{30}$ A. BarbaroGaltieri, ${ }^{18}$ V. E. Barnes, ${ }^{29}$ B. A. Barnett,${ }^{15}$ M. Barone,${ }^{9}$ E. Barzi, ${ }^{9}$ G. Bauer ${ }^{19}$ T. Baumann, ${ }^{11}$ F. Bedeschi, ${ }^{27}$ S. Behrends, ${ }^{3}$ S. Belforte,${ }^{27} \dot{\mathrm{G}}$. Bellettini, ${ }^{27}$ J. Bellinger, ${ }^{38}$ D. Benjamin, ${ }^{35}$ J. Benlloch, ${ }^{19}$ J. Bensinger, ${ }^{3}$ D. Benton, ${ }^{26}$ A. Beretvas, ${ }^{7}$ J. P. Berge, ${ }^{7}$ J. Berryhill, ${ }^{5}$ S. Bertolucci, ${ }^{9}$ B. Bevensee, ${ }^{26}$ A. Bhatti, ${ }^{31} \mathrm{~K}$. Biery ${ }^{7}$ M. Binkley, ${ }^{7}$ D. Bisello, ${ }^{25}$ R. E. Blair, ${ }^{1}$ C. Blocker, ${ }^{3}$ A. Bodek, ${ }^{30}$ W. Bokhari, ${ }^{19}$ V. Bolognesi, ${ }^{2}$ G. Bolla, ${ }^{29}$ D. Bortoletto, ${ }^{29}$ J. Boudreau, ${ }^{28}$ L. Breccia, ${ }^{2}$ C. Bromberg, ${ }^{21}$ N. Bruner, ${ }^{22}$ E. Buckley-Geer, ${ }^{7}$ H. S. Budd, ${ }^{30}$ K. Burkett, ${ }^{20}$ G. Busetto, ${ }^{25}$ A. ByonWagner, $^{7}$ K. L. Byrum, ${ }^{1}$ J. Cammerata, ${ }^{15}$ C. Campagnari, ${ }^{7}$ M. Campbell, ${ }^{20}$ A. Caner, ${ }^{27}$ W. Carithers, ${ }^{18}$ D. Carlsmith, ${ }^{38}$ A. Castro, ${ }^{25}$ D. Cauz, ${ }^{27}$ Y. Cen,${ }^{30}$ F. Cervelli, ${ }^{27}$ P. S. Chang, ${ }^{33}$ P. T. Chang, ${ }^{33}$ H. Y. Chao, ${ }^{33}$ J. Chapman, ${ }^{20}$ X. T. Cheng, ${ }^{33}$ G. Chiarelli, ${ }^{27}$ T. Chikamatsu, ${ }^{36}$ C. N. Chiou, ${ }^{33}$ L. Christofek, ${ }^{13}$ S. Cihangir, ${ }^{7}$ A. G. Clark, ${ }^{10}$ M. Cobal, ${ }^{27}$ E. Cocca, ${ }^{27}$ M. Contreras, ${ }^{5}$ J. Conway, ${ }^{32}$ J. Cooper ${ }^{7}$ M. Cordelli, ${ }^{9}$ C. Couyoumtzelis, ${ }^{10}$ D. Crane, ${ }^{1}$ D. Cronin-Hennessy, ${ }^{6}$ R. Culbertson, ${ }^{5}$ T. Daniels, ${ }^{19}$ F. DeJongh, ${ }^{7}$ S. Delchamps, ${ }^{7}$ S. Dell'Agnello. ${ }^{27}$ M. Dell'Orso, ${ }^{27}$ R. Demina, ${ }^{7}$ L. Demortier, ${ }^{31}$ M. Deninno, ${ }^{2}$ P. F. Derwent. ${ }^{7}$ T. Devlin, ${ }^{32}$ J. R. Dittmann, ${ }^{6}$ S. Donati, ${ }^{27}$ J. Done, ${ }^{34}$ T. Dorigo, ${ }^{25}$ A. Dunn, ${ }^{20}$ N. Eddy, ${ }^{20} \mathrm{~K}$. Einsweiler, ${ }^{18}$ J. E. Elias, ${ }^{7}$ R. Ely ${ }^{18}$ E. Engels, Jr. ${ }^{28}$ D. Errede. ${ }^{13}$ S. Errede, ${ }^{13}$ Q. Fan,${ }^{30}$ G. Feild,${ }^{39}$ C. Ferretti, ${ }^{2 \pi}$ I. Fiori, ${ }^{2}$ B. Flaugher,${ }^{7}$ G. W. Foster. ${ }^{7}$ M. Franklin, ${ }^{11}$ M. Frautschi ${ }^{35}$ J. Freeman. ${ }^{7}$ J. Friedman, ${ }^{19}$ H. Frisch, ${ }^{5}$ Y. Fukui. ${ }^{17}$ S. Funaki, ${ }^{36}$ S. Galeotti ${ }^{27}$.M. Gallinaro, ${ }^{26}$ O. Ganel ${ }^{35}$ M. Garcia-Sciveres, ${ }^{18}$ A. F. Garfinkel, ${ }^{29}$ C. Gay, ${ }^{11}$ S. Geer, ${ }^{7}$ D. W. Gerdes, ${ }^{15}$ P. Giannetti, ${ }^{27}$ N. Giokaris, ${ }^{31}$ 
P. Giromini, ${ }^{9}$ G. Giusti, ${ }^{2 \pi}$ L. Gladney, ${ }^{26}$ D. Glenzinshi, ${ }^{15}$ M. Gold. ${ }^{22}$ J. Gonzalez. ${ }^{26}$ A. Gordon, ${ }^{11}$ A. T. Goshaw, ${ }^{6}$ Y. Gotra, ${ }^{25}$ K. Goulianos, ${ }^{31}$ H. Grassmann.27 L. Groer ${ }^{32}$ C. Grosso-Pilcher, ${ }^{5}$ G. Guillian, ${ }^{20}$ R. S. Guo, ${ }^{33}$ C. Haber, ${ }^{18}$ E. Hafen,${ }^{19}$ S. R. Hahn, ${ }^{7}$ R. Hamilton, ${ }^{11}$ R. Handler, ${ }^{38}$ R. M. Hans, ${ }^{39}$ F. Happacher,${ }^{9}$ K. Hara ${ }^{36}$ A. D. Hardman, ${ }^{29}$ B. Harral, ${ }^{26}$ R. M. Harris, ${ }^{7}$ S. A. Hauger, ${ }^{6}$ J. Hauser. ${ }^{4}$ C. Hawk, ${ }^{32}$ E. Hayashi, ${ }^{36}$ J. Heinrich, ${ }^{26}$ B. Hinrichsen, ${ }^{14}$ K. D. Hoffman, ${ }^{29}$ M. Hohlmann, ${ }^{5}$ C. Holck, ${ }^{26}$ R. Hollebeek, ${ }^{26}$ L. Holloway, ${ }^{13}$ S. Hong, ${ }^{20}$ G. Houk, ${ }^{26}$ P. Hu, ${ }^{28}$ B. T. Huffman, ${ }^{28}$ R. Hughes, ${ }^{23}$ J. Huston, ${ }^{21}$ J. Huth, ${ }^{11}$ J. Hylen, $\mathrm{H}$. Ikeda, ${ }^{36} \mathrm{M}$. Incagli, ${ }^{27} \mathrm{~J}$. Incandela, ${ }^{7} \mathrm{G}$. Introzzi, ${ }^{27} \mathrm{~J}$. Iwai, ${ }^{36} \mathrm{Y}$. Iwata, ${ }^{12}$ H. Jensen, ${ }^{7}$ U. Joshi, ${ }^{7}$ R. W. Kadel, ${ }^{18}$ E. Kajfasz, ${ }^{25}$ H. Kambara, ${ }^{10}$ T. Kamon, ${ }^{34}$ T. Kaneko, ${ }^{36}$ K. Karr, ${ }^{37}$ H. Kasha, ${ }^{39}$ Y. Kato, ${ }^{24}$ T. A. Keaffaber, ${ }^{29}$ K. Kelley, ${ }^{19}$ R. D. Kennedy, ${ }^{7}$ R. Kephart, ${ }^{7}$ P. Kesten, ${ }^{18}$ D. Kestenbaum, ${ }^{11}$ H. Keutelian, ${ }^{7}$ F. Keyvan, ${ }^{4}$ B. Kharadia, ${ }^{13}$ B. J. Kim,${ }^{30}$ D. H. Kim, ${ }^{7 a}$ H. S. Kim, ${ }^{14}$ S. B. Kim, ${ }^{20}$ S. H. Kim ${ }^{36}$ Y. K. Kim, ${ }^{18}$ L. Kirsch, ${ }^{3}$ P. Koehn, ${ }^{23}$ K. Kondo, ${ }^{36}$ J. Konigsberg, ${ }^{8}$ S. Kopp, ${ }^{5}$ K. Kordas, ${ }^{14}$ A. Korytov, ${ }^{8}$ W. Koska, ${ }^{7}$ E. Kovacs, ${ }^{7 a}$ W. Kowald ${ }^{6}$ M. Krasberg, ${ }^{20}$ J. Kroll, ${ }^{7}$ M. Kruse,${ }^{30}$ T. Kuwabara,${ }^{36}$ S. E. Kuhlmann, ${ }^{1}$ E. Kuns, ${ }^{32}$ A. T. Laasanen, ${ }^{29}$ S. Lami, ${ }^{27}$ S. Lammel, ${ }^{7}$ J. I. Lamoureux, ${ }^{3}$ M. Lancaster, ${ }^{18}$ T. LeCompte, ${ }^{1}$ S. Leone, ${ }^{27}$ J. D. Lewis, ${ }^{7}$ P. Limon, ${ }^{7}$ M. Lindgren, ${ }^{4}$ T. M. Liss, ${ }^{13}$ J. B. Liu, ${ }^{30}$ Y. C. Liu, ${ }^{33}$ N. Lockyer, ${ }^{26}$ O. Long, ${ }^{26}$ C. Loomis, ${ }^{32}$ M. Loreti, ${ }^{25}$ J. Lu, ${ }^{34}$ D. Lucchesi, ${ }^{27}$ P. Lukens, ${ }^{7}$ S. Lusin, ${ }^{38}$ J. Lys, ${ }^{18}$ K. Maeshima, ${ }^{7}$ A. Maghakian, ${ }^{31}$ P. Maksimovic, ${ }^{19}$ M. Mangano, ${ }^{27}$ J. Mansour, ${ }^{21}$ M. Mariotti, ${ }^{25}$ J. P. Marriner, ${ }^{7}$ A. Martin, ${ }^{39}$ J. A. J. Matthews, ${ }^{22}$ R. Mattingly, ${ }^{19}$ P. McIntyre, ${ }^{34}$ P. Melese, ${ }^{31}$ A. Menzione, ${ }^{27}$ E. Meschi, ${ }^{27}$ S. Metzler, ${ }^{26}$ C. Miao, ${ }^{20}$ T. Miao, ${ }^{7}$ G. Michail, ${ }^{11}$ R. Miller ${ }^{21} \mathrm{H}$. Minato, ${ }^{36} \mathrm{~S}$. Miscetti, ${ }^{9}$ M. Mishina, ${ }^{17} \mathrm{H}$. Mitsushio, ${ }^{36}$ T. Miyamoto ${ }^{36}$ S. Miyashita, ${ }^{36} \mathrm{~N}$. Moggi, ${ }^{27}$ Y. Morita, ${ }^{17}$ A. Mukherjee, ${ }^{7}$ T. Muller,${ }^{16}$ P. Murat ${ }^{27}$ H. Nakada, ${ }^{36}$ I. Nakano, ${ }^{36}$ C. Nelson, ${ }^{7}$ D. Neuberger, ${ }^{16}$ C. Newman-Holmes, ${ }^{7}$ CY. P. Ngan, ${ }^{19}$ M. Ninomiya ${ }^{36}$ L. Nodulman, ${ }^{1}$ S. H. Oh ${ }^{6}$ K. E. Ohl ${ }^{39}$ T. Ohmoto, ${ }^{12}$ T. Ohsugi, ${ }^{12}$ R. Oishi, ${ }^{36}$ M. Okabe, ${ }^{36}$ T. Okusawa, ${ }^{24}$ R. Oliveira, ${ }^{26}$ J. Olsen, ${ }^{38}$ C. Pagliarone, ${ }^{27}$ R. Paoletti ${ }^{27}$ V. Papadimitriou, ${ }^{35}$ S. P. Pappas, ${ }^{39}$ N. Parashar, ${ }^{2 \pi}$ S. Park,${ }^{7}$ A. Parri,${ }^{9}$ J. Patrick ${ }^{7}$ G. Pauletta, ${ }^{27}$.M. Paulini, ${ }^{18}$ A. Perazzo,${ }^{27}$ L. Pescara,${ }^{25}$ M. D. Peters, ${ }^{18}$ T. J. Phillips, ${ }^{6}$ G. Piacentino,${ }^{27}$ M. Pillai, ${ }^{30}$ K. T. Pitts, ${ }^{7}$ R. Plunkett, ${ }^{7}$ L. Pondrom, ${ }^{38}$ J. Proudfoot, ${ }^{1}$ F. Ptohos, ${ }^{11}$ G. Punzi, ${ }^{27}$ K. Ragan, ${ }^{14}$ D. Reher ${ }^{18}$ A. Ribon, ${ }^{25}$ F. Rimondi, ${ }^{2}$ L. Ristori, ${ }^{27}$ W. J. Robertson, ${ }^{6}$ T. Rodrigo, ${ }^{27}$ S. Rolli, ${ }^{37}$ J. Romano, ${ }^{5}$ L. Rosenson, ${ }^{19}$ R. Roser, ${ }^{13}$ T. Saab, ${ }^{14}$ W. K. Sakumoto, ${ }^{30}$ D. Saltzberg, ${ }^{5}$ A. Sansoni, ${ }^{9}$ L. Santi, ${ }^{27}$ H. Sato,${ }^{36}$ P. Schlabach, ${ }^{7}$ E. E. Schmidt, ${ }^{7}$ M. P. Schmidt,${ }^{39}$ A. Scribano, ${ }^{27}$ S. Segler, ${ }^{7}$ S. Seidel, ${ }^{22}$ Y. Seiya, ${ }^{36}$ G. Sganos, ${ }^{14}$ M. D. Shapiro, ${ }^{18}$ N. M. Shaw, ${ }^{29}$ Q. Shen, ${ }^{29}$ P. F. Shepard, ${ }^{28}$ M. Shimojima, ${ }^{36}$ M. Shochet, ${ }^{5}$ J. Siegrist, ${ }^{18}$ A. Sill, ${ }^{35}$ P. Sinervo, ${ }^{14}$ P. Singh, ${ }^{28}$ J. Skarha ${ }^{15}$ K. Sliwa, ${ }^{37}$ F. D. Snider ${ }^{15}$ T. Song, ${ }^{20}$ J. Spalding, ${ }^{7}$ T. Speer, ${ }^{10}$ P. Sphicas, ${ }^{19}$ F. Spinella, ${ }^{27}$ M. Spiropulu, ${ }^{11}$ L. Spiegel, ${ }^{7}$ L. Stanco, ${ }^{25}$ J. Steele, ${ }^{38}$ A. Stefanini, ${ }^{27}$ K. Strahl, ${ }^{1+}$ J. Strait. ${ }^{7}$ R. Ströhmer, ${ }^{7 a}$ D. Stuart, ${ }^{7}$ G. Sullivan, ${ }^{5}$ K. Sumorok, ${ }^{19}$ J. Suzuki, ${ }^{36}$ T. Takada ${ }^{36}$ T. Takahashi, ${ }^{24} \mathrm{~T}$. Takano, ${ }^{36} \mathrm{~K}$. Takikawa, ${ }^{36} \mathrm{~N}$. Tamura, ${ }^{12} \mathrm{~B}$. Tannenbaum, ${ }^{22}$ F. Tartarelli, ${ }^{27}$ W. Taylor, ${ }^{14}$ P. K. Teng, ${ }^{33}$ Y. Teramoto, ${ }^{24}$ S. Tether, ${ }^{19}$ D. Theriot, ${ }^{7}$ 
T. L. Thomas. ${ }^{22}$ R. Thun, ${ }^{20}$ R. Thurman-Keup, ${ }^{1}$ M. Timko ${ }^{37}$ P. Tipton. ${ }^{30}$ A. Titor ${ }^{31}$ S. Tkaczyk, ${ }^{7}$ D. Toback, ${ }^{7}$ K. Tollefson, ${ }^{30}$ A. Tollestrup, ${ }^{7}$ H. Toyoda, ${ }^{24}$ W. Trischuk..$^{14}$ J. F. de Troconiz, ${ }^{11} \mathrm{~S}$. Truitt, ${ }^{20} \mathrm{~J}$. Tseng, ${ }^{19} \mathrm{~N}$. Turini, ${ }^{2 \mathrm{~T}} \mathrm{~T}$. Uchida ${ }^{36} \mathrm{~N}$. Lemura ${ }^{36}$ F. Ukegawa, ${ }^{26}$ G. Unal, ${ }^{26}$ J. Valls, ${ }^{7 a}$ S. C. van den Brink, ${ }^{28}$ S. Vejcik, III, ${ }^{20}$ G. Velev, ${ }^{27}$ R. Vidal, ${ }^{7}$ R. Vilar, ${ }^{7 a}$ M. Vondracek, ${ }^{13}$ D. Vucinic, ${ }^{19}$ R. G. Wagner,${ }^{1}$ R. L. Wagner,${ }^{7}$ J. Wahl, ${ }^{5}$ N. B. Wallace, ${ }^{27}$ A. M. Walsh, ${ }^{32}$ C. Wang, ${ }^{6}$ C. H. Wang, ${ }^{33}$ J. Wang, ${ }^{5}$ M. J. Wang, ${ }^{33}$ Q. F. Wang, ${ }^{31}$ A. Warburton, ${ }^{14}$ T. Watts, ${ }^{32}$ R. Webb, ${ }^{34}$ C. Wei. ${ }^{6}$ H. Wenzel, ${ }^{16}$ W. C. Wester, III, ${ }^{7}$ A. B. Wicklund, ${ }^{1}$ E. Wicklund, ${ }^{7}$ R. Wilkinson, ${ }^{26}$ H. H. Williams, ${ }^{26}$ P. Wilson, ${ }^{5}$ B. L. Winer, ${ }^{23}$ D. Winn, ${ }^{20}$ D. Wolinski, ${ }^{20}$ J. Wolinski. ${ }^{21}$ S. Worm, ${ }^{22} \mathrm{X}$. Wu, ${ }^{10} \mathrm{~J}$. Wyss, ${ }^{25}$ A. Yagil, ${ }^{7} \mathrm{~W}$. Yao, ${ }^{18} \mathrm{~K}$. Yasuoka, ${ }^{36} \mathrm{Y}$. Ye, ${ }^{14}$ G. P. Yeh, ${ }^{7}$ P. Yeh, ${ }^{33}$ M. Yin ${ }^{6}$ J. Yoh, ${ }^{7}$ C. Yosef, ${ }^{21}$ T. Yoshida, ${ }^{24}$ D. Yovanovitch, ${ }^{7}$ I. Yu, ${ }^{7}$ L. Yu, ${ }^{22}$ J. C. Yun, ${ }^{7}$ A. Zanetti, ${ }^{27}$ F. Zetti, ${ }^{27}$ L. Zhang, ${ }^{38}$ W. Zhang, ${ }^{26}$ and S. Zucchelli ${ }^{2}$

\section{(CDF Collaboration)}

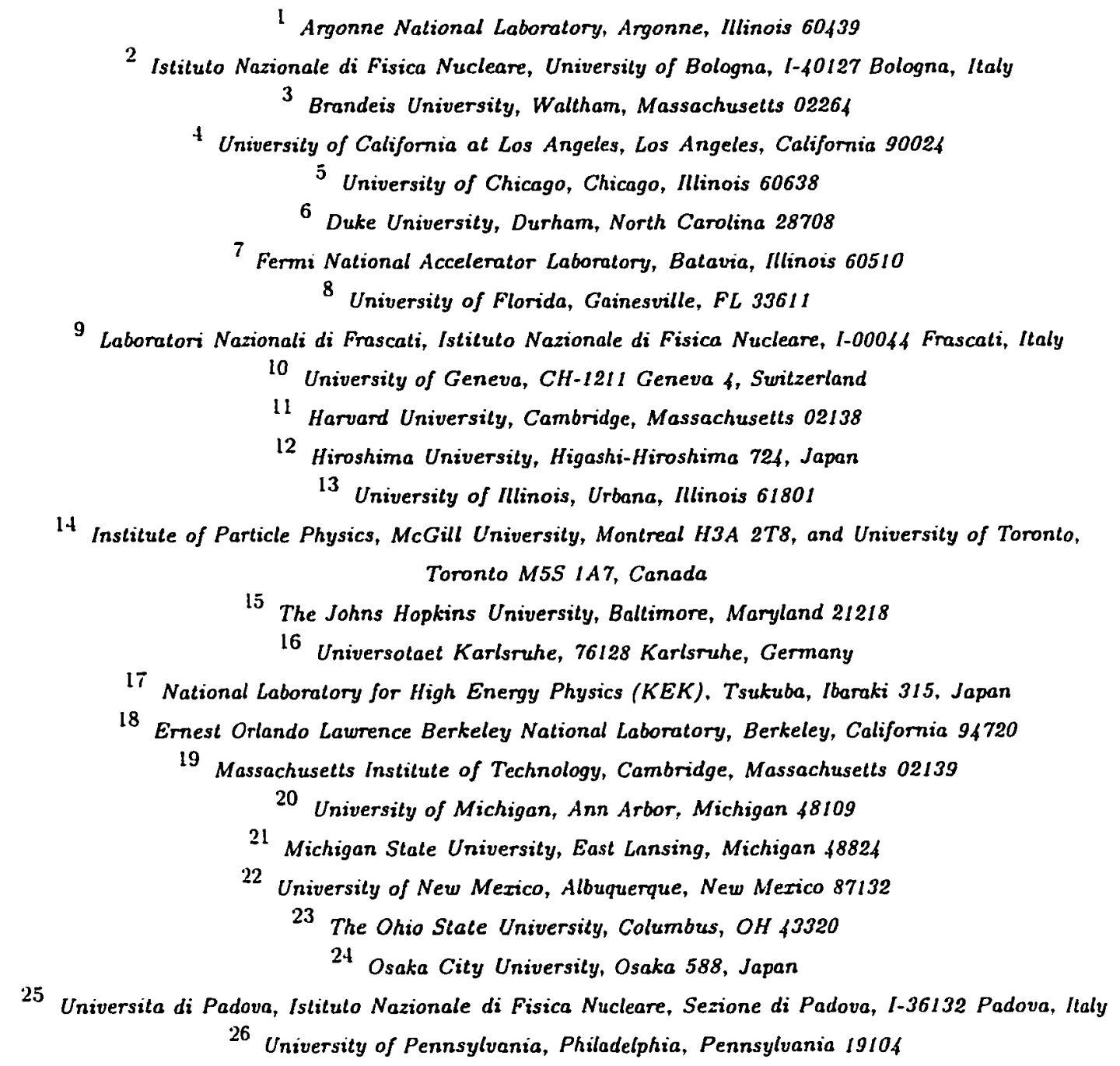


30 University of Rochester, Rochester, New York 14628

31 Rockefeller University, New York, New York 10021

32 Rutgers University, Piscatoway, New Jersey 08854

33 Academia Sinica, Taipei, Taiwan 11530, Republic of China

34 Texas A\&M University, College Station, Texas 77843

35 Texas Tech Universily, Lubbock, Texas 79409

36 University of Tsukuba, Tsukuba, Ibaraki 315, Japan

37 Tufts University, Medford, Massachusetts 02155

38 University of Wisconsin, Madison, Wisconsin 53806

39 Yale University, New Haven, Connecticut 06511 


\section{References}

[1] S. Weinberg, A Model of Leptons, Phys. Rev. Lett. 19, 1264 (1967).

[2] A. Salam, in Elementary Particle Theory: Relativistic Groups and Analyticity (Nobel Symposium No. 8), edited by N. Svartholm (Almqvist and Wiksell, Stockholm, 1968) p. 367.

[3] UA1 Collaboration, G. Arnison et al.., Phys. Lett. B 122B 103 (1983); Phys. Lett. 126B 398 (1983).

[4] UA2 Collaboration, M. Banner et al., Phys. Lett. B 122B 476 (1983).

[5] R. M. Barnett et al., Phys. Rev. D 54, 1 (1996).

[6] N. Cabibbo, Phys. Rev. Lett. 10, 531 (1963); .M. Kobayashi and T. Maskawa, Prog. Theor. Phys. 49, 652 (1972).

[7] UA1 Collaboration, G. Arnison et al., Phys. Lett. B 126B 398 (1983).

[8] LA2 Collaboration, P. Bagnaia et al., Phys. Lett. B 129B 130 (1983).

[9] S. W. Herb, et al., Phys. Rev. Lett. 39, 252 (1977).

[10] CDF Collaboration, F. Abe et al., Phys. Rev. Lett. 74, 2626 (1995).

[11] D0 Collaboration, S. Abachi et al., Phys. Rev. Lett. 74, 2626 (1995).

[12] J. Lys, Pub. Proceedings 28th International Conference on High Energy Physics (ICHEP'96), Warsaw, Poland, July 25-31, 1996. FERMILAB-CONF-96/409-E. 
[13] D0 Collaboration. S. Abachi et al., FER.MILAB-Pub-9T/059-E. to be submitted to Phys. Rev. Lett.

[14] I. Bigi et al., Phys. Lett. B 181. 157.

[15] CDF Collaboration, F. Abe et al., Phys. Rev. Lett. in preparation.

[16] A. Castro, Published Proceedings 32nd Rencontres de Moriond: QCD and HighEnergy Hadronic Interactions, Les Arcs, France, March 22-29, 1997.

[17] E. Laenen, J. Smith, and W. L. van Neerven, Phys. Lett. B 321254 (1994).

[18] E. L. Berger and H. Contopanagos, Phys. Lett. B 361115 (1995).

[19] S. Catani et al., CERN Preprint CERN-TH/96-21.

[20] "Future Electroweak Physics at the Fermilab Tevatron", edited by D. Amidei and R. Brock, FERMILAB-Pub-96/046 (unpublished).

[21] J. Pumplin, Phys. Rev. D 44, 2025 (1991).

[22] CDF Collaboration, F. Abe et al., Nucl. Instrum. Methods Phys. Res. A 271. 387 (1988); FERMILAB-Pub-94/024-E, 1994 (unpublished).

[23] D. Amidei et al., Nucl. Instrum. Methods Phys. Res. A 350, 73 (1994).

[24] S. Cihangir et al., Nucl. Instrum. Methods Phys. Res., Sect. A 360, 137 (1995).

[25] CDF Collaboration, F. Abe et al., Phys. Rev. D 45, 1448 (1992); CDF Collaboration, F. Abe et al., , Phys. Rev. D 47, 4857 (1993). 
[26] CDF Collaboration. F. Abe et al., Phys. Rev. D 48. 3939 (1993).

[27] R. Hughes, T. Liss, CDF internal note 2992.

[28] T. Liss, R. Roser, A. Martin, CDF internal note 3931.

[29] G. Houk, Ph. D. thesis, University of Pennsylvania.

[30] D. Kestenbaum, Ph. D. thesis, Harvard University.

[31] D. Buskulic et al., Phys. Lett. B313, 535 (1993).

[32] G. Marchesini and B. R. Webber, Nucl. Phys. B310, 461 (1994)

G. Marchesini et al., Comput. Phys. Comm. 67, 456 (1992).

[33] F.A.Berends, W.T.Giele, H.Kuif, B.Tausk, Nucl. Phys. B 357, 32 (1991). W.Giele, Ph. D. thesis, Leiden.

[34] F. Paige and S. D. Protopopescu, BNL Report No. 38034, 1986 (unpublished).

[35] J. Lys, A. B. Galtieri, CDF internal note 4075.

[36] G. Unal, CDF internal note 3389.

[37] T. Steltzer and S. Willenbrock, Phys. Lett. B 357 125-130, 1995.

[38] CDF Collaboration, F. Abe et al., Phys. Rev. Lett. 77, 438 (1996).

[39] S. Aota, Ph. D. thesis, U. of Tsukuba.

[40] T. Sjöstrand, Comp. Phys. Comm. 82, 74 (1994). 
[41] J. Alitti, et al., Z. Phys. C49, 17 (1991).

[42] S. Kuhlmann, private communication.

[43] J. Pumplin, Phys. Rev. D45, 806 (1992).

[44] K. Hagiwara, J. Woodside, and D. Zeppenfeld, Phys. Rev, D 41, 2113 (1990).

[45] J. Ohnemus, Phys. Rev. D 44, 1403 (1991); J. Ohnemus, Phys. Rev. D 44 (1991) 3477; J. Ohnemus and J. F. Owens, Phys. Rev. D 43, 3626 (1991).

[46] CDF Collaboration, F. Abe et al., Phys. Rev. Lett. 78, 4536 (1997).

[47] CDF Collaboration, F. Abe et al., Phys. Rev. Lett. 75, 1017 (1995).

[48] D0 Collaboration, S. Abachi et al., Phys. Rev. Lett. 77, 3303 (1996).

[49] V. Barger and R. Phillips, Collider Physics (Addison-Wesley, New York, 1987).

[50] E. Eichten, I. Hinchliffe, K. Lane, and C. Quigg, Rev. Mod. Phys. 56 (1984) 579: 58, (1986) 1065(E).

[51] N. Eddy et al., CDF internal note 3036.

[52] CDF Collaboration, F. Abe et al., , Observation of Hadronic $W$ decays in $t \bar{t}$ events with the Collider Detector at Fermilab", to be submitted to Phys. Rev. Lett.

[53] S. Kim, S. Kuhlmann, W. M. Yao, "Improvement of Signal Significance in $W H \rightarrow \ell \nu b \bar{b}$ search at TeV33", Pub. Proceedings 1996 DPF/DPB Summer 
Study, "New Directions for High Energy Physics".

[54] L. Clavelli and I. Terekhov, "Scaling Violations through Squark and Light Gluino Production", preprint hep-ph/9702301.

[55] The CDF II Detector Technical Design Report, FERMILAB-Pub-96/390-E (unpublished).

[56] TeV2000 Report, Top Physics at the Tevatron, edited by D. Amidei and R. Brock, FERMILAB-Pub-96/082 (unpublished). 


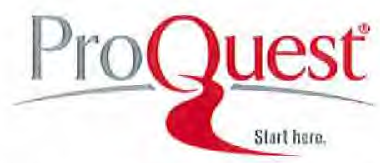

2017 ProQuest Distribution Agreement

This Agreement is between the author (Author) and ProQuest LLC, through its Proquest Dissertation Distribution business (formerly Proquest/UMI). Under this Agreement, Author grants ProQuest certain rights to preserve, archive and distribute the dissertation or thesis (the Work), abstract, and index terms provided by Author to Proquest.

\section{Section I. License for Inclusion of the Work in ProQuest Publishing Program}

Grant of Rights. Author hereby grants to ProQuest the non-exclusive, worldwide right to reproduce, distribute, display and transmit the Work (in whole or in part) in such tangible and electronic formats as may be in existence now or developed in the future. Author further grants to PraQuest the right to include the abstract, bibliography and other metadata in the ProQuest Dissertations \& Theses database (PQDT) and in ProQuest Dissertation Abstracts International and any successor or related index and/or finding products or services.

Proquest Publishing Program - Election and Elements. The rights granted above shall be exercised according to the publishing option selected by Author in Section III, Author Options, and subject to the following additional Publishing Program requirements:

- Distribution of the Work. Except as restricted by Author in the publishing option selected, the rights granted by Author automatically include (1) the right to allow sale and distribution of the Work, in whole or in part, by ProQuest and its sales agents and distributors and (2) the right to make the Abstract, bibliographic data and any meta data associated with the Work available to search engines and harvesters.

- Restrictions. ProQuest will use commercially reasonable efforts to restrict the distribution of the Work as provided under the publishing option selected by Author or as later elected by Author through direct contact with ProQuest. Such election is subject to Author's Degree Granting Institution Directives. With respect to restrictions requested after submission of the Work, Author acknowledges that ProQuest cannot recall or amend previously distributed versions of the Work.

- Removal of Work from the Program. ProQuest may elect not to distribute the Work if it believes that all necessary rights of third parties have not been secured. Refer to the website http://Www.proquest.com/products_umi/dissertations/ for information about copyright and your dissertation or thesis. If Author's degree is rescinded, and/or the degree-granting institution so directs, ProQuest will expunge the Work from its publishing program in accordance with its then current publishing policies.

- Degree Granting Institution Directives. Author is solely responsible for any conflict between policies and directions of Author's degree-granting institution, Author's choice of publishing model, and/or any restriction Author places on the use of the Work. For the avoidance of doubt, ProQuest is not responsible for access to the Work that is provided by Author's degree-granting institution through its library or institutional repository. Author must work directly with Author's degree granting institution to ensure application of any restrictions to access relating to the Work by Author's degree granting institution.

Delivery of the Work. Author shall provide to ProQuest the Work and all necessary supporting documents during the online submission process, according to the Instructions accompanying this agreement.

Rights Verification. Author represents and warrants that Author is the copyright holder of the Work and has obtained all necessary rights to permit ProQuest to reproduce and distribute third party materials contained in any part of the Work, including all necessary licenses for any non-public, third party software necessary to access, display, and run or print the Work. Author is solely responsible and will indernnify ProQuest for any third party claims related to the Work as submitted for publication.

Open Access Publishing Plus

Q I want the broadest possible dissemination of my work, and I want to provide free global access to the electronic copy of my workvia the internet.

4 I understand that I will not be eligible to receive royalties.

I want major search engines (e.g, Google, Yahoo) to discover my work. Learn more: http://www.proquest.com/enus/products/dissertations/google.shtml

$$
\begin{aligned}
& \text { 1) Yes } \\
& \square \text { No }
\end{aligned}
$$

Acknowledgment: I have read, unders/gnd and agree fo this Proquest Publishing Agreement, including all rights and restrictions included within the publishing option chosen by he as hidicated ab bve.

REQUIRED Author's signature Date $7.20 \cdot 20 \mathrm{i} 7$

(Print Name) Richord $P$ wi lanicin m


This page must accompany your manuscript and the rest of the submission materials

Dissertation/Master's Thesis Submission Form Please print clearly in block letters

Personal Information

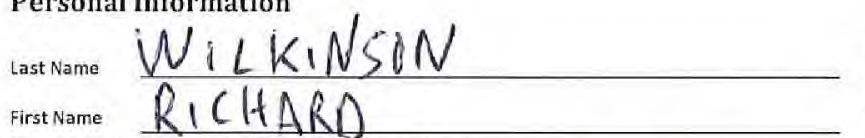

Degree \& Dissertation Information

Title of Dissertation/ Thesis

Institution conferring

degree

HADRONIC DECAYS OF
U. Of PENNSYLVANIA

College, School, or Division

Department or Program

Advisor/Committee Chair

Committee Member

Committee Member
ARTS \& SCIENKES

PHYSICS

PROF. ROBERT HOLLEBEEK

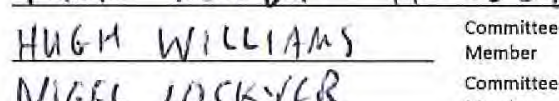

Please check type of manuscript:

_ M (Master's Thesis)

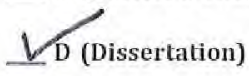

Middle Name or DALL

Country (ies) of 15

Citizenship
Bosons Degree awarded
(abbreviate; e.g. Ph.D.) h. 0 .

Year degree awarded $\quad 1997$ $\begin{array}{ll}\substack{\text { Year manuscript } \\ \text { completed }} & 0,177\end{array}$ 
Primary Subject Category: Enter the 4-digit code and category name from the Subject Category Guide that most closely describes the area of your research.

You may suggest two additional subject categories that may aid in the discovery of your work in our digital database. cote 0798 category ARTICLE PAYSICS

Provide up to 6 keywords or short phrases for citation indices, library cataloging, and database searching.

$\frac{C D F}{W} \frac{T O P}{\text { TADRONiC }}$
Current Con

Street Address

$$
\text { Please provide your postal address if you are interested in receiving royalties on sales of your thesis. }
$$

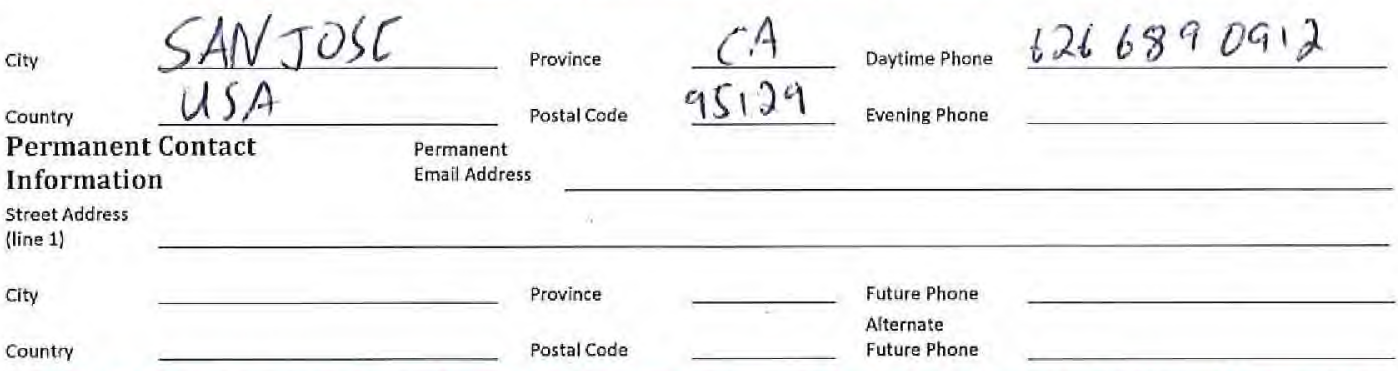

THIS PAGE MUST ACCOMPANY YOUR MANUSCRIPT AND THE REST OF YOUR SUBMISSION MATERIALS

Subject Categories

The ProQuest Dissertations \& Theses (PQDT) database and the ProQuest citation indices are arranged by subject categories. Please select the one category below that best describes your field of research or creative work. You may add one or two additional categories on your submission form that will also be associated with your work as secondary subjects.

\section{Arts, Business, Education, Humanities, and Social Sciences}

\section{AREA, ETHNIC, AND GENDER}

\section{STUDIES}

African American studies $\quad 0296$

African studies

American studies

Asian American studies

Asian studies

Baltic studies

Black studies

Canadian studies

Caribbean studies

Classical studies

East European studies

Ethnic studies

European studies

French Canadian culture

Gender studies

GLBT studies

Hispanic American studies
Holocaust studies

Islamic culture

Judaic studies

Latin American studies

Middle Eastern studies

Native American studies

Near Eastern studies

North African studies

Pacific Rim studies

Regional studies

Scandinavian studies

Slavic studies

South African studies

South Asian studies

Sub Saharan Africa studies

Women's studies
0507

0512

0751

0550

0555

0559
0560

0561

0604

0613

0614

0654

0638

0639

\begin{tabular}{|c|c|}
\hline \multicolumn{2}{|l|}{ BUSINESS } \\
\hline Accounting & 0272 \\
\hline Arts management & 0424 \\
\hline Banking & 0770 \\
\hline Business & 0310 \\
\hline Entrepreneurship & 0429 \\
\hline Finance & 0508 \\
\hline Management & 0454 \\
\hline Marketing & 0338 \\
\hline Sports management & 0430 \\
\hline COMMUNICATIONS & \\
\hline INFORMATION SCI & \\
\hline Communication & 0459 \\
\hline Information science & 0723 \\
\hline Joumalism & 0391 \\
\hline Library science & 0399 \\
\hline Mass communication & 0708 \\
\hline Technical communication & 0643 \\
\hline
\end{tabular}

